\title{
A p-adic Maass-Shimura operator on Mumford curves
}

\author{
Matteo Longo ${ }^{1}$
}

Received: 18 May 2021 / Accepted: 26 November 2021 / Published online: 27 February 2022

(c) The Author(s) 2022

\section{Abstract}

We study a $p$-adic Maass-Shimura operator in the context of Mumford curves defined by [15]. We prove that this operator arises from a splitting of the Hodge filtration, thus answering a question in [15]. We also study the relation of this operator with generalized Heegner cycles, in the spirit of $[1,4,19,28]$.

\section{Résumé}

Nous étudions un opérateur de Maass-Shimura $p$-adique pour les courbes de Mumford défini par [15]. Nous montrons que cet opérateur peut être défini en terme d'une scission de la filtration de Hodge, répondant à une question posée dans [15]. Nous étudions aussi la relation de cet opérateur avec les cycles de Heegner généralisés, comme dans [1, 4, 19, 28].

Keywords $p$-adic uniformisation $\cdot$ Shimura curves $\cdot$ Maass-Shimura operators

Mathematics Subject Classification 11F03 $\cdot$ 14F40 $\cdot 11 \mathrm{R} 52$

\section{Introduction}

The main purpose of this paper is to study in the context of Mumford curves a $p$-adic variant of the Maass-Shimura operator, and relate it to generalized Heegner cycles.

The real analytic Maass-Shimura operator is defined by the formula

$$
\delta_{k}(f)(z)=\frac{1}{2 \pi i}\left(\frac{\partial}{\partial z}+\frac{k}{z-\bar{z}}\right) f(z)
$$

where $z$ is a variable in the complex upper half plane $\mathcal{H}, f$ is a real analytic modular form of weight $k$, and $z \mapsto \bar{z}$ denotes the complex conjugation; here $\delta_{k}(f)$ is a real analytic modular form of weight $k+2$. The relevance of this operator arises in studying algebraicity properties of Eisenstein series and $L$-functions: see Shimura [42], Hida [20, Chapter 10]. One of the

To Bernadette Perrin-Riou on the occasion of her 65th birthday.

Matteo Longo

mlongo@math.unipd.it

1 Dipartimento di Matematica Tullio Levi-Civita, Università degli Studi di Padova, Via Trieste 63, 35121 Padua, Italy 
main results in [42] is the following. Let

$$
\delta_{k}^{r}=\delta_{k+2(r-1)} \circ \delta_{k+2(r-2)} \circ \cdots \circ \delta_{k}
$$

for any $r \geq 1$, and let $K$ be an imaginary quadratic field. Then there exists $\Omega_{K} \in \mathbb{C}^{\times}$such that for every modular form $f$ of weight $k$ with algebraic Fourier coefficients, and for every CM point $z \in K \cap \mathcal{H}$, we have

$$
\frac{\delta_{k}^{r}(f)(z)}{\Omega_{K}^{(k+2 r)}} \in \overline{\mathbb{Q}} .
$$

Katz described in [25] the Maass-Shimura operator in more abstract terms by means of the Gauss-Manin connection (see also [27]). Let $N \geq 1$ be an integer, $X_{1}(N)$ the modular curve of level $\Gamma_{1}(N)$ over $\mathbb{Q}$, and let $\pi: \mathcal{E} \rightarrow X_{1}(N)$ be the universal elliptic curve. Consider the relative de Rham cohomology sheaf

$$
\mathcal{L}_{1}=\mathbb{R}^{1} \pi_{*}\left(0 \rightarrow \mathcal{O}_{\mathcal{E}} \rightarrow \Omega_{\mathcal{E} / X_{1}(N)}^{1}\right)
$$

on $X_{1}(N)$, and define $\mathcal{L}_{r}=\operatorname{Sym}^{r}\left(\mathcal{L}_{1}\right)$. Let $\underline{\omega}=\pi_{*}\left(\Omega_{\mathcal{E} / X_{1}(N)}^{1}\right)$. The sheaf $\underline{\omega}$ is invertible and we have the Hodge filtration

$$
0 \rightarrow \underline{\omega} \rightarrow \mathcal{L}_{1} \rightarrow \underline{\omega}^{-1} \rightarrow 0 .
$$

Considering the associated real analytic sheaves, which we denote by a superscript ${ }^{\text {ran }}$, the Hodge exact sequence of real analytic sheaves associated with (3) admits indeed a splitting

$$
\Psi_{\infty}: \mathcal{L}_{1}^{\mathrm{ran}} \stackrel{\sim}{\longrightarrow} \underline{\omega}^{\mathrm{ran}} \oplus \underline{\bar{\omega}}^{\mathrm{ran}},
$$

where $\underline{\bar{\omega}}_{\text {ran }}$ is obtained from $\underline{\omega}$ by applying the complex conjugation. The Maass-Shimura operator

$$
\Theta_{\infty, r}:\left(\underline{\omega}^{\mathrm{ran}}\right)^{\otimes r} \longrightarrow\left(\underline{\omega}^{\mathrm{ran}}\right)^{\otimes(r+2)}
$$

can then be defined combining the splitting (4) with the Gauss-Manin connection and the Kodaira-Spencer map. For details, the reader is referred to [26, Sect. 1.8] and [4, Sect. 1.2]; for the case of Siegel modular forms, see [17, Sect. 4] while for the case of Shimura curves see [19, Sect. 3], [37, Sect. 2].

As hinted from the above discussion, Katz description of the Maass-Shimura operator rests on the fact that the real analytic Hodge sequence (3) splits. In [26, Sect. 1.11], Katz introduces a $p$-adic analogue of this splitting. Suppose that $p \nmid N$ is a prime number, and let $X_{1}^{\text {ord }}(N)$ denote the ordinary locus of the modular curve, viewed as a rigid analytic scheme over $\mathbb{Q}_{p}$. Let $\mathcal{F}^{\text {rig }}$ be the rigid analytic sheaf associated with a sheaf $\mathcal{F}$ on $X_{1}(N)$. Then $\mathcal{L}_{1}^{\text {rig }}$ splits over $X_{1}^{\text {ord }}(N)$ as the direct sum

$$
\Psi_{p}: \mathcal{L}_{1}^{\text {rig }} \simeq \underline{\omega}^{\text {rig }} \oplus \mathcal{L}_{1}^{\text {Frob }}
$$

(where $\mathcal{L}_{1}^{\text {Frob }}$ has the property that the Frobenius endomorphism acts invertibly on this sheaf). This allows to define a differential operator $\Theta_{p, r}$, which can be seen as a $p$-adic analogue of the Maass-Shimura operator; this operator can be described in terms of Atkin-Serre derivative. At CM points the splittings $\Psi_{\infty}$ and $\Psi_{p}$ coincide, and therefore one deduces rationality results for the values of $\Theta_{p, r}$ at CM points from (2). For details, see [4, Proposition 1.12]. The $p$-adic Maass-Shimura operator is then used in $[4,26]$ to construct $p$-adic $L$-functions and study their properties. 
We now fix an integer $N$, a prime $p \nmid N$, and a quadratic imaginary field in which $p$ is inert. In this context, Kritz introduced in [28] for modular forms of level $N$ a new $p$-adic Maass-Shimura operator by using perfectoid techniques, and defined $p$-adic $L$-functions by means of this operator, thus removing the crucial assumption that $p$ is split in $K$, but keeping the assumption that $p$ is a prime of good reduction for the modular curve. Moreover, Andreatta-Iovita [1] introduced still another $p$-adic Maass-Shimura operator in [1], and obtained results analogous to those in [4], thus extending their work to the non-split case.

On the other hand, Franc in his thesis [15] proposed still another $p$-adic Maass-Shimura operator for primes $p$ which are inert in $K$, in the following context. Let $N \geq 1$ be an integer, $K / \mathbb{Q}$ a quadratic imaginary field, $p \nmid N$ a prime number, $p \geq 5$, which is inert in $K$, and let $N p=N^{+} \cdot N^{-} p$ be a factorization of $N p$ into coprime integers such that $N^{+}$is divisible only by primes which are split in $K$, and $N^{-} p$ is a square-free product of an even number of prime factors which are inert in $K$. Let $\mathcal{B}$ be the indefinite quaternion algebra of discriminant $N^{-} p$, $\mathcal{R}$ an Eichler order of $\mathcal{B}$ of level $N^{+}$, and $X$ the Shimura curve attached to $(\mathcal{B}, \mathcal{R})$. The rigid analytic curve $X^{\text {rig }}$ over $\mathbb{Q}_{p}$ is then a Mumford curve, namely $X\left(\mathbb{C}_{p}\right)$ is isomorphic to the rigid analytic quotient of the $p$-adic upper half plane $\mathcal{H}_{p}\left(\mathbb{Q}_{p}\right)=\mathbb{C}_{p}-\mathbb{Q}_{p}$ by an arithmetic subgroup of $\mathrm{SL}_{2}\left(\mathbb{Q}_{p}\right)$. Franc defines in this context a $p$-adic Maass-Shimura operator $\delta_{p, k}$ by mimicking the definition (1), formally replacing the variable $z \in \mathcal{H}$ with the $p$-adic variable $z \in \mathcal{H}_{p}\left(\hat{\mathbb{Q}}_{p}^{\text {unr }}\right)=\hat{\mathbb{Q}}_{p}^{\text {unr }}-\mathbb{Q}_{p}$, and replacing the complex conjugation with the Frobenius map (here $\hat{\mathbb{Q}}_{p}^{\text {unr }}$ is the completion of the maximal unramified extension of $\mathbb{Q}_{p}$ ). Following the arguments of [42], Franc proves a statement analogous to (2) (see [15, Theorem 5.1.5]).

In [15, Sect. 6.1.3], Franc asks for a construction of his $p$-adic Maass-Shimura operator by means of a (non-rigid analytic) splitting $\Psi_{p}$ of the Hodge filtration, similar to what happens over $X_{1}(N)$ (in the real analytic case, [42]) and $X_{1}^{\text {ord }}(N)$ (in the $p$-adic rigid analytic case, [26]). The first result of this paper is to provide such a splitting $\Psi_{p}$, and define the associated $p$-adic Maass-Shimura operator. In particular, we show that our splitting $\Psi_{p}$ coincides at CM points with the Hodge splitting $\Psi_{\infty}$, and therefore, as in [26], we reprove the main results of [15] by the comparison of the two Shimura-Mass operators. We also derive a relation between our $p$-adic Maass-Shimura operator and generalized Heegner cycles in the context of Mumford curves, which can be viewed as an analogue of [4, Proposition 3.24]. In the remaining part of the introduction we describe more precisely the results of this paper.

Instead of the curve $X$ attached to the Eichler order $\mathcal{R}$, we follow [19] and consider a covering $\mathcal{C} \rightarrow X$ where $\mathcal{C}$ is a geometrically connected curve defined over $\mathbb{Q}$ corresponding to a $V_{1}\left(N^{+}\right)$-level structure. The curve $\mathcal{C}$ is the solution of a moduli problem, and we have a universal false elliptic curve $\pi: \mathcal{A} \rightarrow \mathcal{C}$ (see Sect. 2.2). Following [18, 19, 37], we define a quaternionic projector $e$, acting on the relative de Rham cohomology of $\pi: \mathcal{A} \rightarrow \mathcal{C}$, and define the sheaf

$$
\mathcal{L}_{1}=e \cdot \mathcal{H}_{\mathrm{dR}}^{1}(\mathcal{A} / \mathcal{C})
$$

and the line bundle

$$
\underline{\omega}=e \cdot \pi_{*}\left(\Omega_{\mathcal{A} / \mathcal{C}}^{1}\right) .
$$

We have a corresponding Hodge filtration

$$
0 \longrightarrow \underline{\omega} \longrightarrow \mathcal{L}_{1} \longrightarrow \underline{\omega}^{-1} \longrightarrow 0 .
$$

The rigid analytic curve $\mathcal{C}^{\text {rig }}$ associated with $\mathcal{C}$ admits a $p$-adic uniformization

$$
\mathcal{C}^{\mathrm{rig}}\left(\mathbb{C}_{p}\right) \simeq \Gamma_{p} \backslash \mathcal{H}_{p}\left(\mathbb{C}_{p}\right)
$$


for a suitable subgroup $\Gamma_{p} \subseteq \mathrm{SL}_{2}\left(\mathbb{Q}_{p}\right)$. Modular forms on $\mathcal{C}^{\text {rig }}$ are then $\Gamma_{p}$-invariant sections of $\mathcal{H}_{p}$, and therefore, to define a $p$-adic Maass-Shimura operator on $\mathcal{C}$ one is naturally led to consider the analogous problem for the covering $\mathcal{H}_{p}$ of $\mathcal{C}^{\text {rig }}$.

Let $\mathcal{C}^{0}$ denote the $\mathbb{C}_{p}$-vector space of continuous (for the standard $p$-adic topology on both spaces) $\mathbb{C}_{p}$-valued functions on $\mathcal{H}_{p}\left(\hat{\mathbb{Q}}_{p}^{\text {unr }}\right)$, and let $\mathcal{A}$ denote the $\hat{\mathbb{Q}}_{p}^{\text {unr }}$-vector space of rigid analytic global sections of $\mathcal{H}_{p}\left(\hat{\mathbb{Q}}_{p}^{\text {unr }}\right)$. We have a map of $\hat{\mathbb{Q}}_{p}^{\text {unr }}$-vector spaces $r: \mathcal{A} \rightarrow \mathcal{C}^{0}$ and, following [15], we denote $\mathcal{A}^{*}$ the image of the morphism of $\mathcal{A}$-algebras $\mathcal{A}[X, Y] \rightarrow \mathcal{C}^{0}$ defined by sending $X$ to the function $z \mapsto 1 /(z-\sigma(z))$ and $Y$ to the function $z \mapsto \sigma(z)$, where $\sigma: \hat{\mathbb{Q}}_{p}^{\mathrm{unr}} \rightarrow \hat{\mathbb{Q}}_{p}^{\mathrm{unr}}$ is the Frobenius automorphism (note that the function $z \mapsto z-\sigma(z)$ is invertible on $\mathcal{H}_{p}\left(\hat{\mathbb{Q}}_{p}^{\text {unr }}\right)$ ). Denote $\hat{\mathcal{H}}_{p}$ the formal $\mathbb{Z}_{p}$-scheme whose generic fiber is $\mathcal{H}_{p}$, let $\hat{\mathcal{H}}_{p}^{\text {unr }}$ be its base change to $\hat{\mathbb{Q}}_{p}^{\text {unr }}$ and let $\mathcal{G} \rightarrow \hat{\mathcal{H}}_{p}^{\text {unr }}$ be the universal special formal module with quaternionic multiplication. Denote $\underline{\omega}_{\mathcal{G}}=e_{\mathcal{G}}^{*}\left(\Omega_{\mathcal{G} / \hat{\mathcal{H}}_{p}^{\text {unr }}}^{1}\right)$, where $e_{\mathcal{G}}: \hat{\mathcal{H}}_{p}^{\text {unr }} \rightarrow \mathcal{G}$ is the zero-section, and let $\mathcal{L} i e_{\mathcal{G}^{\vee}}$ be the Lie algebra of the Cartier dual $\mathcal{G}^{\vee}$ of $\mathcal{G}$. Then $\underline{\omega}_{\mathcal{G}}$ and $\mathcal{L i}_{\mathcal{G}^{\vee}}$ are locally free $\mathcal{O}_{\hat{\mathcal{H}}_{p}^{\text {unr }}}$-modules, dual to each other and we have the Hodge-Tate exact sequence of $\mathcal{O}_{\hat{\mathcal{H}}_{p}^{\text {unr }}}$-modules

$$
0 \longrightarrow \underline{\omega}_{\mathcal{G}} \longrightarrow \mathcal{H}_{\mathrm{dR}}^{1}\left(\mathcal{G} / \hat{\mathcal{H}}_{p}^{\mathrm{unr}}\right) \longrightarrow \mathcal{L}_{i e_{\mathcal{G}}} \longrightarrow 0 .
$$

Set $\mathcal{L}_{\mathcal{G}}^{0}=e \cdot \mathcal{H}_{\mathrm{dR}}^{1}\left(\mathcal{G} / \hat{\mathcal{H}}_{p}^{\text {unr }}\right)$ and $\omega_{\mathcal{G}}^{0}=e \cdot \underline{\omega}_{\mathcal{G}}$. Define $\Lambda_{\mathcal{G}}=H^{0}\left(\mathcal{H}_{p}^{\text {unr }}, \mathcal{L}_{\mathcal{G}}^{0}\right), \Lambda_{\mathcal{G}}^{*}=\Lambda_{\mathcal{G}} \otimes_{\mathcal{A}} \mathcal{A}^{*}$, $w_{\mathcal{G}}=H^{0}\left(\mathcal{H}_{p}^{\mathrm{unr}}, \underline{\omega}_{\mathcal{G}}^{0}\right), w_{\mathcal{G}}^{*}=w_{\mathcal{G}} \otimes_{\mathcal{A}} \mathcal{A}^{*}$. We have then an injective map of $\mathcal{A}^{*}$-algebras

$$
w_{\mathcal{G}}^{*} \hookrightarrow \Lambda_{\mathcal{G}}^{*}
$$

Theorem 1.1 The injection (5) of $\mathcal{A}^{*}$-algebras admits a canonical splitting $\Psi_{p}: \Lambda_{\mathcal{G}}^{*} \rightarrow w_{\mathcal{G}}^{*}$.

This result corresponds to Theorem 4.5 below. The main tool which is used to prove Theorem 1.1 is Drinfel'd interpretation of $\hat{\mathcal{H}}_{p}^{\text {unr }}$ as moduli space of special formal modules with quaternionic multiplication; following [43], we call these objects SFD-modules. We study the relative de Rham cohomology of the universal SFD-module $\mathcal{G} \rightarrow \hat{\mathcal{H}}_{p}^{\text {unr }}$ by means of techniques from [14, 23, 43]. The upshot of our analysis is an explicit description of the Gauss-Manin connection and the Kodaira-Spencer isomorphism for $\mathcal{G} \rightarrow \hat{\mathcal{H}}_{p}^{\text {unr }}$, once we apply to the relevant sheaves the projector $e$. This detailed study is contained in Sect. 4, which we believe is of independent interest and is the technical part of the paper. It should be noticed that related results on the splitting of the Hodge filtration have been obtained, even in greater generality, in $[22,40]$. Our result is in a way more explicit, but the price to pay is that it only works over the unramified upper half plane.

Using the splitting of the Hodge filtration in Theorem 1.1, we may then attach to $\Psi_{p}$ a $p$-adic Maass-Shimura operator

$$
\Theta_{p, k}: w_{\mathcal{G}, k}^{*} \longrightarrow w_{\mathcal{G}, k+2}^{*}
$$

where for each integer $t \geq 1$ we put $w_{\mathcal{G}, t}^{*}=\left(w_{\mathcal{G}}^{*}\right)^{\otimes t}$. Using Teitelbaum's description of $\mathcal{G}$, one can find a basis $\left\{d \tau, d \tau^{*}\right\}$ of $w_{\mathcal{G}}^{*}$ such that $\Psi_{p}\left(d \tau^{*}\right)=0$. For each integer $r \geq 1$, one may define the $r$-th iterate $\Theta_{p, k}^{r}: w_{\mathcal{G}, k}^{*} \rightarrow w_{\mathcal{G}, k+2 r}^{*}$ and compare it with the $r$-th iterate $\delta_{p, k}^{r}: \mathcal{A}^{*} \rightarrow \mathcal{A}^{*}$ of Franc $p$-adic Maass-Shimura operator defined in [15]. We prove that $\delta_{p, k}^{r}$ arises from the $p$-adic Maass-Shimura operator $\Theta_{p, k}^{r}$. More precisely, we have the following result (see (35) below). 
Corollary 1.2 There exists $t_{p} \in \mathbb{C}_{p}^{\times}$, such that for each $f \in \mathcal{A}^{*}$ and integers $k \geq 1$ and $r \geq 1$ we have

$$
\Theta_{p, k}^{r}\left(f(z) d \tau^{\otimes k}\right)=\frac{\delta_{p, k}^{r}(f)(z)}{t_{p}^{r}} d \tau^{\otimes(k+2 r)} .
$$

Remark 1.3 The $p$-adic number $t_{p}$ arises as a period comparing two pairings on the cohomology of SFD-modules, and can be understood as a $p$-adic analogue of the complex period $2 \pi i$. See (27) for details. It is independent of $f, k$ and $r$.

Fix an embedding $\overline{\mathbb{Q}} \hookrightarrow \overline{\mathbb{Q}}_{p}$; we say that a $p$-adic number $x \in \overline{\mathbb{Q}}_{p}$ is algebraic if it belongs to the image of this embedding, in which case we simply write $x \in \overline{\mathbb{Q}}$. Let $S_{k}^{\text {rig }}\left(\Gamma_{p}\right)$ be the $\mathbb{C}_{p}$-vector space of rigid analytic quaternionic modular forms of weight $k$ and level $\Gamma_{p}$; elements of $S_{k}^{\text {rig }}\left(\Gamma_{p}\right)$ are functions from $\mathcal{H}_{p}\left(\mathbb{C}_{p}\right)=\mathbb{C}_{p}-\mathbb{Q}_{p}$ to $\mathbb{C}_{p}$ which transform under the action of $\Gamma_{p}$ by the automorphic factor of weight $k$. We say that $f \in S_{k}^{\text {rig }}\left(\Gamma_{p}\right)$ is algebraic if it corresponds, via the Cerednik-Drinfel'd theorem, to an algebraic modular form of weight $k$ on $\mathcal{C}$ which is defined over $\overline{\mathbb{Q}}$ (see Sect. 2.5 for the notion of algebraic modular forms, and Sect. 5.2 for the comparison between rigid analytic and algebraic modular forms).

Corollary 1.4 Let $f \in S_{k}^{\mathrm{rig}}\left(\Gamma_{p}\right)$ be an algebraic modular form of weight $k$ and level $\Gamma_{p}$. Then for every CM point $z \in K \cap \mathcal{H}_{p}\left(\hat{\mathbb{Q}}_{p}^{\mathrm{unr}}\right)$, we have

$$
\frac{\delta_{p, k}^{r}(f)(z)}{t_{p}^{r}} \in \mathbb{Q} .
$$

As remarked above, Corollary 1.4 is the main result of Franc thesis [15], which he proves via an explicit approach following Shimura. Instead, we derive this result in Theorem 5.1 from a comparison between the values at CM points of our $p$-adic Maass-Shimura operator and the real analytic Maass-Shimura operator.

We explain now the connection with generalized Heegner cycles. These cycles were introduced in [4] with the aim of studying certain anticyclotomic $p$-adic $L$-functions. Generalized Heegner cycles have been also studied in the context of Shimura curves with good reduction at $p$ in [19], and in the context of Mumford curves in [30, 34]. Fix a false elliptic curve $A_{0}$ with $\mathrm{CM}$ by $\mathcal{O}_{K}$. For any isogeny $\varphi: A_{0} \rightarrow A$ of false elliptic curves, we construct a cycle $\Upsilon_{\varphi}$ in the Chow group $\mathrm{CH}^{m}\left(\mathcal{A} \times A_{0}\right)$ of the Chow motive $\mathcal{A} \times A_{0}$, where $m=n / 2$ with $n=k-2$. Brooks introduces in [19] a projector $\epsilon$ in the ring of correspondences of $X_{m}=\mathcal{A}^{m} \times A_{0}^{m}$, which defines the motive $\mathcal{D}=\left(X^{m}, \epsilon\right)$. The generalised Heegner cycle $\Delta_{\varphi}$ is the image of $\Upsilon_{\varphi}$ in $\mathrm{CH}^{m}(\mathcal{D})$ via this projector. We construct a $p$-adic Abel-Jacobi map

$$
\mathrm{AJ}_{p}: \mathrm{CH}^{m}(\mathcal{D}) \longrightarrow\left(S_{k}^{\mathrm{rig}}(\Gamma) \otimes \operatorname{Sym}^{m} e H_{\mathrm{dR}}^{1}\left(A_{0}\right)\right)^{\vee}
$$

where ${ }^{\vee}$ denotes linear dual (see Sect. 7.1 for details). We prove that the sheaf $\mathcal{L}_{\mathcal{G}}^{0}=$ $e \mathcal{H}_{\mathrm{dR}}^{1}\left(\mathcal{G} / \hat{\mathcal{H}}_{p}^{\mathrm{unr}}\right)$ is equipped with two canonical sections $\omega_{\text {can }}$ and $\eta_{\text {can }}$, such that $\omega_{\text {can }}$ is a generator of the invertible sheaf $\omega_{\mathcal{G}}^{0}$. Let $\omega_{f} \in w_{\mathcal{G}}^{0}$ be the $\Gamma$-invariant differential form associated with an algebraic modular form $f \in S_{k}^{\text {rig }}(\Gamma)$, and let $F_{f}$ denote its Coleman primitive satisfying $\nabla\left(F_{f}\right)=\omega_{f}$, where $\nabla$ is the Gauss-Manin connection. Denote $\langle$,$\rangle the$ Poincaré pairing on $\operatorname{Sym}^{n} e H_{\mathrm{dR}}^{1}\left(A_{z}\right)$, where $A_{z}$ is the fiber of $\mathcal{A}$ at $z$. Define the function

$$
H(z)=\left\langle F_{f}(z), \omega_{\text {can }}^{n}(z)\right\rangle .
$$


Theorem 1.5 Let $\varphi: A_{0} \rightarrow A$ be an isogeny of degree $d_{\varphi}$ prime to $N p$, and $z_{A}$ the point of $\mathcal{C}$ whose fiber is $A$. Then for each integer $j=n / 2, \ldots, n$ we have

$$
\frac{\delta_{p, k}^{n-j}(H)\left(z_{A}\right)}{t_{p}^{n-j}}=\frac{n !}{j ! \cdot d_{\varphi}^{j}} \cdot \mathrm{AJ}_{p}\left(\Delta_{\varphi}\right)\left(\omega_{f} \otimes \omega_{\mathrm{can}}^{j} \eta_{\mathrm{can}}^{n-j}\right)
$$

Theorem 1.5 relates the Maass-Shimura operator with generalised Heegner cycles, and corresponds to Corollary 7.2.

We finally make a remark on $p$-adic $L$-functions. It would be interesting to use our $p$-adic Maass-Shimura operator to construct $p$-adic $L$-functions interpolating special values of the complex $L$-function of $f$ twisted by Hecke characters as in $[1,4,19,28]$. We would like to come back to this problem in a future work.

\section{Algebraic de Rham cohomology of Shimura curves}

Throughout this section, let $k \geq 2$ be an even integer and $N \geq 1$ an integer. Fix an imaginary quadratic field $K / \mathbb{Q}$ of discriminant $D_{K}$ prime to $N$ and factor $N=N^{+} \cdot N^{-}$by requiring that all primes dividing $N^{+}$(respectively $N^{-}$) split in $K$ (respectively, are inert in $K$ ). Assume that $N^{-}$is a square-free of an odd number of primes, and let $p \nmid N$ be a prime number which is inert in $K$ (thus $N^{-} p$ is a square-free of an even number of primes). Fix also embeddings $\overline{\mathbb{Q}} \hookrightarrow \mathbb{C}$ and $\overline{\mathbb{Q}} \hookrightarrow \overline{\mathbb{Q}}_{\ell}$ for each prime number $\ell$.

\subsection{Quaternion algebras}

Let $\mathcal{B} / \mathbb{Q}$ be the indefinite quaternion algebra of discriminant $N^{-} p$. We need to fix a convenient basis for the $\mathbb{Q}$-algebra $\mathcal{B}$, called Hashimoto model. Denote $M=\mathbb{Q}\left(\sqrt{p_{0}}\right)$ the splitting field of the quadratic polynomial $X^{2}-p_{0}$, where $p_{0}$ if an auxiliary prime number fixed as in [37, Sect. 1.1] and [19, Sect. 2.1], such that:

(1) for all primes $\ell$ we have $\left(p_{0}, p N^{-}\right)_{\ell}=-1$ if and only if $\ell \mid p N^{-}$, where $(a, b)_{\ell}$ denotes the Hilbert symbol,

(2) all primes $\ell \mid N^{+}$are split in the real quadratic field $M=\mathbb{Q}\left(\sqrt{p_{0}}\right)$, where $\sqrt{p_{0}}$ is a square root of $p_{0}$ in $\overline{\mathbb{Q}}$.

The choice of $p_{0}$ fixes a $\mathbb{Q}$-basis of $\mathcal{B}$ as in $\left[18\right.$, Sect. 2] given by $\{\mathbf{1}, \mathbf{i}, \mathbf{j}, \mathbf{k}\}$ with $\mathbf{i}^{2}=-p N^{-}$, $\mathbf{j}^{2}=p_{0}, \mathbf{k}=\mathbf{i j}=-\mathbf{j i}$, and $\mathbf{1}$ the unit of $\mathcal{B}$; of course, if $x \in \mathbb{Q}$ we will often just write $x$ for $x \cdot \mathbf{1}$. Let $\mathcal{R}_{\max }$ be the maximal order of $\mathcal{B}$ which contains the $\mathbb{Z}$-span of this basis. Since $M$ splits $\mathcal{B}$, we have isomorphisms $\iota_{M}: \mathcal{B} \otimes_{\mathbb{Q}} M \simeq \mathrm{M}_{2}(M)$ and for place $v \nmid N^{-} p$ of $M$ we have $\iota_{M}\left(\mathcal{R}_{\max } \otimes_{\mathbb{Z}} \mathcal{O}_{M, v}\right)=\mathrm{M}_{2}\left(\mathcal{O}_{M, v}\right)$, where $\mathcal{O}_{M}$ is the ring of algebraic integers of $M$, and $\mathcal{O}_{M, v}$ is the $v$-adic completion of $\mathcal{O}_{M}$ [19, Lemma 2.1]. For each prime number $\ell \mid N^{+}$, choose a place $v$ of $M$ above $\ell$ with $M_{v}=\mathbb{Q}_{\ell}$. We thus obtain an isomorphism $\iota_{\ell}: \mathcal{B} \otimes_{\mathbb{Q}} \mathbb{Q}_{\ell} \simeq \mathrm{M}_{2}\left(\mathbb{Q}_{p}\right)$ such that $\iota_{\ell}\left(\mathcal{R}_{\max } \otimes_{\mathbb{Z}} \mathbb{Z}_{\ell}\right)=\mathrm{M}_{2}\left(\mathbb{Z}_{\ell}\right)$. Define $V_{1}\left(N^{+}\right)$to be the subgroup of $\mathcal{R}_{\max }$ consisting of elements $x$ such that $\iota_{\ell}(x) \equiv\left(\begin{array}{c}* * \\ 0\end{array}\right)\left(\bmod N^{+}\right)$. This subgroup is contained in the standard Eichler order $\mathcal{R} \subseteq \mathcal{R}_{\max }$ of level $N^{+}$. Let finally $\Gamma$ be the subgroup of elements $x \in \mathcal{R}^{\times}$having norm one and such that $i_{\ell}(x) \equiv\left(\begin{array}{ll}* * \\ 0 & 1\end{array}\right)\left(\bmod N^{+}\right)$ for all $\ell \mid N^{+}$. 


\subsection{Moduli problem}

A false elliptic curve A over a scheme $S$ is a an abelian scheme $A \rightarrow S$ of relative dimension 2 equipped with an embedding $\iota_{A}: \mathcal{R}_{\max } \hookrightarrow \operatorname{End}_{S}(A)$. An isogeny of false elliptic curves is an isogeny which commutes with the action of $\mathcal{R}_{\max }$. A full level $N^{+}$-structure on $A$ is an isomorphism of group schemes $\alpha_{A}: A\left[N^{+}\right] \simeq\left(\mathcal{R}_{\max } \otimes_{\mathbb{Z}}\left(\mathbb{Z} / N^{+} \mathbb{Z}\right)\right)_{S}$, where for any group $G$ we denote $G_{S}$ the constant group scheme $G$ over $S$. A level structure of $V_{1}\left(N^{+}\right)$-type is an equivalence class of full level $N^{+}$-structures under the (right) action of $V_{1}\left(N^{+}\right)$.

The moduli problem which associates to any $\mathbb{Z}[1 / N p]$-scheme $S$ the set of isomorphism classes of false elliptic curves equipped with a $V_{1}\left(N^{+}\right)$-level structure is representable by a smooth proper scheme $\mathcal{C}$ defined over $\operatorname{Spec}(\mathbb{Z}[1 / N p])$ [19, Theorem 2.2]. Let $\pi: \mathcal{A} \rightarrow \mathcal{C}$ be the universal false elliptic curve. For any $\mathbb{Z}[1 / N p]$-algebra $R$, let $\pi_{R}: \mathcal{A}_{R} \rightarrow \mathcal{C}_{R}$ be the base change of $\pi$ to $R$. We have $\Gamma \backslash \mathcal{H} \simeq \mathcal{C}_{\mathbb{C}}(\mathbb{C})$, where $\mathcal{H}$ is the complex upper half plane and $\Gamma$ acts on it by Moebius transformations.

\subsection{Algebraic de Rham cohomology}

We review some preliminaries on the algebraic de Rham cohomology of Shimura curves, including the Gauss-Manin connection and the Kodaira-Spencer map, referring for details to [27], and especially to [37, Sect. 2.1] for the case under consideration of Shimura curves.

We first recall some general notation. For any morphism of schemes $\phi: X \rightarrow S$, denote $\left(\Omega_{X / S}^{\bullet}, d_{X / S}^{\bullet}\right)$, or simply $\Omega_{X / S}^{\bullet}$ understanding the differentials $d_{X / S}^{\bullet}$, the complex of sheaves of relative differential forms for the morphism $\phi$. For a sheaf $\mathcal{F}$ of $\mathcal{O}_{X}$-modules over a scheme $X$, we denote $\mathcal{F}^{\vee}$ its $\mathcal{O}_{X}$-linear dual. If $\mathcal{F}$ is invertible, for an integer $k$ we let $\mathcal{F}^{\otimes k}$ denote the usual tensor product operation.

Fix a field $F$ of characteristic zero. The relative de Rham cohomology bundle for the morphism $\mathcal{A}_{F} \stackrel{\pi_{F}}{\rightarrow} \mathcal{C}_{F}$ is defined by

$$
\mathcal{H}_{\mathrm{dR}}^{q}\left(\mathcal{A}_{F} / \mathcal{C}_{F}\right)=\mathbb{R}^{q} \pi_{F *}\left(\Omega_{\mathcal{A}_{F} / \mathcal{C}_{F}}\right) .
$$

We first recall the construction of the Gauss-Manin connection. We have a canonical short exact sequence of locally free sheaves

$$
0 \longrightarrow \pi_{F}^{*}\left(\Omega_{\mathcal{C}_{F} / F}^{1}\right) \longrightarrow \Omega_{\mathcal{A}_{F} / \mathcal{C}_{F}}^{1} \longrightarrow \Omega_{\mathcal{A}_{F} / F}^{1} \longrightarrow 0
$$

(the exactness is because $\pi_{F}$ is smooth). This exact sequence induces maps

$$
\Omega_{\mathcal{A}_{F} / F}^{\bullet-i} \otimes_{\mathcal{O}_{\mathcal{A}_{F}}} \pi_{F}^{*}\left(\Omega_{\mathcal{C}_{F} / F}^{i}\right) \stackrel{\psi_{\mathcal{A}_{F} / F}^{i}}{\longrightarrow} \Omega_{\mathcal{A}_{F} / F}^{\bullet}
$$

for each integer $i$, defining a filtration $F^{i} \Omega_{\mathcal{A}_{F} / F}^{\bullet}=\operatorname{Im}\left(\psi_{\mathcal{A}_{F} / F}^{i}\right)$ on $\Omega_{\mathcal{A}_{F} / F}^{\bullet}$ with associated graded objects

$$
\operatorname{gr}^{i}\left(\Omega_{\mathcal{A}_{F} / F}^{\bullet}\right)=\Omega_{\mathcal{A}_{F} / F}^{\bullet-i} \otimes \mathcal{O}_{\mathcal{A}_{F}} \pi_{F}^{*}\left(\Omega_{\mathcal{C}_{F} / F}^{i}\right)
$$

Let $E_{i}^{p, q}$ denote the spectral sequence associated with this filtration. The $E_{1}^{p, q}$ terms are then given by

$$
E_{1}^{p, q} \simeq \Omega_{\mathcal{C}_{F} / F}^{p} \otimes_{\mathcal{O}_{\mathcal{C}_{F}}} \mathcal{H}_{\mathrm{dR}}^{q}\left(\mathcal{A}_{F} / \mathcal{C}_{F}\right)
$$


$[27,(7)]$. The Gauss-Manin connection

$$
\nabla: \mathcal{H}_{\mathrm{dR}}^{i}\left(\mathcal{A}_{F} / \mathcal{C}_{F}\right) \longrightarrow \Omega_{\mathcal{C}_{F} / F}^{1} \otimes_{\mathcal{O}_{\mathcal{C}_{F}}} \mathcal{H}_{\mathrm{dR}}^{i}\left(\mathcal{A}_{F} / \mathcal{C}_{F}\right)
$$

is then defined as the differential $d_{1}^{0, i}: E_{1}^{0, i} \rightarrow E_{1}^{1, i}$ in this spectral sequence.

We now recall various descriptions of the Kodaira-Spencer map. It is defined to be the boundary map

$$
\mathrm{KS}_{\mathcal{A}_{F} / \mathcal{C}_{F}}: \pi_{F *}\left(\Omega_{\mathcal{A}_{F} / \mathcal{C}_{F}}^{1}\right) \longrightarrow \mathbb{R}^{1} \pi_{F *}\left(\pi_{F}^{*}\left(\Omega_{\mathcal{C}_{F} / F}^{1}\right)\right)
$$

in the long exact sequence of derived functors obtained from (6). It can also be reconstructed from the Gauss-Manin connection as follows. Let $\pi_{F}^{\vee}: \mathcal{A}_{F}^{\vee} \rightarrow \mathcal{C}_{F}$ denote the dual abelian variety. By a result of Buzzard ([9, Sect. 1], see also [19, page 4183]), it is known that the abelian surface $\mathcal{A}_{F}$ is equipped with a canonical principal polarization $\iota_{\mathcal{A}_{F}}: \mathcal{A}_{F} \simeq \mathcal{A}_{F}^{\vee}$ over $\mathcal{C}_{F}$, which we use to identify $\mathcal{A}_{F}^{\vee}$ and $\mathcal{A}_{F}$ in the following without explicit mentioning it; we recall that this polarization is characterized by the fact that the associated Rosati involution in $\operatorname{End}_{\mathcal{C}_{F}}\left(\mathcal{A}_{F}\right)$ restricted to the image of $\mathcal{R}_{\text {max }}$ via the map $\mathcal{R}_{\max } \hookrightarrow \operatorname{End}_{\mathcal{C}_{F}}\left(\mathcal{A}_{F}\right)$ coincides with the involution $x \mapsto x^{\dagger}$ of $\mathcal{R}_{\text {max }}$, defined by $x^{\dagger}=\mathbf{i}^{-1} \bar{b} \mathbf{i}$ (as usual, if $x=a+b \mathbf{i}+c \mathbf{j}+d \mathbf{k}$, then $\bar{x}=a-b \mathbf{i}-c \mathbf{j}-d \mathbf{k})$. Using the principal polarization and the isomorphism between $\mathbb{R}^{1} \pi_{F *}\left(\Omega_{\mathcal{A}_{F} / F}^{1}\right)$ and the tangent bundle of $\mathcal{A}_{F}^{\vee}$, the Hodge exact sequence can be written as

$$
0 \longrightarrow \pi_{F *}\left(\Omega_{\mathcal{A}_{F} / \mathcal{C}_{F}}^{1}\right) \longrightarrow \mathcal{H}_{\mathrm{dR}}^{1}\left(\mathcal{A}_{F} / \mathcal{C}_{F}\right) \longrightarrow\left(\pi_{F *}\left(\Omega_{\mathcal{A}_{F} / \mathcal{C}_{F}}^{1}\right)\right)^{\vee} \longrightarrow 0
$$

(cf. [37, (2.2)], [19, Sect. 2.6]). The Kodaira-Spencer map can be defined using the GaussManin connection as the composition

$$
\begin{aligned}
& \mathrm{KS}_{\mathcal{A}_{F} / \mathcal{C}_{F}}: \pi_{F *}\left(\Omega_{\mathcal{A}_{F} / \mathcal{C}_{F}}^{1}\right) \stackrel{(7)}{\longrightarrow} \mathcal{H}_{\mathrm{dR}}^{1}\left(\mathcal{A}_{F} / \mathcal{C}_{F}\right) \stackrel{\nabla}{\longrightarrow} \mathcal{H}_{\mathrm{dR}}^{i}\left(\mathcal{A}_{F} / \mathcal{C}_{F}\right) \otimes \mathcal{O}_{\mathcal{C}_{F}} \Omega_{\mathcal{C}_{F} / F}^{1} \stackrel{(7)}{\longrightarrow} \\
& \stackrel{(7)}{\longrightarrow}\left(\pi_{F *}\left(\Omega_{\mathcal{A}_{F} / \mathcal{C}_{F}}\right)\right)^{\vee} \otimes \mathcal{O}_{\mathcal{C}_{F}} \Omega_{\mathcal{C}_{F} / F}^{1}
\end{aligned}
$$

in which the first and the last map come from the Hodge exact sequence (7). Therefore the Kodaira-Spencer map can also be seen as a map of $\mathcal{O}_{\mathcal{C}_{F}}$-modules, denoted again with the same symbol,

$$
\mathrm{KS}_{\mathcal{A}_{F} / \mathcal{C}_{F}}: \pi_{F *}\left(\Omega_{\mathcal{A}_{F} / \mathcal{C}_{F}}^{1}\right)^{\otimes 2} \longrightarrow \Omega_{\mathcal{C}_{F} / F}^{1}
$$

\subsection{Idempotents and line bundles}

Let

$$
e=\frac{1}{2}\left(\mathbf{1} \otimes 1+\frac{1}{p_{0}} \mathbf{j} \otimes \sqrt{p_{0}}\right) \in \mathcal{R}_{M}=\mathcal{R}_{\max } \otimes_{\mathbb{Z}} \mathcal{O}_{M}\left[1 /\left(2 p_{0}\right)\right]
$$

be the idempotent in [37, (1.10)], [19, Sect. 2.1], where $\mathcal{O}_{M}$ is the ring of integers of $M$.

Suppose we have an embedding $M \hookrightarrow F$, allowing us to identify $M$ with a subfield of $F$; in the cases we are interested in, either $F \subseteq \overline{\mathbb{Q}}$ (and then we require that $F$ contains $M$ ), or $F=\mathbb{C}$ (and then we view $M \hookrightarrow \mathbb{C}$ via the fixed embedding $\overline{\mathbb{Q}} \hookrightarrow \mathbb{C}$ ) or $F \subseteq \overline{\mathbb{Q}}_{p}$ (and then we require that $F$ contains the image of $M$ via the fixed embedding $\overline{\mathbb{Q}} \hookrightarrow \overline{\mathbb{Q}}_{p}$ ). 
Since $M$ is contained in $F$, we have an action of $\mathcal{R}_{M}$ on the sheaves $\pi_{F *}\left(\Omega_{\mathcal{A}_{F} / \mathcal{C}_{F}}^{1}\right)$ and $\mathcal{H}_{\mathrm{dR}}^{1}\left(\mathcal{A}_{F} / \mathcal{C}_{F}\right)$, and we may therefore define the invertible sheaf of $\mathcal{O}_{\mathcal{C}_{F}}$-modules

$$
\underline{\omega}_{F}=e \cdot \pi_{F *}\left(\Omega_{\mathcal{A}_{F} / \mathcal{C}_{F}}^{1}\right)
$$

and the sheaf of $\mathcal{O}_{\mathcal{C}_{F}}$-modules

$$
\mathcal{L}_{F}=e \cdot \mathcal{H}_{\mathrm{dR}}^{1}\left(\mathcal{A}_{F} / \mathcal{C}_{F}\right) .
$$

Using that $e$ is fixed by the Rosati involution, the Hodge exact sequence (7) becomes

$$
0 \longrightarrow \underline{\omega}_{F} \longrightarrow \mathcal{L}_{F} \longrightarrow \underline{\omega}_{F}^{-1} \longrightarrow 0
$$

(see [19, Sect. 2.6]). For any integer $n \geq 1$, define

$$
\mathcal{L}_{F, n}=\operatorname{Sym}^{n}\left(\mathcal{L}_{F}\right) .
$$

The Gauss-Manin connection is compatible with the quaternionic action [37, Proposition 2.2]. Therefore, restricting to $\mathcal{L}_{F, 1}$ and using the Leibniz rule (see for example [19, Sect. 3.2]), the Gauss-Manin connection defines a connection

$$
\nabla_{n}: \mathcal{L}_{F, n} \longrightarrow \mathcal{L}_{F, n} \otimes \Omega_{\mathcal{C}_{F} / F}^{1} .
$$

By [37, Theorem 2.5], restricting the Kodaira-Spencer map to $\underline{\omega}_{F}^{\otimes 2}$ gives an isomorphism

$$
\mathrm{KS}_{F}: \underline{\omega}_{F}^{\otimes 2} \stackrel{\sim}{\longrightarrow} \Omega_{\mathcal{C}_{F} / F}^{1} .
$$

We may then define a map $\tilde{\nabla}_{n}: \mathcal{L}_{F, n} \rightarrow \mathcal{L}_{F, n+2}$ by the composition

$$
\tilde{\nabla}_{n}: \mathcal{L}_{F, n} \stackrel{\nabla_{n}}{\longrightarrow} \mathcal{L}_{F, n} \otimes_{\mathcal{O}_{\mathcal{C}_{L}}} \Omega_{\mathcal{C}_{F} / F}^{1} \stackrel{\mathrm{id} \otimes \mathrm{KS}_{F}^{-1}}{\longrightarrow} \mathcal{L}_{F, n} \otimes_{\mathcal{O}_{\mathcal{C}_{L}}} \underline{\omega}_{F}^{\otimes 2} \longrightarrow \mathcal{L}_{F, n} \otimes_{\mathcal{O}_{\mathcal{C}_{L}}} \mathcal{L}_{F, 2} \longrightarrow \mathcal{L}_{F, n+2}
$$

where the last map is the product map in the symmetric algebras.

\subsection{Algebraic modular forms}

As in the previous section, let $F$ e a field of characteristic 0 . For any $F$-algebra $R$, we define the $R$-algebra

$$
S_{k}^{\mathrm{alg}}(\Gamma, R)=H^{0}\left(\mathcal{C}_{R}, \underline{\omega}_{R}^{\otimes k}\right)
$$

of algebraic modular forms of weight $k$ and level $V_{1}\left(N^{+}\right)$over $R$. One can show [19, Sect. $3.1]$ that the $R$-algebra $S_{k}^{\text {alg }}(\Gamma, R)$ can be alternatively described in modular terms. Let $R^{\prime}$ be an $R$ algebra. A test triple over $R^{\prime}$ is a triplet $\left(A^{\prime}, t^{\prime}, \omega^{\prime}\right)$ consisting of a false elliptic curve $A^{\prime} / R^{\prime}$, a $V_{1}\left(N^{+}\right)$-level structure $t^{\prime}$ and a global section $\omega^{\prime}$ of $\underline{\omega}_{A^{\prime} / R^{\prime}}$. An isomorphism of test triples $\left(A^{\prime}, t^{\prime}, \omega^{\prime}\right)$ and $\left(A^{\prime \prime}, t^{\prime \prime}, \omega^{\prime \prime}\right)$ is an isomorphism of false elliptic curves $\phi: A^{\prime} \rightarrow A^{\prime \prime}$ such that $\phi\left(t^{\prime}\right)=t^{\prime \prime}$ and $\phi^{*}\left(\omega^{\prime \prime}\right)=\omega^{\prime}$. A test pair over $R^{\prime}$ is a pair $\left(A^{\prime}, t^{\prime}\right)$ obtained from a test triplet by forgetting the datum of the global section. Then one can identify global sections of $\underline{\omega}_{R}^{\otimes k}$ with:

(1) A rule $F$ which assigns, to each $R$-algebra $R^{\prime}$ and each isomorphism class of test triplets $\left(A^{\prime}, t^{\prime}, \omega^{\prime}\right)$ over $R^{\prime}$, an element $F\left(A^{\prime}, t^{\prime}, \omega^{\prime}\right) \in R^{\prime}$, subject to the base change axiom (for all maps of $R$-algebras $\phi: R^{\prime} \rightarrow R^{\prime \prime}$, we have $F\left(A^{\prime}, t^{\prime}, \phi^{*}\left(\omega^{\prime \prime}\right)\right)=F\left(A^{\prime \prime}, \phi\left(t^{\prime}\right), \omega^{\prime \prime}\right)$, where $A^{\prime}$ is the base change of $A^{\prime \prime}$ via $\phi$ ) and the weight $k$ condition (for all $\lambda \in\left(R^{\prime}\right)^{\times}$, we have $\left.F\left(A^{\prime}, t^{\prime}, \lambda \omega^{\prime}\right)=\lambda^{-k} F\left(A^{\prime}, t^{\prime}, \omega^{\prime}\right)\right)$ [19, Definition 3.2]. 
(2) A rule $F$ which assigns to each $R$-algebra $R^{\prime}$ and each isomorphism class of test pairs $\left(A^{\prime}, t^{\prime}\right)$ over $R^{\prime}$, a translation invariant section $F\left(A^{\prime}, t^{\prime}\right) \in \underline{\omega}_{A^{\prime} / R^{\prime}}^{\otimes k}$ subject to the base change axiom (for all maps of $R$-algebras $\phi: R^{\prime} \rightarrow R^{\prime \prime}$, we have the relation $F\left(A^{\prime}, t^{\prime}\right)=$ $\phi^{*}\left(F\left(A^{\prime \prime}, \phi\left(t^{\prime}\right)\right)\right.$, where $A^{\prime}$ is the base change of $A^{\prime \prime}$ via $\left.\phi\right)$ [19, Definition 3.3].

Let us make the relations between these definitions more explicit [19, page 4193]. Given a global section $f \in H^{0}\left(\mathcal{C}_{R}, \underline{\omega}_{R}^{\otimes k}\right)$, we get a function as in (2) above associating to each test pair $\left(A^{\prime}, t^{\prime}\right)$ over $R^{\prime}$ the point $x_{\left(A^{\prime}, t^{\prime}\right)} \in \mathcal{C}_{R}\left(R^{\prime}\right)$, and taking the value of $f$ at $x_{\left(A^{\prime}, t^{\prime}\right)}$; if $F$ is as in (2), we get a function $G$ on test triples $\left(A^{\prime}, t^{\prime}, \omega^{\prime}\right)$ over $R^{\prime}$ as in (1) by the formula $F\left(A^{\prime}, t^{\prime}\right)=G\left(A^{\prime}, t^{\prime}, \omega^{\prime}\right) \omega^{\otimes k}$ where $\omega \in \underline{\omega}_{A^{\prime} / R^{\prime}}$ is the choice of any translation invariant global section.

\section{Special values of $L$-series}

In this section we review the work of Brooks [19] expressing special values of certain $L$ functions of modular forms in terms of CM-values of the Maass-Shimura operator applied to the modular form in question.

\subsection{The real analytic Maass-Shimura operator}

We denote $\left(X, \mathcal{O}_{X}\right) \rightsquigarrow\left(X^{\text {an }}, \mathcal{O}_{X}^{\text {an }}\right)$ the analytification functor which takes a scheme of finite type over $\mathbb{C}$ to its associated complex analytic space. For each sheaf $\mathcal{F}$ of $\mathcal{O}_{X}$-modules on $X$, we also denote $\mathcal{F}^{\text {an }}$ the analytification of $\mathcal{F}$, and for each morphism $\varphi: \mathcal{F} \rightarrow \mathcal{G}$ of $\mathcal{O}_{X}$-modules, we let $\varphi^{\text {an }}: \mathcal{F}^{\text {an }} \rightarrow \mathcal{G}^{\text {an }}$ the corresponding morphism of analytic sheaves. If $\left(X, \mathcal{O}_{X}\right)$ is an analytic space, we denote $\mathcal{O}_{X}^{\text {ran }}$ the ring of real analytic functions on $X$; this is a sheaf of $\mathcal{O}_{X}$-modules, and for any sheaf $\mathcal{F}$ of $\mathcal{O}_{X}$-modules, we let $\mathcal{F}^{\text {ran }}=\mathcal{F} \otimes \mathcal{O}_{X} \mathcal{O}_{X}^{\text {ran }}$; when $\mathcal{F}=\mathcal{F}^{\text {an }}$, we simplify the notation by writing $\mathcal{F}^{\text {ran }}$ instead of $\left(\mathcal{F}^{\text {an }}\right)^{\text {ran }}$.

Since $\mathcal{C}_{\mathbb{C}}$ is proper and smooth over $\mathbb{C}$, the analytification functor $\mathcal{F} \rightsquigarrow \mathcal{F}^{\text {an }}$ induces an equivalence of categories between the category of coherent sheaves $\mathcal{C}_{\mathbb{C}}$ and the category of analytic coherent sheaves of $\mathcal{O}_{\mathcal{C}_{\mathbb{C}}}^{\text {an }}$-modules. Also, the analytic sheaf obtained from the sheaf of algebraic de Rham cohomology $\mathcal{H}_{\mathrm{dR}}^{i}\left(\mathcal{A}_{\mathbb{C}} / \mathcal{C}_{\mathbb{C}}\right)$ coincides with the derived functor $\mathbb{R}^{1} \pi_{\mathbb{C} *}^{\text {an }}\left(\Omega_{\mathcal{A}_{\mathbb{C}}^{\text {an }} / \mathcal{C}_{\mathbb{C}}^{\text {an }}}^{1}\right)$ in the category of analytic sheaves over $\mathcal{C}_{\mathbb{C}}^{\text {an }}[41$, Theorem 1$]$.

Hodge theory gives a splitting

$$
\mathcal{H}_{\mathrm{dR}}^{1}\left(\mathcal{A}_{\mathbb{C}} / \mathcal{C}_{\mathbb{C}}\right)^{\mathrm{ran}} \longrightarrow\left(\pi_{\mathbb{C} *}\left(\Omega_{\mathcal{A}_{\mathbb{C}} / \mathcal{C}_{\mathbb{C}}}\right)\right)^{\mathrm{ran}}
$$

of the corresponding Hodge exact sequence of real analytic sheaves obtained from (7). Since this splitting is the identity on the image of $\left(\pi_{\mathbb{C} *}\left(\Omega_{\mathcal{A}_{\mathbb{C}} / \mathcal{C}_{\mathbb{C}}}\right)\right)^{\text {ran }}$ in $\mathcal{H}_{\mathrm{dR}}^{1}\left(\mathcal{A}_{\mathbb{C}} / \mathcal{C}_{\mathbb{C}}\right)^{\text {ran }}$, it gives rise to a map $\Psi_{\infty}: \mathcal{L}_{\mathbb{C}, 1}^{\text {ran }} \rightarrow \underline{\omega}_{\mathbb{C}}^{\text {ran }}$ (cf. [37, Proposition 2.8]). We may then consider the induced maps $\Psi_{\infty, n}: \mathcal{L}_{\mathbb{C}, n}^{\text {ran }} \rightarrow\left(\underline{\omega}_{\mathbb{C}}^{\otimes n}\right)^{\text {ran }}$ for any integer $n \geq 1$. Further, the map $\tilde{\nabla}_{n}$ gives rise to a map $\tilde{\nabla}_{n}^{\text {ran }}: \mathcal{L}_{\mathbb{C}, n}^{\text {ran }} \rightarrow \mathcal{L}_{\mathbb{C}, n+2}^{\text {ran }}$ of real analytic sheaves. The composition

$$
\Theta_{\infty, n}:\left(\underline{\omega}_{\mathbb{C}}^{\otimes n}\right)^{\mathrm{ran}} \longrightarrow \mathcal{L}_{\mathbb{C}, n}^{\mathrm{ran}} \stackrel{\tilde{\nabla}_{n}^{\mathrm{ran}}}{\longrightarrow} \mathcal{L}_{\mathbb{C}, n+2}^{\mathrm{ran}} \stackrel{\Psi_{\infty, n}}{\longrightarrow}\left(\underline{\omega}_{\mathbb{C}}^{\otimes n+2}\right)^{\mathrm{ran}}
$$

is the real-analytic Shimura-Maas operator.

The effect of $\Theta_{\infty, n}$ on modular forms is described in [19, Proposition 3.4] and [37, Proposition 2.9]. Denote $\Gamma=\Gamma_{1}\left(N^{+}\right)$the subgroup of $\mathcal{B}^{\times} \cap V_{1}\left(N^{+}\right)$consisting of elements 
of norm equal to 1 . Fix an isomorphism $\mathcal{B} \otimes_{\mathbb{Q}} \mathbb{R} \simeq \mathrm{M}_{2}(\mathbb{R})$ and denote $\Gamma_{\infty}$ the image of $\Gamma$ in $\mathrm{GL}_{2}(\mathbb{R})$. Let $S_{k}\left(\Gamma_{\infty}\right)$ denote the $\mathbb{C}$-vector space of holomorphic modular forms of weight $k$ and level $\Gamma_{\infty}$ consisting of those holomorphic functions on $\mathcal{H}_{\infty}$, the complex upper half plane, such that $f(\gamma(z))=j(\gamma, z)^{k} f(z)$ for all $\gamma \in \Gamma_{\infty}$; here $\Gamma_{\infty}$ acts on $\mathcal{H}_{\infty}$ by fractional linear transformations via the map $\mathcal{B} \hookrightarrow \mathcal{B} \otimes \mathbb{Q} \mathbb{R} \simeq \mathrm{M}_{2}(\mathbb{R})$. We have (cf. [19, Sect. 2.7])

$$
S_{k}\left(\Gamma_{\infty}\right) \simeq H^{0}\left(\mathcal{C}_{\mathbb{C}}^{\mathrm{an}},\left(\underline{\omega}_{\mathbb{C}}^{\otimes k}\right)^{\mathrm{an}}\right)
$$

Define the space $S_{k}^{\text {ran }}\left(\Gamma_{\infty}\right)$ of real analytic modular forms of level $\Gamma_{\infty}$ and weight $k$ to be the $\mathbb{C}$-vector space of real analytic functions $f: \mathcal{H}_{\infty} \rightarrow \mathbb{C}$ such that $f(\gamma(z))=j(\gamma, z)^{k} f(z)$ for all $\gamma \in \Gamma_{\infty}$. One then has

$$
S_{k}^{\mathrm{ran}}\left(\Gamma_{\infty}\right) \simeq H^{0}\left(\mathcal{C}_{\mathbb{C}}^{\mathrm{an}},\left(\underline{\omega}_{\mathbb{C}}^{\otimes k}\right)^{\mathrm{ran}}\right)
$$

The operator $\Theta_{\infty, k}$ gives then rise to a map $\delta_{\infty, k}: S_{k}\left(\Gamma_{\infty}\right) \rightarrow S_{k+2}^{\mathrm{ran}}\left(\Gamma_{\infty}\right)$ and we have

$$
\delta_{\infty, k}(f)(z)=\frac{1}{2 \pi i}\left(\frac{d}{d z}+\frac{k}{z+\bar{z}}\right) f(z) .
$$

\subsection{CM points and triples}

Fix an embedding $\overline{\mathbb{Q}} \hookrightarrow \mathbb{C}$. For any embedding $\varphi: K \hookrightarrow \mathcal{B}$ there exists a unique $\tau \in \mathcal{H}$ such that $\iota_{\infty}\left(\varphi\left(K^{\times}\right)\right)(\tau)=\tau$. The additive map $K \hookrightarrow \mathbb{C}$ defined by $\alpha \mapsto j\left(\iota_{\infty}(\varphi(\alpha)), \tau\right)$ gives an embedding $K \hookrightarrow \mathbb{C}$; we say that $\varphi$ is normalized if $\alpha \mapsto j\left(\iota_{\infty}(\varphi(\alpha)), \tau\right)$ is the identity (with respect to our fixed embedding $\overline{\mathbb{Q}} \hookrightarrow \mathbb{C}$ ).

We say that $\tau \in \mathcal{H}$ is a $C M$ point if there exists an embedding $\varphi: K \hookrightarrow \mathcal{B}$ which has $\tau$ as fixed point as above, and that a $\mathrm{CM}$ point $\tau$ is normalized if $\varphi$ is normalized. Finally, we say that a CM point $\tau \in \mathcal{H}$ is a Heegner point if $\varphi\left(\mathcal{O}_{K}\right) \subseteq \mathcal{R}$ [19, Sect. 2.4 and page 4188].

Fix a CM point $\tau$ corresponding to an embedding $\varphi: K \hookrightarrow \mathcal{B}$. Let $\mathfrak{a}$ be an integral ideal of $\mathcal{O}_{K}$, and define the $\mathcal{R}_{\max }$-ideal $\mathfrak{a}_{\mathcal{B}}=\mathcal{R}_{\max } \cdot \varphi(\mathfrak{a})$. This ideal is principal, generated by an element $\alpha=\alpha_{\mathfrak{a}} \in \mathcal{B}$. Right multiplication by $\alpha$ gives an isogeny $A_{\tau} \rightarrow A_{\alpha^{-1} \tau}$, whose kernel is $A_{\tau}[\mathfrak{a}]$. Let $\Gamma_{\max }$ be the subgroup of $\mathcal{R}_{\max }^{\times}$consisting of elements of norm equal to 1 . The image of $\alpha \tau$ by the canonical projection map $\rho_{\max }: \mathcal{H} \rightarrow \Gamma_{\max } \backslash \mathcal{H}$ does not depend on the choice of the representative $\alpha$, and therefore one may write $A_{\mathfrak{a} \star \tau}$ for the corresponding abelian surface. Shimura's reciprocity law states that $\rho_{\max }(\tau)$ is defined over the Hilbert class field $H$ of $K$, and that $\rho_{\max }(\tau)^{\left(\mathfrak{a}^{-1}, H / K\right)}=\rho_{\max }(\mathfrak{a} \star \tau)$, where $\left(\mathfrak{a}^{-1}, H / K\right)$ denotes the Artin symbol.

Fix a primitive $N^{+}$-root of unity $\zeta$. Fix a normalized Heegner point $\tau$, and fix a point $P_{\tau} \in A_{\tau}\left[N^{+}\right]$of exact order $N^{+}$such that $e \cdot P=P$. Let $\left(A_{\tau}, P_{\tau}\right)$ denote the point on $\mathcal{C}(F)$ corresponding to the level structure $\mu_{N^{+}} \times \mu_{N^{+}} \simeq \mathbb{Z} / N^{+} \mathbb{Z} \times \mathbb{Z} / N^{+} \mathbb{Z} \rightarrow A_{\tau}\left[N^{+}\right]$which takes $(1,0) \in \mathbb{Z} / N^{+} \mathbb{Z} \times \mathbb{Z} / N^{+} \mathbb{Z}$ to $P_{\tau}$. A $C M$ triple is an isomorphism class of triples $\left(A_{\tau}, P_{\tau}, \omega_{\tau}\right)$ with $\left(A_{\tau}, P_{\tau}\right)$ as above and a non-vanishing section $\omega_{\tau}$ in $e \cdot \Omega_{A_{\tau} / F}$.

There is an action of $\mathrm{Cl}\left(\mathcal{O}_{K}\right)$ on the set of $\mathrm{CM}$ triples, given by

$$
\mathfrak{a} \star\left(A_{\tau}, P_{\tau}, \pi^{*}(\omega)\right)=\left(A_{\tau} / A_{\tau}[\mathfrak{a}], \pi\left(P_{\tau}\right), \omega\right)
$$

where $\pi: A_{\tau} \rightarrow A_{\tau} / A_{\tau}[\mathfrak{a}]$ is the canonical projection. 


\subsection{Special value formulas}

Fix a CM triple $(A, P, \omega)=\left(A_{\tau}, P_{\tau}, \omega_{\tau}\right)$ with $\omega$ defined over $H$, the Hilbert class field of $K$; recall that $A$ is also defined over $H$.

The complex structure $J_{\tau}$ on $\mathrm{M}_{2}(\mathbb{R})$ defines a differential form $\omega_{\mathbb{C}}=J_{\tau}^{*}\left(2 \pi i d z_{1}\right)$, and let $\Omega_{\infty} \in \mathbb{C}$ be defined by $\omega=\Omega_{\infty} \cdot \omega_{\mathbb{C}}$; clearly, different choices of $\omega$ correspond to changing $\Omega_{\infty}$ by a multiple in $H$.

We now let $f$ be a modular form of weight $k$, level $\Gamma_{1}\left(N^{+}\right) \cap \Gamma_{0}\left(N^{-}\right)$, and character $\varepsilon_{f}$, and let $f^{\mathrm{JL}}$ be the modular form on the Shimura curve $\mathcal{C}_{\mathbb{C}}$ associated with $f$ by the Jacquet-Langlands correspondence. We can normalise the choice of $f^{\mathrm{JL}}$ so that the ratio $\langle f, f\rangle /\left\langle f^{\mathrm{JL}}, f^{\mathrm{JL}}\right\rangle$ belongs to $K[19$, Sect. 2.7 and page 4232].

Let $\Sigma^{(2)}$ be the set of Hecke characters $\chi$ of $K$ of infinite type $\left(\ell_{1}, \ell_{2}\right)$ with $\ell_{1} \geq k$ and $\ell_{2} \leq 0$. We say that $\chi \in \Sigma^{(2)}$ is central critical if $\ell_{1}+\ell_{2}=k$, so that the infinite type of $\chi$ is $(k+j,-j)$ for some integer $j \geq 0$. Denote $\Sigma_{\mathrm{cc}}^{(2)}$ the subset of $\Sigma^{(2)}$ consisting of central critical characters.

For each positive integer $j$, let $\delta_{\infty, k}^{j}: S_{k}^{\mathrm{ran}}\left(\Gamma_{\infty}\right) \rightarrow D_{k+2 j}^{\mathrm{ran}}\left(\Gamma_{\infty}\right)$ denote the $j$-th iterate of the Shimura-Mass operator defined by

$$
\delta_{\infty, k}^{j}=\delta_{\infty, k+2(j-1)} \circ \cdots \circ \delta_{\infty, k+2} \circ \delta_{\infty, k} .
$$

For any Hecke character, one may consider the $L$-function $L\left(f, \chi^{-1}, s\right)$, and for $\chi \in \Sigma_{\mathrm{cc}}^{(2)}$ central critical define the algebraic part $L_{\mathrm{alg}}\left(f, \chi^{-1}\right)$ of its special value at $s=0$ as in [19, Proposition 8.7]. By [19, Proposition 8.7], if $\chi \in \Sigma_{\mathrm{cc}}^{(2)}$ then $L_{\mathrm{alg}}\left(f, \chi^{-1}\right) \in \overline{\mathbb{Q}}$, and we have

$$
L_{\mathrm{alg}}\left(f, \chi^{-1}\right)=\left(\sum_{\mathfrak{a} \in \mathrm{Cl}\left(\mathcal{O}_{K}\right)} \chi_{j}^{-1}(\mathfrak{a}) \cdot \delta_{\infty, k}^{j}\left(f^{\mathrm{JL}}\right)(\mathfrak{a} \star(A, t, \omega))\right)^{2}
$$

where $\chi_{j}=\chi \cdot \mathrm{nr}^{-j}$ and $\mathrm{nr}$ is the norm map on ideals of $\mathcal{O}_{K}$. In this formula we view the real analytic modular $\delta_{\infty, k}\left(f^{\mathrm{JL}}\right)$ as a function on test triplets, as in [19, Proposition 8.5] via (12) (see also the discussion in [4, page 1094] in the $\mathrm{GL}_{2}$ case).

For each ideal class $\mathfrak{a}$ in $\mathrm{Cl}\left(\mathcal{O}_{K}\right)$, let $\alpha_{\mathfrak{a}}$ be the corresponding element in $\mathcal{B}$, as in Sect. 3.2. Then using the dictionary between real analytic forms as functions on $\mathcal{H}$ or functions on test triples, and recalling that $A=A_{\tau}$ for a normalized Heegner point $\tau$, we have

$$
L_{\text {alg }}\left(f, \chi^{-1}\right)=\left(\Omega_{\infty}^{k+2 j} \cdot \sum_{\mathfrak{a} \in \operatorname{Cl}\left(\mathcal{O}_{K}\right)} \chi_{j}^{-1}(\mathfrak{a}) \cdot \delta_{\infty, k}^{j}\left(f^{\mathrm{JL}}\right)\left(\alpha_{\mathfrak{a}} \cdot \tau\right)\right)^{2} .
$$

In this formula we view $\delta_{\infty, k}^{j}\left(f^{\mathrm{JL}}\right)$ as a function on $\mathcal{H}$.

\section{The Maass-Shimura operator on the $p$-adic upper half plane}

In this section we define a $p$-adic Maass-Shimura operator in the context of Drinfel'd upper half plane. These results will be used in the next section to define a $p$-adic Maass-Shimura operator on Shimura curves, whose values at CM points will be compared with their complex analogue. As in the complex case, we will see that this operator plays a special role in defining $p$-adic $L$-functions. 
Let $\hat{\mathcal{H}}_{p}$ denote Drinfel'd $p$-adic upper half plane; this is a $\mathbb{Z}_{p}$-formal scheme, and we denote $\mathcal{H}_{p}$ its generic fiber, which is a $\mathbb{Q}_{p}$-rigid space [3, Chapitre I].

\subsection{Drinfel'd Theorem}

Denote $D$ the unique division quaternion algebra over $\mathbb{Q}_{p}$, and let $\mathcal{O}_{D}$ be its maximal order. The unramified quadratic extension $\mathbb{Q}_{p^{2}}$ of $\mathbb{Q}_{p}$ can be embedded in $D$, and in the following we will see it as a maximal commutative subfield of $D$ without explicitly mentioning it. Let $\sigma$ denote the absolute Frobenius automorphism of $\operatorname{Gal}\left(\mathbb{Q}_{p}^{\text {unr }} / \mathbb{Q}_{p}\right)$. If fix an element $\Pi \in \mathcal{O}_{D}$ such that $\Pi^{2}=p$ and $\Pi x=\sigma(x) \Pi$ for $x \in \mathbb{Q}_{p^{2}}$, then $D=\mathbb{Q}_{p^{2}}[\Pi]$. We will denote $x \mapsto \bar{x}$ the restriction of $\sigma$ to $\operatorname{Gal}\left(\mathbb{Q}_{p^{2}} / \mathbb{Q}_{p}\right)$.

For any $\mathbb{Z}_{p}$-algebra $B$, a formal $\mathcal{O}_{D}$-module over $B$ is a commutative 2-dimensional formal group $G$ over $B$ equipped with an embedding $\iota_{G}: \mathcal{O}_{D} \hookrightarrow \operatorname{End}(G)$. A formal $\mathcal{O}_{D^{-}}$ module is said to be special if for each geometric point $P$ of $\operatorname{Spec}(B / p B)$, the representation of $\mathcal{O}_{D} / \Pi \mathcal{O}_{D}$ on the tangent space $\operatorname{Lie}\left(G_{P}\right)$ of $G_{P}=G \times k_{P}$ is the sum of two distinct characters of $\mathcal{O}_{D} / \Pi \mathcal{O}_{D}$, where $k_{P}$ is the residue field of $P$; see [43, Definition 1] for more details on this definition. By an SFD-module over $B$, we mean a special formal $\mathcal{O}_{D}$-module over $B$. If $G$ is a SFD-module over a $\mathbb{Z}_{p}$-algebra $B$ in which $p$ is nilpotent, we denote $\mathbb{M}(G)$ the (covariant) Cartier-Dieudonné module of $G$ [3, Chapitre II, Sect. 1]; we also denote $\mathbb{F}_{G}$ and $\mathbb{V}_{G}$ (or simply $\mathbb{F}$ and $\mathbb{V}$ when there is no confusion) the Frobenius and Verschiebung endomorphisms of $\mathbb{M}(G)$. If $B$ is $\mathbb{Z}_{p^{2}}$-algebra, where $\mathbb{Z}_{p^{2}}$ is the valuation ring of $\mathbb{Q}_{p^{2}}$, and $G$ a formal $\mathcal{O}_{D}$-module, then we may define

$$
\begin{aligned}
& \operatorname{Lie}^{0}(G)=\left\{m \in \operatorname{Lie}(G): \iota_{G}(a)=a m, a \in \mathbb{Z}_{p^{2}}\right\}, \\
& \operatorname{Lie}^{1}(G)=\left\{m \in \operatorname{Lie}(G): \iota_{G}(a)=\bar{a} m, a \in \mathbb{Z}_{p^{2}}\right\}
\end{aligned}
$$

and, since $G$ is special, both $\operatorname{Lie}^{0}(G)$ and $\operatorname{Lie}^{1}(G)$ are free $B$-modules of rank 1, (recall that $\bar{x}=\sigma(x)$, so $x \mapsto \bar{x}$ is the non-trivial automorphism of $\left.\operatorname{Gal}\left(\mathbb{Q}_{p^{2}} / \mathbb{Q}_{p}\right)\right)$. Moreover, $\mathbb{M}(G)$ is also equipped with a graduation $\mathbb{M}(G)=\mathbb{M}^{0}(G) \oplus \mathbb{M}^{1}(G)$ where

$$
\begin{aligned}
& \mathbb{M}^{0}(G)=\left\{m \in \mathbb{M}(G): \iota_{G}(a)=a m, a \in \mathbb{Z}_{p^{2}}\right\}, \\
& \mathbb{M}^{1}(G)=\left\{m \in \mathbb{M}(G): \iota_{G}(a)=\bar{a} m, a \in \mathbb{Z}_{p^{2}}\right\} .
\end{aligned}
$$

Fix a SFD-module $\Phi=G \times G$ over $\overline{\mathbb{F}}_{p}$, where $G$ is the reduction modulo $p$ of a LubinTate formal group $\hat{\mathcal{E}}$ of height 2 over $\hat{\mathbb{Z}}_{p}^{\text {unr }}$, the completion of the valuation ring of the maximal unramifed extension $\mathbb{Z}_{p}^{\text {unr }}$ of $\mathbb{Z}_{p}$; so $\hat{\mathcal{E}}$ is the formal group of a supersingular elliptic curve $\mathcal{E}$ over $\mathbb{Z}_{p}^{\text {unr }}$ (see [43, Definition 9 and Remark 27]). The Dieudonné module $\mathbb{M}(\Phi)$ of $\Phi$ is the $\hat{\mathbb{Z}}_{p}^{\text {unr }}[\mathbb{F}, \mathbb{V}]$-module with $\mathbb{V}$-basis $g^{0}$ and $g^{1}$, satisfying the relations $\mathbb{F}\left(g^{0}\right)=\mathbb{V}\left(g^{0}\right)$ and $\mathbb{F}\left(g^{1}\right)=\mathbb{V}\left(g^{1}\right)$. The quaternionic order $\mathcal{O}_{D}$ acts via the rules $\Pi\left(g^{0}\right)=\mathbb{V}\left(g^{1}\right), \Pi\left(g^{1}\right)=$ $\mathbb{V}\left(g^{0}\right)$ and $a\left(g^{0}\right)=a g^{0}, a\left(g^{1}\right)=\bar{a} g^{1}$ for $a \in \mathbb{Z}_{p^{2}}$. By [43, Corollary 30], $\eta^{0}(\Phi)$ is generated over $\mathbb{Z}_{p}$ by $\left[g^{0}, 0\right]$ and $\left[\mathbb{V}\left(g^{1}\right), 0\right]$, and $\eta^{1}(\Phi)$ is generated over $\mathbb{Z}_{p}$ by $\left[g^{1}, 0\right]$ and $\left[\mathbb{V}\left(g^{0}\right), 0\right]$.

Let Nilp denote the category of $\mathbb{Z}_{p}$-algebras in which $p$ is nilpotent. Denote SFD the functor on Nilp which associates to each $B \in$ Nilp the set $\operatorname{SFD}(B)$ of isomorphism classes of triples $(\psi, G, \rho)$ where

(1) $\psi: \overline{\mathbb{F}}_{p} \rightarrow B / p B$ is an homomorphism,

(2) $G$ is a SFD-module over $B$ of height 4 ,

(3) $\rho: \psi_{*} \Phi \rightarrow G_{B / p B}=G \otimes_{B} B / p B$ is a quasi-isogeny of height 0 , called rigidification. 
See [43, page 663] or [3, Chapitre II (8.3)] for more details on the definition of the functor SFD. Drinfel'd shows in [13] that the functor SFD is represented by the $\mathbb{Z}_{p}$-formal scheme

$$
\hat{\mathcal{H}}_{p}^{\text {unr }}=\hat{\mathcal{H}}_{p} \hat{\otimes}_{\mathbb{Z}_{p}} \hat{\mathbb{Z}}_{p}^{\text {unr }}
$$

(see [43, Theorem 28], [3, Chapitre II (8.4)]). Note that $\hat{\mathcal{H}}_{p}^{\text {unr }}$, considered as $\hat{\mathbb{Z}}_{p}^{\text {unr }}$-formal

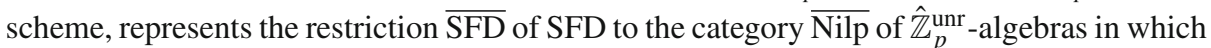
$p$ is nilpotent ( $c f$. [3, Chapitre II, Sect. 8]). Unless otherwise stated, we will see $\hat{\mathcal{H}}_{p}^{\text {unr }}$ as a $\hat{\mathbb{Z}}_{p}^{\text {unr }}$-formal scheme.

For later use, we review some of the steps involved in the proof of Drinfel'd Theorem. The crucial step is the interpretation of the $\mathbb{Z}_{p}$-formal scheme $\hat{\mathcal{H}}_{p}$ as the solution of a moduli problem. For $B \in \mathrm{Nilp}$, a compatible data on $S=\operatorname{Spf}(B)$ consists of a quadruplet $(\eta, T, u, \rho)$ where

(1) $\eta=\eta^{0} \oplus \eta^{1}$ is a sheaf of flat $\mathbb{Z} / 2 \mathbb{Z}$-graded $\mathbb{Z}_{p}[\Pi]$-modules on $S$,

(2) $T=T^{0} \oplus T^{1}$ is a $\mathbb{Z} / 2 \mathbb{Z}$-graded sheaf of $\mathcal{O}_{S}[\Pi]$-modules with $T^{i}$ invertible,

(3) $u: \eta \rightarrow T$ is a homogeneous degree zero map such that $u \otimes 1: \eta \otimes_{\mathbb{Z}_{p}} \mathcal{O}_{S} \rightarrow T$ is surjective,

(4) $\rho:\left(\mathbb{Q}_{p}^{2}\right)_{S} \rightarrow \eta_{0} \otimes_{\mathbb{Z}_{p}} \mathbb{Q}_{p}$ is a $\mathbb{Q}_{p}$-linear isomorphism,

which satisfy natural compatibilities, denoted $(\mathbf{C 1}),(\mathbf{C 2}),(\mathbf{C 3})$ in [43, page 652], to which we refer for details. The first step in Drinfel'd work is to show that the $\mathbb{Z}_{p}$-formal sheme $\hat{\mathcal{H}}_{p}$ represents the functor which associates to each $B \in$ Nilp the set of admissible quadruplets over $B$. To each compatible data $\mathcal{D}=(\eta, T, u, \rho)$ on $S$ one associates a $S$-valued point $\Psi: S \rightarrow \hat{\mathcal{H}}_{p}$ of $\hat{\mathcal{H}}_{p}$, as explained in [43, pages 652-655]. The second step to prove the representability of $\overline{\mathrm{SFD}}$ is to associate with any $B \in \overline{\operatorname{Nilp}}$ and $X=(\psi, G, \rho) \in \operatorname{SFD}(B)$ a quadruplet $\left(\eta_{X}, T_{X}, u_{X}, \rho_{X}\right)$ which corresponds to an $S=\operatorname{Spf}(B)$-valued point on $\hat{\mathcal{H}}_{p} \hat{\otimes}_{\mathbb{Z}_{p}} \hat{\mathbb{Z}}_{p}^{\text {unr }}$. If $X=(\psi, G, \rho) \in \operatorname{SFD}(B)$ is given as above, the quadruplet $\left(\eta_{X}, T_{X}, u_{X}, \rho_{X}\right)$ can be explicitly constructed as follows:

- $T_{X}=T(G)=\mathbb{M}(G) / \mathbb{V M}(G)$ the tangent space to $G$ at the origin, equipped with its graduation defined previously;

- Define $\mathbb{N}(G)=\mathbb{M}(G) \times \mathbb{M}(G) / \sim$ where $(\mathbb{V}(x), 0) \sim(0, \Pi(x))$; we denote $[x, y]$ the class in $\mathbb{N}$ represented by the pair $(x, y)$. Let $\lambda: \mathbb{N}(G) \rightarrow \mathbb{M}(G)$ be the map defined by $\lambda([x, y])=\Pi(x)-\mathbb{V}(y)$. There is a map $\mathbb{N}(G) \rightarrow \mathbb{M}(G) / \mathbb{V} \mathbb{M}(G)$ induced by the projection onto the first component. Further, one easily shows that there exists a unique map $L: \mathbb{M}(G) \rightarrow \mathbb{N}(G)$ satisfying the relation $\lambda \circ L=\mathbb{F}$. Let $\phi: \mathbb{N}(G) \rightarrow \mathbb{N}(G)$ be defined by $\phi([x, y])=L(x)+[y, 0]$. Then $\eta_{X}=\eta(G)=\mathbb{N}^{\phi=I d}$. The graduation of $\mathbb{M}(G)$ defines a graduation $\eta(G)=\eta^{0}(G) \oplus \eta^{1}(G)$.

- $u_{X}=u(G)$ is induced by the projection map $\mathbb{N}(G) \rightarrow \mathbb{M}(G) / \mathbb{V M}(G)$.

- Fix an isomorphism $\eta^{0}(\Phi) \simeq \mathbb{Z}_{p} \oplus \mathbb{Z}_{p}$. The quasi-isogeny $\rho$ induces a map

$$
\rho_{X}=\rho(G): \mathbb{Q}_{p} \oplus \mathbb{Q}_{p} \simeq \eta^{0}\left(\psi_{*}(\Phi)\right) \otimes_{\mathbb{Z}_{p}} \mathbb{Q}_{p} \rightarrow \eta^{0}(\Phi) \otimes_{\mathbb{Z}_{p}} \mathbb{Q}_{p}
$$

We finally discuss rigid analytic parameters [43]. With an abuse of notation, let SFD be the functor from the category pro-Nilp of projective limits of objects in Nilp associated with SFD. In [43, Def. 10], Teitelbaum introduces a function

$$
z_{0}: \operatorname{SFD}\left(\hat{\mathbb{Z}}_{p}^{\text {unr }}\right) \longrightarrow \mathcal{H}_{p}\left(\hat{\mathbb{Q}}_{p}^{\text {unr }}\right)
$$

such that the map $X=(\psi, G, \rho) \mapsto\left(z_{0}(X), \psi\right)$ gives a bijection between $\operatorname{SFD}\left(\hat{\mathbb{Z}}_{p}^{\text {unr }}\right)$ and $\left(\hat{\mathcal{H}}_{p} \hat{\otimes}_{\mathbb{Z}_{p}} \hat{\mathbb{Z}}_{p}^{\text {unr }}\right)\left(\hat{\mathbb{Z}}_{p}^{\text {unr }}\right)$, which we identify with the set $\mathcal{H}_{p}\left(\hat{\mathbb{Q}}_{p}^{\text {unr }}\right) \times \operatorname{Hom}\left(\hat{\mathbb{Z}}_{p}^{\text {unr }}, \hat{\mathbb{Z}}_{p}^{\text {unr }}\right)$. We call 
the map $X \mapsto z_{0}(X)$ a rigid analytic parameter on SFD. If we let pro-Nilp the category of projective limits of objects in $\overline{\mathrm{Nilp}}$, and we still denote $\overline{\mathrm{SFD}}$ the restriction of SFD to $\overline{\text { pro-Nilp, }}$ this implies that the map $X=(\psi, G, \rho) \mapsto z_{0}(X)$ gives a bijection between $\overline{\operatorname{SFD}}\left(\hat{\mathbb{Z}}_{p}^{\text {unr }}\right)$ and $\mathcal{H}_{p}\left(\hat{\mathbb{Q}}_{p}^{\text {unr }}\right)$. By [43, Thm. 45], for each $z \in \mathcal{H}_{p}\left(\hat{\mathbb{Q}}_{p}^{\text {unr }}\right)$, there exists triple $X=(\psi, G, \rho)$ in $\overline{\operatorname{SFD}}\left(\hat{\mathbb{Z}}_{p}^{\text {unr }}\right)$ such that $z_{0}(X)=z$.

\subsection{Filtered Frobenius modules}

Let $E$ be an unramified field extension of $\mathbb{Q}_{p}$. A Frobenius module $\mathbb{E}$ over $E$ is a pair $\mathbb{E}=(V, \phi)$ consisting of a finite dimensional $E$-vector space $V$ with a $\sigma$-linear isomorphism $\phi$, called Frobenius [44, Chapter VI, §1]; we also call $\phi$-modules these objects. A filtered Frobenius module is a Frobenius module $(V, \phi)$ equipped with an exhaustive and separate filtration $F^{\bullet} V$; we also call filtered $\phi$ - modules these objects.

If $G$ is a $p$-divisible formal group over $\overline{\mathbb{F}}_{p}$, one can define its first crystalline cohomology cohomology group as in [6,16], [2, Définition 2.5.7], in terms of the crystalline Dieudonné functor (among many other references, see for example [10, 12, 21] for selfcontained expositions). In the following we will denote $H_{\text {cris }}^{1}(G)$ the global sections of the crystalline Dieudonné functor (defined as in [2, Théorème 4.2.8.1]) tensored over $\hat{\mathbb{Z}}_{p}^{\text {unr }}$ with $\hat{\mathbb{Q}}_{p}^{u n r}$. By construction, $H_{\text {cris }}^{1}(G)$ is then a Frobenius module. Moreover, the canonical isomorphism between $H_{\text {cris }}^{1}(G)$ and the first de Rham cohomology group $H_{\mathrm{dR}}^{1}(G)$ of $G$ equips $H_{\text {cris }}^{1}(G)$ with a canonical filtration (arising from the Hodge filtration in the de Rham cohomology), making $H_{\mathrm{dR}}^{1}(G)$ a filtered Frobenius module; see [38].

Let $G$ be a SFD-module over $\overline{\mathbb{F}}_{p}$. Then the Frobenius module $H_{\text {cris }}^{1}(G)$ is a fourdimensional $\hat{\mathbb{Q}}_{p}^{\text {unr }}$-vector space, equipped with its $\sigma$-linear Frobenius $\phi_{\text {cris }}(G)$. It is also equipped with a $D$-module structure $j_{G}: D \hookrightarrow \operatorname{End}_{\hat{\mathbb{Q}}_{p}^{\text {unr }}}\left(H_{\text {cris }}^{1}(G)\right)$ which commutes with $\phi_{\text {cris }}(G)$, and a $\mathbb{Q}_{p}$-algebra embedding $i_{G}: \mathrm{M}_{2}\left(\mathbb{Q}_{p}\right) \hookrightarrow \operatorname{End}_{\hat{\mathbb{Q}}_{p}^{\text {unr }}}\left(H_{\text {cris }}^{1}(G)\right)$ induced by the isomorphism $\operatorname{End}_{\mathcal{O}_{D}}(G) \simeq \mathrm{M}_{2}\left(\mathbb{Q}_{p}\right)$, which commutes with the $D$-action. Define $\phi_{\text {cris }}^{\prime}(G)=j_{G}(\Pi)^{-1} \phi_{\text {cris }}(G)$ and put

$$
V_{\text {cris }}(G)=H_{\text {cris }}^{1}(G)^{\phi_{\text {cris }}^{\prime}(G)=\mathrm{Id}} .
$$

Denote $\phi_{V_{\text {cris }}(G)}=j_{G}(\Pi)_{\mid V_{\text {cris }}(G)}$ the restriction of $j_{G}(\Pi)$ to $V_{\text {cris }}(G)$. Moreover, denote

$$
\left(\eta^{i}(G) \otimes_{\mathbb{Z}_{p}} \mathbb{Q}_{p}\right)^{\vee}=\operatorname{Hom}_{\mathbb{Q}_{p}}\left(\eta^{i}(G) \otimes_{\mathbb{Z}_{p}} \mathbb{Q}_{p}, \mathbb{Q}_{p}\right)
$$

the $\mathbb{Q}_{p}$-linear dual of $\eta^{i}(G) \otimes_{\mathbb{Z}_{p}} \mathbb{Q}_{p}$.

The following lemma is crucial in what follows, and identifies $V_{\text {cris }}(G)$ with $\left(\eta^{i}(G) \otimes_{\mathbb{Z}_{p}}\right.$ $\left.\mathbb{Q}_{p}\right)^{\vee}$, from which one deduces a complete description of the filtered Frobenius module $H_{\text {cris }}^{1}(G)$. It appears in a slightly different version in the proof of [23, Lemma 5.10]. Since we did not find an reference for this fact in the form we need it, we add a complete proof.

Lemma 4.1 There is a canonical isomorphism $V_{\text {cris }}(G) \simeq\left(\eta(G) \otimes_{\mathbb{Z}_{p}} \mathbb{Q}_{p}\right)^{\vee}$ of $\mathbb{Q}_{p}$-vector spaces. Moreover, $H_{\text {cris }}^{1}(G)=V_{\text {cris }}(G) \otimes \mathbb{Q}_{p} \hat{\mathbb{Q}}_{p}^{\text {unr }}$, where the right hand side is equipped with the structure of $\hat{\mathbb{Q}}_{p}^{\mathrm{unr}}$-vector space given by $x \cdot(v \otimes \alpha)=v \otimes(\sigma(x) \alpha)$ for $v \in V_{\text {cris }}(G), x, \alpha \in$ $\hat{\mathbb{Q}}_{p}^{\mathrm{unr}}$. Finally, under this isomorphism the Frobenius $\phi_{\text {cris }}(G)$ corresponds to $\phi_{V_{\text {cris }}(G)} \otimes \sigma$. 
Proof Recall that the Frobenius module $H_{\text {cris }}^{1}(G)$ is canonically isomorphic to the contravariant Dieudonné module of $G$ with $p$ inverted, and with $\hat{\mathbb{Q}}_{p}^{\text {unr }}$-action twisted by the Frobenius automorphism $\sigma$ of $\hat{\mathbb{Q}}_{p}^{\text {unr }}$, equipped with the canonical Frobenius of the contravariant Dieudonné module (see [2, 4.2.14]). More precisely, denote

$$
\mathbb{D}(G)=\operatorname{Hom}_{\hat{\mathbb{Q}}_{p}^{\text {unr }}}\left(\mathbb{M}(G)[1 / p], \hat{\mathbb{Q}}_{p}^{\text {unr }}\right)
$$

the $\widehat{\mathbb{Q}}_{p}^{\text {unr }}$-linear dual of the covariant Dieudonné module $\mathbb{M}(G)$ of $G$ with $p$ inverted, and let

$$
\mathbb{D}(G)^{\sigma}=\mathbb{D}(G) \otimes_{\hat{\mathbb{Q}}_{p}^{\text {unr }}, \sigma} \hat{\mathbb{Q}}_{p}^{\text {unr }},
$$

where the tensor product is taken with respect to the Frobenius endomorphism $\sigma$ of $\hat{\mathbb{Q}}_{p}^{\text {unr }}$. Then as $\hat{\mathbb{Q}}_{p}^{\text {unr }}$-vector spaces, we have $H_{\text {cris }}^{1}(G) \simeq \mathbb{D}(G)^{\sigma}$. Under this isomorphism the Frobenius $\phi_{\text {cris }}(G)$ is given by the map $\varphi \mapsto \sigma \circ \varphi \circ \mathbb{V}_{G}$ for $\varphi \in \mathbb{D}(G)$.

Now, by [3, Lemme (5.12)], we have an isomorphism of $\sigma^{-1}$-isocrystals

$$
\left(\mathbb{M}^{i}(G)[1 / p], \mathbb{V}_{G} \Pi^{-1}\right) \simeq\left(\eta^{i}(G) \otimes_{\mathbb{Z}_{p}} \hat{\mathbb{Q}}_{p}^{\mathrm{unr}}, \sigma^{-1}\right)
$$

for each index $i=0,1$ (where the action of $\sigma^{-1}$ on $\eta^{i}(G) \otimes_{\mathbb{Z}_{p}} \hat{\mathbb{Q}}_{p}^{\text {unr }}$ is on the second factor only). We may therefore compute $V_{\text {cris }}(G)$ in terms of the isocrystal $\left(\eta^{i}(G) \otimes_{\mathbb{Z}_{p}} \hat{\mathbb{Q}}_{p}^{\text {unr }}, \sigma^{-1}\right)$.

As above, define

$$
\mathbb{D}^{i}(G)=\operatorname{Hom}_{\hat{\mathbb{Q}}_{p}^{\text {unr }}}\left(\mathbb{M}^{i}(G)[1 / p], \hat{\mathbb{Q}}_{p}^{\text {unr }}\right)
$$

$\left(\hat{\mathbb{Q}}_{p}^{\text {unr }}\right.$-linear dual) and let $\mathbb{D}^{i}(G)^{\sigma}$ denote the base change $\mathbb{D}^{i}(G) \otimes_{\hat{\mathbb{Q}}_{p}^{\text {unr }}, \sigma} \hat{\mathbb{Q}}_{p}^{\text {unr }}$ via $\sigma$. Since $\mathbb{M}(G)=\mathbb{M}^{0}(G) \oplus \mathbb{M}^{1}(G)$, we have

$$
\mathbb{D}(G)^{\sigma}=\mathbb{D}^{0}(G)^{\sigma} \oplus \mathbb{D}^{1}(G)^{\sigma},
$$

and we may write any element $\varphi \in \mathbb{D}(G)^{\sigma}$ as a pair $\left(\varphi_{0}, \varphi_{1}\right)$ with $\varphi_{i} \in \mathbb{D}^{i}(G)^{\sigma}, i=$ 0,1 . By definition, an element $\varphi=\left(\varphi_{0}, \varphi_{1}\right) \in \mathbb{D}(G)^{\sigma}$ belongs to $V_{\text {cris }}(G)$ if and only if $\varphi_{i}\left(V_{G} \Pi^{-1}\left(m_{i}\right)\right)$ is equal to $\sigma^{-1}\left(\varphi_{i}\left(m_{i}\right)\right)$ for all $m_{i} \in \mathbb{M}^{i}(G)[1 / p]$, and for all $i=0,1$. Using (14), identify $\varphi_{i}$ with a $\hat{\mathbb{Q}}_{p}^{\text {unr }}$-linear homomorphism $\varphi_{i}: \eta^{i}(G) \otimes_{\mathbb{Z}_{p}} \hat{\mathbb{Q}}_{p}^{\text {unr }} \rightarrow \hat{\mathbb{Q}}_{p}^{\text {unr }}$, denoted with a slight abuse of notation with the same symbol; then the above equation describing $V_{\text {cris }}(G)$ becomes

$$
\varphi_{i}\left(n \otimes \sigma^{-1}(x)\right)=\sigma^{-1}\left(\varphi_{i}(n \otimes x)\right)
$$

for all $n \in \eta^{i}(G)$ and all $x \in \hat{\mathbb{Q}}_{p}^{\text {unr }}$. Since $\varphi_{i}$ is $\hat{\mathbb{Q}}_{p}^{\text {unr }}$-linear,

$$
\sigma^{-1}\left(\varphi_{i}(n \otimes x)\right)=\sigma^{-1}(x) \sigma^{-1}\left(\varphi_{i}(n \otimes 1)\right),
$$

and we deduce the equality $\varphi_{i}\left(n \otimes \sigma^{-1}(x)\right)=\sigma^{-1}(x) \sigma^{-1}\left(\varphi_{i}(n \otimes 1)\right)$ for all $n \in \eta^{i}(G)$. Taking $x=1$ we see that $\varphi_{i}(n \otimes 1)=\sigma^{-1}\left(\varphi_{i}(n \otimes 1)\right)$ and we conclude that $\varphi_{i}(n \otimes 1) \in \mathbb{Q}_{p}$ for all $n \in \eta^{i}(G)$. So $\varphi_{i}$ is the $\hat{\mathbb{Q}}_{p}^{\text {unr }}$-linear extension of a $\mathbb{Q}_{p}$-linear homomorphism

$$
\eta^{i}(G) \otimes_{\mathbb{Z}_{p}} \mathbb{Q}_{p} \rightarrow \mathbb{Q}_{p} .
$$

Since $\eta(G)=\eta^{0}(G) \oplus \eta^{1}(G)$, we then conclude that $V_{\text {cris }}(G) \simeq\left(\eta(G) \otimes_{\mathbb{Z}_{p}} \mathbb{Q}_{p}\right)^{\vee}$ as $\mathbb{Q}_{p^{-}}$ vector spaces (here ${ }^{\vee}$ denotes the $\mathbb{Q}_{p}$-dual). If $n_{1}, \ldots, n_{4}$ is a $\mathbb{Q}_{p}$-basis of $\eta(G) \otimes_{\mathbb{Z}_{p}} \mathbb{Q}_{p}$, then $d n_{1}, \ldots, d n_{4}$ defined by $d n_{i}\left(n_{j}\right)=\delta_{i, j}$ (as usual, $\delta_{i, j}=1$ if $i=j$ and 0 otherwise) is 
a basis of $\left(\eta(G) \otimes_{\mathbb{Z}_{p}} \mathbb{Q}_{p}\right)^{\vee}$ and, by $\hat{\mathbb{Q}}_{p}^{\text {unr }}$-linear extension, also of $\left(\eta(G) \otimes_{\mathbb{Z}_{p}} \hat{\mathbb{Q}}_{p}^{\text {unr }}\right)^{\vee}$. If we now base change the $\hat{\mathbb{Q}}_{p}^{\text {unr }}$-vector space $\left(\eta(G) \otimes_{\mathbb{Z}_{p}} \hat{\mathbb{Q}}_{p}^{\text {unr }}\right)^{\vee}$ via $\sigma$, we see that $d n_{1}, \ldots, d n_{4}$ is still a $\hat{\mathbb{Q}}_{p}^{\text {unr }}$-basis, and we have

$$
\left(x \cdot d n_{i}\right)\left(n_{j}\right)=\sigma(x) \delta_{i, j}
$$

for all $x \in \hat{\mathbb{Q}}_{p}^{\text {unr }}$. Using the above description of $H_{\text {cris }}^{1}(G)$ in terms of $\mathbb{D}(G)^{\sigma}$, and the description of $V_{\text {cris }}(G)$ in terms of $\eta(G)$, we have an isomorphism of $\hat{\mathbb{Q}}_{p}^{\text {unr }}$-vector spaces,

$$
H_{\text {cris }}^{1}(G) \simeq\left(V_{\text {cris }}(G) \otimes_{\mathbb{Q}_{p}} \hat{\mathbb{Q}}_{p}^{\text {unr }}\right)^{\sigma}
$$

where the upper index $\sigma$ on the right hand side means that the structure of $\hat{\mathbb{Q}}_{p}^{\text {unr }}$-vector space is twisted by $\sigma$ as explained above. Moreover, the $\sigma^{-1}$-linear isomorphism $\mathbb{V}_{G} \Pi^{-1}$ of $\mathbb{M}(G)[1 / p]$ corresponds to the $\sigma^{-1}$-linear isomorphism $\sigma^{-1}$ of $\eta(G) \otimes_{\mathbb{Z}_{p}} \hat{\mathbb{Q}}_{p}^{\text {unr }}$ (acting on the second component only), and therefore the isomorphism $\varphi \mapsto \sigma \circ \varphi \circ \mathbb{V}_{G}$ of $\mathbb{M}(G)[1 / p]^{\vee}$ (where ${ }^{\vee}$ denotes the $\hat{\mathbb{Q}}_{p}^{\text {unr }}$-linear dual) corresponds to the isomorphism $\Pi \otimes \sigma$ of $\left(\left(\eta(G) \otimes_{\mathbb{Z}_{p}} \mathbb{Q}_{p}\right)^{\vee} \otimes_{\mathbb{Q}_{p}} \hat{\mathbb{Q}}_{p}^{\text {unr }}\right)^{\sigma}$ given by $d n_{i} \otimes x \mapsto\left(d n_{i} \circ \Pi\right) \otimes \sigma(x)$ where $\left(d n_{i} \circ \Pi\right)(n)=n_{i}(\Pi n)$, which corresponds to $\phi_{V_{\text {cris }}(G)} \otimes \sigma$ by definition of $\phi_{V_{\text {cris }}(G)}$.

\subsection{Filtered convergent $F$-isocrystals}

To describe the relative de Rham cohomology of the $p$-adic upper half plane, we first need some preliminaries on the notion of filtered convergent $F$-isocrystals introduced in [23]. Let $E \subseteq \mathbb{Q}_{p}^{\text {unr }}$ be an unramified extension of $\mathbb{Q}_{p}$, with valuation ring $\mathcal{O}_{E}$. If $\left(X, \mathcal{O}_{X}\right)$ is a $p$-adic $\mathcal{O}_{E}$-formal scheme, we denote $\left(X^{\text {rig }}, \mathcal{O}_{X}^{\text {rig }}\right)$ the associated $E$-rigid analytic space (or its generic fiber), and if $\mathcal{F}$ is a sheaf of $\mathcal{O}_{X}$-modules, we denote $\mathcal{F}^{\text {rig }}$ its associated sheaf of $\mathcal{O}_{X}^{\text {rig }}$-modules [8, Sect. 7.4], [5, Sect. 1].

The notion of convergent isocrystal on a $p$-adic, formally smooth $\mathcal{O}_{E}$-formal scheme $X$ is introduced in [23, Definition 3.1], and we refer to loc. cit. for details; we only recall that a convergent isocrystal on $X$ is a rule $\mathcal{E}$ which assigns to each enlargement $\left(T, z_{T}\right)$ of $X$ a coherent $\mathcal{O}_{T} \otimes_{\mathcal{O}_{E}} E$-module $\mathcal{E}_{T}$ satisfying a natural cocycle condition for morphism of enlargements. Also recall from [23, Definition 3.2] that a convergent $F$-isocrystal on $X$ is a convergent isocrystal $\mathcal{E}$ on $X$ equipped with an isomorphism of convergent isocrystals $\phi_{\mathcal{E}}: F^{*} \mathcal{E} \simeq \mathcal{E}$; here $F$ is the absolute Frobenius of the reduced closed subscheme of the closed subscheme of $X$ defined by the ideal $p \mathcal{O}_{X}$. By [39, 1.20, 2.81] is a canonical integrable connection $\nabla_{X}: \mathcal{E}_{X} \rightarrow \mathcal{E}_{X}^{\text {rig }} \otimes \Omega_{X^{\text {rig }}}^{1}$. Accordingly with [23, Definition 3.3], a filtered convergent $F$-isocrystal on $X$ is a $F$-isocrystal $\left(\mathcal{E}, \phi_{\mathcal{E}}\right)$ such that $\mathcal{E}_{X}^{\text {rig }}$ is equipped with an exhaustive and separated decreasing filtration $F^{\bullet} \mathcal{E}_{X}^{\text {rig }}$ of coherent $\mathcal{O}_{X}^{\text {rig }}$-submodules such that $\nabla_{X}^{\text {rig }}\left(\mathcal{F}^{i} \mathcal{E}_{X}^{\text {rig }}\right)$ is contained in $\mathcal{F}^{i-1} \mathcal{E}_{X}^{\text {rig }} \otimes_{\mathcal{O}_{X}^{\text {rig }}} \Omega_{X^{\text {rig }}}^{1}$ for all $i$; in these definitions, $F$ is the absolute Frobenius of the reduced closed subscheme of the closed subscheme of $X$ defined by the ideal $p \mathcal{O}_{X}$.

We denote $\mathcal{E}\left(\mathcal{O}_{X}\right)$ the identity object of the additive tensor category of convergent filtered $F$-isocrystals on $X$, introduced in [23, Example 3.4(a)] and defined on enlargements by the rule $\left(T, z_{T}\right) \rightsquigarrow \mathcal{O}_{T} \otimes_{\mathcal{O}_{E}} E$; it is equipped with canonical Frobenius and filtration (see loc. cit. for details). We also denote $\mathcal{E}(V)=V \otimes_{\mathbb{Q}_{p}} \mathcal{E}\left(\mathcal{O}_{\hat{\mathcal{H}}_{p}^{\text {unr }}}\right)$ the filtered convergent $F$-isocrystal attached to a $\mathbb{Q}_{p}$-rational, finite dimensional representation $\rho: \mathrm{GL}_{2} \times \mathrm{GL}_{2} \rightarrow \mathrm{GL}(V)$ of 
the algebraic group $\mathrm{GL}_{2} \times \mathrm{GL}_{2}$; see [23, pages 345-346] for the definition of Frobenius and filtration.

The following more articulated example of filtered convergent $F$-isocrystal arises from relative de Rham cohomology of the universal SFD-module. We follow closely [14, 23]. Let $\left(\lambda_{\mathcal{G}}, \mathcal{G}, \rho_{\mathcal{G}}\right)$ be the universal triple, arising from the representability of the functor SFD by $\hat{\mathcal{H}}_{p}^{\text {unr }}$; denote $\lambda: \mathcal{G} \rightarrow \hat{\mathcal{H}}_{p}^{\text {unr }}$ be universal map. Then

$$
\mathcal{E}(\mathcal{G})=\mathbb{R}^{1} \lambda_{*}\left(\mathcal{O}_{\mathcal{G} / \hat{\mathbb{Q}}_{p}^{\text {unr }}}\right)
$$

has a structure of convergent $F$-isocrystal of $\hat{\mathcal{H}}_{p}^{\text {unr }}$, which interpolates crystalline cohomology sheaves (see [39, Theorem (3.1), Theorem (3.7)]; see also [6, 7]). The coherent $\mathcal{O}_{\mathcal{H}_{p}^{\text {unr }}}$-module $\mathcal{E}(\mathcal{G})_{\hat{\mathcal{H}}_{p}^{\text {unr }}}^{\text {rig }}$ is canonically isomorphic to the relative rigid de Rham cohomology sheaf

$$
\mathcal{H}_{\mathrm{dR}}^{1}\left(\mathcal{G}^{\text {rig }} / \mathcal{H}_{p}^{\text {unr }}\right)=\mathbb{R}^{1} \lambda_{*}^{\text {rig }}\left(\Omega_{\mathcal{G}^{\text {rig }} / \mathcal{H}_{p}^{\text {unr }}}\right)
$$

The canonical integrable connection $\nabla_{\hat{\mathcal{H}}_{p}^{\text {unr }}}^{\text {rig }}$ coincides with the Gauss-Manin connection

$$
\nabla_{\mathcal{G}}^{\text {rig }}: \mathcal{H}_{\mathrm{dR}}^{1}\left(\mathcal{G}^{\text {rig }} / \mathcal{H}_{p}^{\text {unr }}\right) \longrightarrow \Omega_{\mathcal{H}_{p}^{\text {unr }} / \hat{\mathbb{Q}}_{p}^{\text {unr }}} \otimes_{\mathcal{O}_{\mathcal{H}_{p}^{\text {unr }}}} \mathcal{H}_{\mathrm{dR}}^{1}\left(\mathcal{G}^{\text {rig }} / \mathcal{H}_{p}^{\text {unr }}\right)
$$

whose construction in this context follows [27]; see [23, Example 3.4(c)], [39, Theorem (3.10)]. The Hodge filtration on the de Rham cohomology $\mathcal{H}_{\mathrm{dR}}^{1}\left(\mathcal{G}^{\text {rig }} / \mathcal{H}_{p}^{\text {unr }}\right)$ makes then $\mathcal{E}(\mathcal{G})$ a filtered convergent $F$-isocrystal. To describe this filtration more explicitly, denote

$$
\mathcal{H}_{\mathrm{dR}}^{1}(\mathcal{G})=\mathcal{H}_{\mathrm{dR}}^{1}\left(\mathcal{G} / \hat{\mathcal{H}}_{p}^{\mathrm{unr}}\right)
$$

the dual of the Lie algebra of the universal vectorial extension of $\mathcal{G}$, equipped with its structure of convergent $F$-isocrystal ([35, Chapter IV, §2], [36, Sect. 1,9,11)]). By [2, Sect. 3.3], we have an isomorphism of convergent $F$-isocrystals

$$
\mathcal{E}(\mathcal{G}) \simeq \mathcal{H}_{\mathrm{dR}}^{1}(\mathcal{G})
$$

such that the Hodge filtration on the de Rham cohomology groups coincides with the HodgeTate filtration

$$
0 \longrightarrow \underline{\omega}_{\mathcal{G}}^{\text {rig }} \longrightarrow \mathcal{H}_{\mathrm{dR}}^{1, \text { rig }}(\mathcal{G}) \longrightarrow \mathcal{L} i e_{\mathcal{G}^{\vee}}^{\text {rig }} \longrightarrow 0
$$

Here $\underline{\omega}_{\mathcal{G}}=e_{\mathcal{G}}^{*}\left(\Omega_{\mathcal{G} / \hat{\mathcal{H}}_{p}^{\text {unr }}}^{1}\right)$, where $e_{\mathcal{G}}: \hat{\mathcal{H}}_{p}^{\text {unr }} \rightarrow \mathcal{G}$ is the zero-section, $\mathcal{H}_{\mathrm{dR}}^{1, \text { rig }}(\mathcal{G})=$ $\mathcal{H}_{\mathrm{dR}}^{1}(\mathcal{G})_{\hat{\mathcal{H}}_{p}^{\text {unr }}}^{\text {rig }}$, and $\mathcal{L} i e_{\mathcal{G}^{\vee}}$ is the Lie algebra of the Cartier dual $\mathcal{G}^{\vee}$ of $\mathcal{G}$. A result of Faltings [14, Sect. 5] (see also [23, Lemma 5.10]) shows that, as filtered convergent $F$-isocrystals, we have

$$
\mathcal{H}_{\mathrm{dR}}^{1}(\mathcal{G}) \simeq V_{\text {cris }}(\Phi) \otimes_{\mathbb{Q}_{p}} \mathcal{E}\left(\mathcal{O}_{\hat{\mathcal{H}}_{p}^{\mathrm{unr}}}\right) \simeq \mathcal{E}\left(\mathrm{M}_{2}\right)
$$

where $\rho: \mathrm{GL}_{2} \times \mathrm{GL}_{2} \rightarrow \mathrm{GL}\left(\mathrm{M}_{2}\right)$ is the representation defined by $\rho_{1}(A)(B)=A B$ and $\rho_{2}(A) B=B \bar{A}$ (here if $A=\left(\begin{array}{ll}a & b \\ c & d\end{array}\right)$, then $\bar{A}=\left(\begin{array}{cc}d & -b \\ -c & a\end{array}\right)$ ). 


\subsection{The Kodaira-Spencer map}

The Kodaira-Spencer map for $\lambda: \mathcal{G} \rightarrow \hat{\mathcal{H}}_{p}^{\text {unr }}$, where as above $\mathcal{G}$ is the universal SFD-module, is the composition

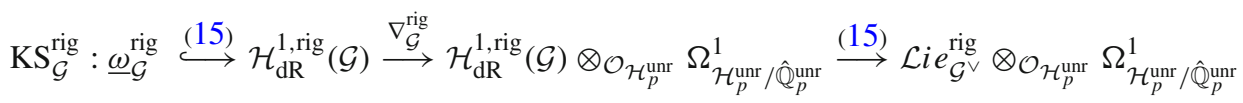

in which the first and the last map come from the Hodge-Tate exact sequence (15). Recalling the duality between $\underline{\omega}_{\mathcal{G}}$ and $\mathcal{L} i e_{\mathcal{G}}$, we therefore obtain, as in the algebraic case, a symmetric

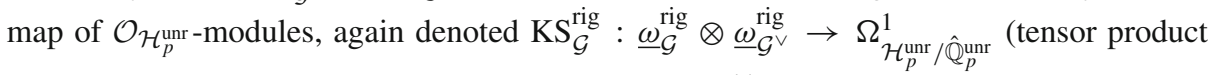
over $\mathcal{O}_{\mathcal{H}_{p}^{\text {unr }}}$. By fixing a formal polarization $\iota_{\mathcal{G}}: \mathcal{G} \simeq \mathcal{G}^{\vee}$ of $\mathcal{G}$ [3, Chapitre III, Lemma 4.4], we obtain isomorphism $\underline{\omega}_{\mathcal{G}} \simeq \omega_{\mathcal{G}} \vee$ of $\mathcal{O}_{\mathcal{H}_{p}^{\text {unr }}}$-modules, and the Kodaira-Spencer map takes the form

$$
\mathrm{KS} \mathrm{G}_{\mathcal{G}}^{\text {rig }}:\left(\underline{\omega}_{\mathcal{G}}^{\text {rig }}\right)^{\otimes 2} \longrightarrow \Omega_{\mathcal{H}_{p}^{\text {unr }} / \hat{\mathbb{Q}}_{p}^{\text {unr }}}^{1}
$$

where the tensor product is again over $\mathcal{O}_{\mathcal{H}_{p}^{\text {unr }}}$.

We now describe the Kodaira-Spencer map more explicitly, mimicking, in the complex case, $[17,37]$. Denote

$$
\mathcal{H}_{1, \mathrm{dR}}(\mathcal{G})=\mathcal{H}_{1, \mathrm{dR}}\left(\mathcal{G} / \mathcal{H}_{p}^{\mathrm{unr}}\right)
$$

the universal vectorial extension of $\mathcal{G}$, which is equipped with a structure of filtered convergent $F$-isocrystal as before; see [14, Sect. 5]. Put $\mathcal{H}_{1, \mathrm{dR}}^{\text {rig }}(\mathcal{G})=\mathcal{H}_{1, \mathrm{dR}}(\mathcal{G})_{\hat{\mathcal{H}}_{p}^{\text {unr }}}^{\text {rig }}$. By definition, the universal vectorial extensions of $\mathcal{G}$ and $\mathcal{G}^{\vee}$ are dual to each other. We therefore obtain a $\mathcal{O}_{\mathcal{H}_{p}^{\text {rig }}}$-bilinear skew-symmetric map

$$
\mathcal{H}_{\mathrm{dR}}^{1, \text { rig }}(\mathcal{G}) \times \mathcal{H}_{1, \mathrm{dR}}^{\text {rig }}(\mathcal{G}) \longrightarrow \mathcal{O}_{\mathcal{H}_{p}^{\text {unr }}} .
$$

The principal polarization $\iota_{\mathcal{G}}: \mathcal{G} \simeq \mathcal{G}^{\vee}$ identifies canonically $\mathcal{H}_{\mathrm{dR}}^{1 \text {,rig }}(\mathcal{G})$ and $\mathcal{H}_{1, \mathrm{dR}}^{\text {rig }}(\mathcal{G})$, and we therefore obtain a pairing

$$
\langle,\rangle_{\mathrm{dR}}^{\mathrm{rig}}: \mathcal{H}_{\mathrm{dR}}^{1, \text { rig }}(\mathcal{G}) \times \mathcal{H}_{\mathrm{dR}}^{1, \text { rig }}(\mathcal{G}) \longrightarrow \mathcal{O}_{\mathcal{H}_{p}^{\text {unr }}}
$$

satisfying $\langle d x, y\rangle_{\mathcal{G}}^{\text {rig }}=\left\langle x, d^{\dagger} y\right\rangle_{\mathcal{G}}^{\text {rig }}$ for all $x, y$ sections in $\mathcal{H}_{\mathrm{dR}}^{1 \text {,rig }}(\mathcal{G})$ and all $d \in D$ (because $\left.\iota_{\mathcal{G}}(d y)=d^{\dagger} \iota_{\mathcal{G}}(y)\right)$ which we call rigid polarization pairing. We may therefore construct a map

$$
\rho: \mathcal{H}_{\mathrm{dR}}^{1, \text { rig }}(\mathcal{G}) \longrightarrow\left(\mathcal{H}_{\mathrm{dR}}^{1, \text { rig }}(\mathcal{G})\right)^{\vee} \longrightarrow\left(\underline{\omega}_{\mathcal{G}}^{\text {rig }}\right)^{\vee}
$$

where the first map takes a section $s$ to the map defined for a section $t$ by $t \mapsto\langle s, t\rangle_{\mathrm{dR}}^{\mathrm{rig}}$ and the second map is induced by duality from the inclusion $\underline{\omega}_{\mathcal{G}}^{\text {rig }} \hookrightarrow \mathcal{H}_{\mathrm{dR}}^{1 \text {,rig }}(\mathcal{G})$. Fix now a section $s \in H^{0}\left(U,\left(\Omega_{\mathcal{H}_{p}^{\text {unr }} / \hat{\mathbb{Q}}_{p}^{\text {unr }}}\right)^{\vee}\right)$ over some affinoid $U$. Then we may compose the maps to get

$$
\begin{aligned}
\rho_{s}: H^{0}\left(U, \underline{\omega}_{\mathcal{G}}^{\text {rig }}\right) \longrightarrow & H^{0}\left(U, \mathcal{H}_{\mathrm{dR}}^{1, \text { rig }}(\mathcal{G})\right) \stackrel{\nabla_{\mathcal{G}}^{\text {rig }}}{\longrightarrow} H^{0}\left(U, \mathcal{H}_{\mathrm{dR}}^{1, \text { rig }}(\mathcal{G}) \otimes \Omega_{\mathcal{H}_{p}^{\text {unr }} / \hat{\mathbb{Q}}_{p}^{\text {unr }}}^{1}\right) \stackrel{1 \otimes s}{\longrightarrow} \\
& \stackrel{1 \otimes s}{\longrightarrow} H^{0}\left(U, \mathcal{H}_{\mathrm{dR}}^{1, \text { rig }}(\mathcal{G})\right) \stackrel{\rho}{\longrightarrow} H^{0}\left(U,\left(\underline{\omega}_{\mathcal{G}}^{\mathrm{rig}}\right)^{\vee}\right) .
\end{aligned}
$$


The association $s \mapsto \rho_{s}$ defines then a map of sheaves

$$
\left(\mathrm{KS}_{\mathcal{G}}^{\mathrm{rig}}\right)^{\vee}:\left(\Omega_{\mathcal{H}_{p}^{\text {unr }}}^{1} / \hat{\mathbb{Q}}_{p}^{\text {unr }}\right)^{\vee} \longrightarrow \operatorname{Hom}_{\mathcal{O}_{\mathcal{H}}^{\text {unr }}}\left(\underline{\omega}_{\mathcal{G}}^{\text {rig }},\left(\underline{\omega}_{\mathcal{G}}^{\text {rig }}\right)^{\vee}\right)
$$

By construction, the dual of this map is the Kodaira-Spencer map, under the canonical identification between $\operatorname{Hom}\left(\underline{\omega}_{\mathcal{G}}^{\text {rig }},\left(\underline{\omega}_{\mathcal{G}}^{\text {rig }}\right)^{\vee}\right)^{\vee}$ and $\left(\underline{\omega}_{\mathcal{G}}^{\text {rig }}\right)^{\otimes 2}$.

\subsection{Universal rigid data}

The aim of this subsection is to use the results of [43] to describe the Hodge filtration (15). For this, we need to recall the universal rigid data introduced in [43].

Let $V_{0}$ and $V_{1}$ be constant sheaves of one-dimensional $\mathbb{Q}_{p}$-vector spaces on the $\mathbb{Q}_{p}$-rigid analytic space $\mathcal{H}_{p}$ with basis $t_{0}$ and $t_{1}$ respectively. Define two invertible sheaves $T_{0}^{\text {univ }}$ and $T_{1}^{\text {univ }}$ on $\mathcal{H}_{p}$ by $T_{i}^{\text {univ }}=\mathcal{O}_{\mathcal{H}_{p}} \otimes V_{i}$ for $i=0,1$, where $\mathcal{O}_{\mathcal{H}_{p}}$ is the structural sheaf of rigid analytic functions on $\mathcal{H}_{p}$. Define $T^{\text {univ }}=T_{0}^{\text {univ }} \oplus T_{1}^{\text {univ }}$. For $i=0,1$, let $\eta_{i}^{\text {univ }}$ be the constant sheaf of two-dimensional $\mathbb{Q}_{p}$-vector spaces on $\mathcal{H}_{p}$ with basis $e_{i, 0}$ and $e_{i, 1}$. One fixes

$$
\eta_{i}^{\text {univ }}=\eta^{i}(\Phi) \otimes_{\mathbb{Z}_{p}} \mathbb{Q}_{p}
$$

as in [43, page 664]. Define $u_{0}^{\text {univ }}: \eta_{0}^{\text {univ }} \rightarrow T_{0}^{\text {univ }}$ by $u_{0}^{\text {univ }}\left(e_{0,0}\right)=z t_{0}$ and $u_{0}^{\text {univ }}\left(e_{0,1}\right)=t_{0}$, and $u_{1}^{\text {univ }}: \eta_{1}^{\text {univ }} \rightarrow T_{1}^{\text {univ }}$ by $u_{1}^{\text {univ }}\left(e_{1,0}\right)=(p / z) t_{1}$ and $u_{1}^{\text {univ }}\left(e_{1,1}\right)=t_{1}$, where $z$ denotes the standard coordinate function on $\mathcal{H}_{p}$. Define $\eta^{\text {univ }}=\eta_{0}^{\text {univ }} \oplus \eta_{1}^{\text {univ }}$ and similarly define $u^{\text {univ }}=u_{0}^{\text {univ }} \oplus u_{1}^{\text {univ }}$. We write $\rho^{\text {univ }}:\left(\mathbb{Q}_{p}\right)_{\mathcal{H}_{p}}^{2} \stackrel{\sim}{\rightarrow} \eta_{0}^{\text {univ }}$ for the isomorphism determined by the choice of the basis $\left\{e_{0,0}, e_{0,1}\right\}$. For $\gamma=\left(\begin{array}{ll}a & b \\ c & d\end{array}\right) \in \mathbf{M}_{2}\left(\mathbb{Q}_{p}\right)$ and $i=0,1$, define endomorphisms $\phi_{i}^{(\gamma)}$ in $\operatorname{End}_{\mathcal{O}_{\mathcal{H}_{p}}}\left(T_{i}^{\text {univ }}\right)$ by

$$
\begin{aligned}
\phi_{0}^{(\gamma)}\left(f(z) \otimes t_{0}\right) & =(c z+d) f(\gamma(z)) \otimes t_{0}, \\
\phi_{1}^{(\gamma)}\left(f(z) \otimes t_{1}\right) & =(a+b / z) f(\gamma(z)) \otimes t_{1}
\end{aligned}
$$

for any $f \in \mathcal{O}_{\mathcal{H}_{p}}(U)$, and any affinoid $U \subseteq \mathcal{H}_{p}$. Define an action of $\operatorname{SL}_{2}\left(\mathbb{Q}_{p}\right)$ on $\eta_{i}^{\text {univ }}$ for $i=0,1$ to make $u_{i}^{\text {univ }}$ equivariant with respect to these actions; in other words, for $\gamma=\left(\begin{array}{ll}a & b \\ c & d\end{array}\right)$ in $\mathrm{GL}_{2}\left(\mathbb{Q}_{p}\right)$, put $\gamma^{*}\left(\begin{array}{l}x_{0,0} \\ x_{0,1}\end{array}\right)=\left(\begin{array}{ll}a & b \\ c & d\end{array}\right)\left(\begin{array}{l}x_{0,0} \\ x_{0,1}\end{array}\right)$ and $\gamma^{*}\left(\begin{array}{l}x_{1,0} \\ x_{1,1}\end{array}\right)=\left(\begin{array}{cc}a & b / p \\ p c & d\end{array}\right)\left(\begin{array}{l}x_{1,0} \\ x_{1,1}\end{array}\right)$. Let $\mathbb{Z}_{p}[\Pi]$ act on $T^{\text {univ }}$ by $\Pi t_{0}=(p / z) t_{1}$ and $\Pi t_{1}=z t_{0}$. We let $\mathbb{Z}_{p}[\Pi]$ act on $\eta^{\text {univ }}$ in such a way that $u^{\text {univ }}$ commutes with this action. We call the quadruplet

$$
\mathcal{D}^{\text {univ }}=\left(\eta^{\text {univ }}, T^{\text {univ }}, u^{\text {univ }}, \rho^{\text {univ }}\right)
$$

the universal rigid data.

Passing to the associated normed sheaves [43, Definition 6], we obtain from $\mathcal{D}^{\text {univ }}$ a quadruplet $\hat{\mathcal{D}}^{\text {univ }}=\left(\hat{\eta}^{\text {univ }}, \hat{T}^{\text {univ }}, \hat{u}^{\text {univ }}, \hat{\rho}^{\text {univ }}\right)$ on $\hat{\mathcal{H}}_{p}$, corresponding to a $\hat{\mathcal{H}}_{p}$-valued point, which is universal in the following sense: for each $B \in \operatorname{Nilp}$ and each $\Psi: S=\operatorname{Spec}(B) \rightarrow$ $\hat{\mathcal{H}}_{p}$ corresponding to a quadruplet $(\eta, T, u, \rho)$, we have

$$
(\eta, T, u, \rho)=\left(\Psi^{-1} \hat{\eta}^{\text {univ }}, \Psi^{*} \hat{T}^{\text {univ }}, \Psi^{-1} \hat{u}^{\text {univ }}, \Psi^{-1} \hat{\rho}^{\text {univ }}\right) .
$$

See [43, Corollary 18 and Theorem 19] for more precise and complete statements. We call $\hat{\mathcal{D}}^{\text {univ }}$ the universal formal data, and we denote the quadruplet on the RHS of (20) by $\hat{\mathcal{D}}_{\Psi}^{\text {univ }}$ to simplify the notation. 
The universal SFD-module $\mathcal{G}$ over $\hat{\mathcal{H}}_{p}^{\text {unr }}$ can be recovered from a universal rigid data $\mathcal{D}^{\text {univ }}$. Pulling back via the projection $\pi_{\hat{\mathcal{H}}_{p}}: \hat{\mathcal{H}}_{p}^{\text {unr }} \rightarrow \hat{\mathcal{H}}_{p}$, we obtain a quadruplet

$$
\hat{\mathcal{D}}^{\text {unr }}=\left(\hat{\eta}^{\text {unr }}, \hat{T}^{\text {unr }}, \hat{u}^{\text {unr }}, \hat{\rho}^{\text {unr }}\right)=\left(\pi_{\hat{\mathcal{H}}_{p}}^{-1} \hat{\eta}^{\text {univ }}, \pi_{\hat{\mathcal{H}}_{p}}^{*} \hat{T}^{\text {univ }}, \pi_{\hat{\mathcal{H}}_{p}}^{-1} \hat{u}^{\text {univ }}, \pi_{\hat{\mathcal{H}}_{p}}^{-1} \hat{\rho}^{\text {univ }}\right)
$$

on $\hat{\mathcal{H}}_{p}^{\text {unr }}$. Comparing (20) with the universal property satisfied by $\mathcal{G}$, we see that the quadruplet $\left(\eta_{\mathcal{G}}, T_{\mathcal{G}}, u_{\mathcal{G}}, \rho_{\mathcal{G}}\right)$ associated to $\mathcal{G}$ coincides with the quadruplet $\hat{\mathcal{D}}^{\text {unr }}$. In particular, the associated quadruplet $\left(\eta_{\mathcal{G}}^{\text {rig }}, T_{\mathcal{G}}^{\text {rig }}, u_{\mathcal{G}}^{\text {rig }}, \rho_{\mathcal{G}}^{\text {rig }}\right)$ on the rigid $\hat{\mathbb{Q}}_{p}^{\text {unr }}$-rigid analytic space $\mathcal{G}^{\text {rig }}$ is the quadruplet

$$
\mathcal{D}^{\text {unr }}=\left(\eta^{\text {unr }}, T^{\text {unr }}, u^{\text {unr }}, \rho^{\text {unr }}\right)=\left(\pi_{\mathcal{H}_{p}^{\text {unr }}}^{-1} \eta^{\text {univ }}, \pi_{\mathcal{H}_{p}^{\text {unr }}}^{*} T^{\text {univ }}, \pi_{\mathcal{H}_{p}^{\text {unr }}}^{-1} u^{\text {univ }}, \pi_{\mathcal{H}_{p}^{\text {unr }}}^{-1} \rho^{\text {univ }}\right)
$$

obtained from the quadruplet $\mathcal{D}^{\text {univ }}$, where $\pi_{\mathcal{H}_{p}^{\text {unr }}}: \mathcal{H}_{p}^{\text {unr }} \rightarrow \mathcal{H}_{p}$ is the canonical projection.

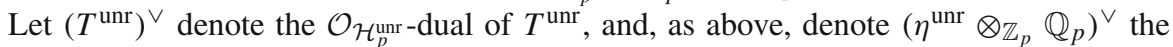
$\mathbb{Q}_{p}$-linear dual of $\eta^{\text {unr }} \otimes_{\mathbb{Z}_{p}} \mathbb{Q}_{p}$. From the surjective map $u^{\text {unr }}: \eta^{\text {unr }} \otimes_{\mathbb{Z}_{p}} \mathcal{O}_{\mathcal{H}_{p}^{\text {unr }}} \rightarrow T^{\text {unr }}$ induced by $u^{\text {univ }}$ we obtain an injective map

$$
\tau:\left(T^{\mathrm{unr}}\right)^{\vee} \longleftrightarrow\left(\eta^{\mathrm{unr}} \otimes_{\mathbb{Z}_{p}} \mathbb{Q}_{p}\right)^{\vee} \otimes_{\mathbb{Q}_{p}} \mathcal{O}_{\mathcal{H}_{p}^{\text {unr }}}
$$

Proposition 4.2 We have canonical isomorphisms

$$
\begin{aligned}
& \left(T^{\text {unr }}\right)^{\vee} \stackrel{\sim}{\longrightarrow} \omega_{\mathcal{G}}^{\text {rig }}, \\
& \left(\eta^{\text {unr }} \otimes_{\mathbb{Z}_{p}} \mathbb{Q}_{p}\right)^{\vee} \otimes_{\mathbb{Q}_{p}} \mathcal{O}_{\mathcal{H}_{p}^{\text {unr }}} \stackrel{\sim}{\longrightarrow} \mathcal{H}_{\mathrm{dR}}^{1, \text { rig }}(\mathcal{G}) .
\end{aligned}
$$

under which the map $\tau$ corresponds to the first map in (15).

Proof The first statement follows from the canonical isomorphism between $T_{\mathcal{G}}=\mathcal{L i e}_{\mathcal{G}}$ and $T^{\mathrm{unr}}$, while the second follows from Proposition 4.1 combined with (16). For the statement about $\tau$, note that for each SFD-module $G$ over $\overline{\mathbb{F}}_{p}$, the map $u_{G}$ corresponds under the identification between $\eta(G) \otimes_{\mathbb{Z}_{p}} \hat{\mathbb{Q}}_{p}^{\text {unr }}$ and $\mathbb{M}(G) \otimes_{\hat{\mathbb{Z}}_{p}^{\text {unr }}} \hat{\mathbb{Q}}_{p}^{\text {unr }}$ to the canonical projection $\mathbb{M}(G) / \mathbb{V}_{G} \mathbb{M}(G) \rightarrow T_{G}$, where $T_{G}$ is the tangent space of $G$ at the origin.

\subsection{The action of the idempotent $e$}

Fix an isomorphism $\mathbb{Q}_{p}\left(\sqrt{p_{0}}\right) \simeq \mathbb{Q}_{p^{2}}$. By means of this isomorphism, and the fixed embed$\operatorname{ding} \mathbb{Q}_{p^{2}} \hookrightarrow D$, we may identify elements $a+b \sqrt{p_{0}}$ in $\mathbb{Q}_{p}\left(\sqrt{p_{0}}\right)$ (where $a, b \in \mathbb{Q}_{p}$ ) with elements of $D$ in what follows without explicitly mentioning it.

Lemma $4.3 e \cdot\left(\eta(\Phi) \otimes_{\mathbb{Z}_{p}} \mathbb{Z}_{p^{2}}\right)=\eta^{0}(\Phi) \otimes_{\mathbb{Z}_{p}} \mathbb{Z}_{p^{2}}$ and $e \cdot\left(T(\Phi) \otimes_{\mathbb{Z}_{p}} \mathbb{Z}_{p^{2}}\right)=T^{0}(\Phi) \otimes_{\mathbb{Z}_{p}}$ $\mathbb{Z}_{p^{2}}$.

Proof The action of $\mathcal{O}_{D}$ on $\eta(\Phi)$ is induced by duality from the action on $\mathbb{M}(\Phi)$, so any element $a \in \mathbb{Z}_{p^{2}} \hookrightarrow \mathcal{O}_{D}$ acts on $\eta^{0}(\Phi)$ by multiplication by $a$ and on $\eta^{1}(\Phi)$ by multiplication by $\bar{a}$. On the other hand, the action of $1 \otimes a$ on $\eta(\Phi) \otimes \mathbb{Q}_{p} \mathbb{Q}_{p^{2}}$ is given by multiplication by $a$. An immediate calculation shows then that the action of $e$ is just the projection $\eta(\Phi) \rightarrow \eta^{0}(\Phi)$. The argument for $T(\Phi)$ is similar.

$$
\text { Write } \eta_{0}^{\text {unr }}=\pi_{\mathcal{H}_{p}^{\text {unr }}}^{-1} \eta_{0}^{\text {univ }}, T_{0}^{\text {unr }}=\pi_{\mathcal{H}_{p}^{\text {unr }}}^{*} T_{0}^{\text {univ }}, u_{0}^{\text {unr }}=\pi_{\mathcal{H}_{p}^{\text {unr }}}^{-1} u_{0}^{\text {univ }}
$$


Proposition $4.4 e \cdot \eta^{\mathrm{unr}}=\eta_{0}^{\mathrm{unr}}$ and $e \cdot T^{\mathrm{unr}}=T_{0}^{\mathrm{unr}}$.

Proof This is clear from Lemma 4.3 and (18).

For $i=0,1$, the sheaf $T_{0}^{\mathrm{unr}}$ is a free $\mathcal{O}_{\mathcal{H}_{p}^{\text {unr }}}$-module of rank 1 , so it is invertible; denote

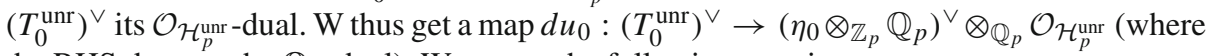
the RHS denotes the $\mathbb{Q}_{p}$-dual). We set up the following notation:

- $\underline{\omega}_{\mathcal{G}}^{0}=e \cdot \underline{\omega}_{\mathcal{G}}^{\text {rig }}$;

- $\mathcal{L}_{\mathcal{G}}^{0}=e \cdot \mathcal{H}_{\mathrm{dR}}^{1, \text { rig }}(\mathcal{G})$.

Applying the idempotent $e$ and using Propositions 4.2 and 4.4 we then obtain a diagram with exact rows in which the vertical arrows are isomorphisms:

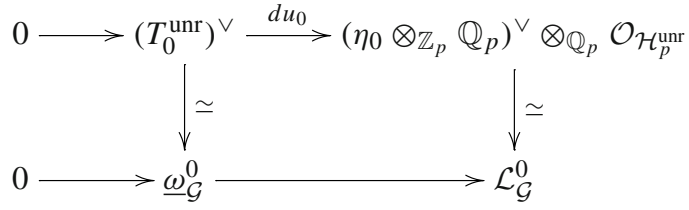

\subsection{Differential calculus on the $p$-adic upper half plane}

We now set up the following notation. Recall that the map $u_{0}$ takes $x_{0,0} e_{0,0}+x_{0,1} e_{0,1}$ to $\left(z x_{0,0}+x_{0,1}\right) \otimes t_{0}$; dualizing, $d u_{0}$ can be described in coordinates by the map which takes the canonical generator $t_{0}$ of the $\mathcal{O}_{\mathcal{H}_{p}^{\text {unr }}}$-module $\left(T_{0}^{\text {unr }}\right)^{\vee}$ (satisfying the relation $d t_{0}\left(t_{0}\right)=1$ ) to the map $x_{0,0} e_{0,0}+x_{0,1} e_{0,1} \mapsto z x_{0,0}+x_{0,1}$. If we denote $d e_{0, i}$ the dual basis of $e_{0, i}$ (satisfying the condition $\left.d e_{0, i}\left(e_{0, j}\right)=\delta_{i, j}\right)$, we may write this map as $z d e_{0,0}+d e_{0,1}$. To simplify the notation, we put from now on $\tau=t_{0}, d \tau=d t_{0}, x=e_{0,0}, y=e_{0,1}, d x=d e_{0,0}$ and $d y=d e_{0,1}$, so that the above map reads simply as

$$
d \tau=z d x+d y .
$$

Let $\mathcal{C}^{0}=\mathcal{C}^{0}\left(\mathcal{H}_{p}\left(\hat{\mathbb{Q}}_{p}^{\text {unr }}\right), \mathbb{C}_{p}\right)$ denote the $\mathbb{C}_{p}$-vector space of continuous (for the standard $p$-adic topology on both spaces) $\mathbb{C}_{p}$-valued functions on $\mathcal{H}_{p}\left(\hat{\mathbb{Q}}_{p}^{\text {unr }}\right)$. Denote $\mathcal{A}=$ $H^{0}\left(\mathcal{H}_{p}^{\text {unr }}, \mathcal{O}_{\mathcal{H}_{p}^{\text {unr }}}\right)$ the $\mathbb{Q}_{p}^{\text {unr }}$-vector space of global sections of $\mathcal{O}_{\mathcal{H}_{p}^{\text {unr }}}$. Each $f \in \mathcal{A}$ is, in particular, continuous on $\mathcal{H}_{p}^{\text {unr }}$ for the standard $p$-adic topology of $\mathbb{Q}_{p}^{\text {unr }}-\mathbb{Q}_{p}$, and therefore restriction induces a map of $\mathbb{Q}_{p}^{\text {unr }}$-vector spaces $r: \mathcal{A} \rightarrow \mathcal{C}^{0}$. Denote $\mathcal{A}^{*}$ the image of the morphism of $\mathcal{A}$-algebras $\mathcal{A}[X, Y] \rightarrow \mathcal{C}$ defined by sending $X$ to the function $z \mapsto 1 /(z-\sigma(z))$ and $Y$ to the function $z \mapsto \sigma(z)$ (note that the function $z \mapsto z-\sigma(z)$ is invertible on $\left.\mathcal{H}_{p}^{\text {unr }}\left(\hat{\mathbb{Q}}_{p}^{\text {unr }}\right)\right)$. To simplify the notation, we put from now on

$$
z^{*}=\sigma(z) .
$$

Set up the following notation (here $n \geq 1$ is an integer)

- $\Lambda_{\mathcal{G}}=H^{0}\left(\mathcal{H}_{p}^{\mathrm{unr}}, \mathcal{L}_{\mathcal{G}}^{0}\right)$ and $\Lambda_{\mathcal{G}, n}=\Lambda_{\mathcal{G}}^{\otimes n}$,

- $\Lambda_{\mathcal{G}}^{*}=\Lambda_{\mathcal{G}} \otimes_{\mathcal{A}} \mathcal{A}^{*}$ and $\Lambda_{\mathcal{G}, n}^{*}=\left(\Lambda_{\mathcal{G}, n}^{*}\right)^{\otimes n}$,

- $w_{\mathcal{G}}=H^{0}\left(\mathcal{H}_{p}^{\mathrm{unr}}, \underline{\omega}_{\mathcal{G}}^{0}\right)$ and $w_{\mathcal{G}, n}=w_{\mathcal{G}}^{\otimes n}$,

- $w_{\mathcal{G}}^{*}=w_{\mathcal{G}} \otimes_{\mathcal{A}} \mathcal{A}^{*}$ and $w_{\mathcal{G}, n}^{*}=\left(w_{\mathcal{G}, n}^{*}\right)^{\otimes n}$. 
The $\hat{\mathbb{Q}}_{p}^{\text {unr }}$-algebra $\mathcal{A}$ is equipped with the standard derivation $\frac{d}{d z}$ on power series. The $\mathcal{A}$-module $\Omega_{\mathcal{A}}^{1}=H^{0}\left(\mathcal{H}_{p}^{\text {unr }}, \Omega_{\mathcal{H}_{p}^{\text {unr }}}^{1}\right)$ is then one dimensional and generated by $d z$ satisfying $d z\left(\frac{d}{d z}\right)=1$. We extend the differential operator $\frac{d}{d z}$ to a differential operator $\frac{\partial}{\partial z}: \mathcal{A}^{*} \rightarrow \mathcal{A}^{*}$ by $\hat{\mathbb{Q}}_{p}^{\text {unr }}$-linearity using the product formula and setting $\frac{\partial}{\partial z}\left(z^{*}\right)=0$ and $\frac{\partial}{\partial z}\left(\frac{1}{z-z^{*}}\right)=\frac{-1}{\left(z-z^{*}\right)^{2}}$. Similarly, we define a differential operator $\frac{\partial}{\partial z^{*}}: \mathcal{A}^{*} \rightarrow \mathcal{A}^{*}$ setting $\frac{\partial}{\partial z^{*}}(z)=0, \frac{\partial}{\partial z^{*}}\left(z^{*}\right)=1$ and $\frac{\partial}{\partial z^{*}}\left(\frac{1}{z-z^{*}}\right)=\frac{1}{\left(z-z^{*}\right)^{2}}$. Define $\Omega_{\mathcal{A}^{*}}^{1}$ to be the $\mathcal{A}^{*}$-subalgebra of the algebra of derivations generated by $d z$ and $d z^{*}$ satisfying the usual rules $d z\left(\frac{\partial}{\partial z}\right)=1, d z\left(\frac{\partial}{\partial z^{*}}\right)=0, d z^{*}\left(\frac{\partial}{\partial z^{*}}\right)=0$, $d z^{*}\left(\frac{\partial}{\partial z^{*}}\right)=1$.

\subsection{Splitting of the rigid analytic Hodge filtration}

Recall the notation fixed before for the differential form $d \tau=z d x+d y$. Define

$$
d \tau^{*}=z^{*} d x+d y .
$$

Then $d \tau^{*}$ belongs to $w_{\mathcal{G}}^{*}$. Taking global sections, restricting to $\hat{\mathbb{Q}}_{p}^{\text {unr }}$, and extending linearly with $\mathcal{A}^{*}$ we obtain a short exact sequence of $\mathcal{A}^{*}$-algebras

$$
0 \longrightarrow w_{\mathcal{G}}^{*} \longrightarrow \Lambda_{\mathcal{G}}^{*}
$$

Theorem 4.5 The exact sequence (22) admits a canonical splitting $\Psi_{p}: \Lambda_{\mathcal{G}}^{*} \rightarrow w_{\mathcal{G}}^{*}$.

Proof We have

$$
d x=\frac{d \tau-d \tau^{*}}{z-z^{*}}, \quad d y=\frac{z d \tau^{*}-z^{*} d \tau}{z-z^{*}} .
$$

We may therefore write any differential form $\omega=f(z) d x+g(z) d y$ with $f, g \in \mathcal{A}^{*}$ as

$$
\omega=\left(\frac{f(z)-g(z) z^{*}}{z-z^{*}}\right) d \tau+d \tau^{*}\left(\frac{z g(z)-f(z)}{z-z^{*}}\right) .
$$

One then defines the sough-for splitting sending $\omega \mapsto\left(\frac{f(z)-g(z) z^{*}}{z-z^{*}}\right) d \tau$.

\subsection{The $p$-adic Maass-Shimura operator}

Taking global section, the Gauss-Manin connection gives rise to a map $\nabla_{\mathcal{G}}^{\text {rig }}: \Lambda_{\mathcal{G}} \rightarrow$ $\Lambda_{\mathcal{G}} \otimes \Omega_{\mathcal{A}}^{1}$. We extend $\nabla_{\mathcal{G}}^{\text {rig }}$ to a map $\nabla_{\mathcal{G}}^{*}: \Lambda_{\mathcal{G}}^{*} \rightarrow \Lambda_{\mathcal{G}}^{*} \otimes \Omega_{\mathcal{A}^{*}}^{1}$ as follows. First define $\nabla_{\mathcal{G}}^{1,0}: \Lambda_{\mathcal{G}}^{*} \rightarrow \Lambda_{\mathcal{G}}^{*} \otimes \Omega_{\mathcal{A}^{*}}^{1}$ to be the derivation satisfying the rules $\nabla_{\mathcal{G}}^{1,0}(d \tau)=d x \otimes d z$, $\nabla_{\mathcal{G}}^{1,0}\left(d \tau^{*}\right)=0, \nabla_{\mathcal{G}}^{1,0}(z)=1, \nabla_{\mathcal{G}}^{1,0}\left(z^{*}\right)=0$. Define similarly the derivation $\nabla_{\mathcal{G}}^{0,1}: \Lambda_{\mathcal{G}}^{*} \rightarrow$ $\Lambda_{\mathcal{G}}^{*} \otimes \Omega_{\mathcal{A}^{*}}^{1}$ by the rules $\nabla_{\mathcal{G}}^{0,1}(d \tau)=0, \nabla_{\mathcal{G}}^{0,1}\left(d \tau^{*}\right)=d x \otimes d z^{*}, \nabla_{\mathcal{G}}^{0,1}(z)=0, \nabla_{\mathcal{G}}^{0,1}(\bar{z})=d z^{*}$. We finally define

$$
\nabla_{\mathcal{G}}^{*}=\nabla_{\mathcal{G}}^{1,0}+\nabla_{\mathcal{G}}^{0,1}: \Lambda_{\mathcal{G}}^{*} \longrightarrow \Lambda_{\mathcal{G}}^{*} \otimes \Omega_{\mathcal{A}^{*}}^{1}
$$

Taking global sections, the Kodaira-Spencer map gives rise to a map $\mathrm{KS}_{\mathcal{G}}: w_{\mathcal{G}}^{\otimes 2} \rightarrow \Omega_{\mathcal{A}}^{1}$, which we extend $\mathcal{A}^{*}$-linearly to a map

$$
\mathrm{KS}_{\mathcal{G}}^{*}:\left(w_{\mathcal{G}}^{*}\right)^{\otimes 2} \longrightarrow \Omega_{\mathcal{A}^{*}}^{1}
$$


Note that

$$
\nabla_{\mathcal{G}}^{\mathrm{rig}}(d \tau)=\nabla_{\mathcal{G}}^{*}(d \tau)=\frac{d \tau-d \tau^{*}}{z-z^{*}} \otimes d z
$$

and, since $\nabla_{\mathcal{G}}^{*}\left(z^{*}\right)=0$, we have

$$
\nabla_{\mathcal{G}}^{*}\left(d \tau^{*}\right)=0 .
$$

In particular, if $f(z) \otimes d \tau \in w_{\mathcal{G}}^{*}$ we have

$$
\nabla_{\mathcal{G}}^{\mathrm{rig}}(f(z) \otimes d \tau)=\left(\frac{\partial}{\partial z} f(z) \otimes d \tau+f(z) \otimes \frac{d \tau-d \tau^{*}}{z-z^{*}}\right) \otimes d z .
$$

Taking global sections, we can form the pairing $\langle,\rangle_{\mathcal{G}}^{\text {rig }}: \Lambda_{\mathcal{G}} \otimes_{\mathcal{A}} \Lambda_{\mathcal{G}} \rightarrow \mathcal{A}$. Extending linearly by $\mathcal{A}^{*}$, we obtain a new pairing

$$
\langle,\rangle_{\mathcal{G}}^{*}: \Lambda_{\mathcal{G}}^{*} \otimes_{\mathcal{A}^{*}} \Lambda_{\mathcal{G}}^{*} \longrightarrow \mathcal{A}^{*} .
$$

Using the description of the Kodaira-Spencer map in Sect. 4.4, we see that

$$
\left\langle d \tau, \nabla_{\mathcal{G}}^{\mathrm{rig}}(d \tau)\right\rangle_{\mathcal{G}}^{\mathrm{rig}}=\left\langle d \tau, \nabla_{\mathcal{G}}^{*}(d \tau)\right\rangle_{\mathcal{G}}^{*}=\frac{-\left\langle d \tau, d \tau^{*}\right\rangle_{\mathcal{G}}^{*}}{z-z^{*}} d z=-\langle d x, d y\rangle_{\mathcal{G}}^{*} d z
$$

where for the second equality we use (23), while the last equality follows easily from the equation

$$
\left\langle z d x+d y, z^{*} d x+d y\right\rangle_{\mathcal{G}}^{*}=\left(z-z^{*}\right)\langle d x, d y\rangle_{\mathcal{G}}^{*} .
$$

Therefore

$$
\mathrm{KS}_{\mathcal{G}}^{\mathrm{rig}}(d \tau \otimes d \tau)=-\langle d x, d y\rangle_{\mathcal{G}}^{\mathrm{rig}} d z
$$

So, to compute $\mathrm{KS}_{\mathcal{G}}^{*}(d \tau \otimes d \tau)=\mathrm{KS}_{\mathcal{G}}^{\text {rig }}(d \tau \otimes d \tau)$ we are reduced to compute $\langle d x, d y\rangle_{\mathcal{G}}^{\text {rig }}$. For this, we switch to de Rham homology and follow the computations in $[19,37]$.

To begin with, let $W$ denote the order $\mathcal{O}_{D}$ viewed as free left $\mathcal{O}_{D}$-module of rank 1 ; then $W \simeq \mathcal{R}_{\max } \otimes_{\mathbb{Z}} \mathbb{Z}_{p}$. By [3, Ch. III, Lemma 1.9], the collection of bilinear skew-symmetric maps $\psi: W \times W \rightarrow \mathbb{Z}_{p}$ which satisfy $\psi(d x, y)=\psi\left(x, d^{\dagger} y\right)$ (for all $x, y \in W$ and $d \in \mathcal{O}_{D}$ ) is a free $\mathbb{Z}_{p}$-module of rank 1 , and every generator $\psi_{0}$ of this $\mathbb{Z}_{p}$-module is a perfect duality on $W$; the pairing

$$
\psi_{0}(x, y)=\frac{\operatorname{tr}\left(\mathbf{i} y^{\dagger} x\right)}{p}
$$

is such a generator, which we fix once and for all (recall the notation introduced in Sects. 2.1 and 2.3 for $\mathbf{i}$ and $d^{\dagger}$ ).

Recall that $H_{\text {cris }}^{1}(\Phi)$ is a free $D \otimes \mathbb{Q}_{p} \hat{\mathbb{Q}}_{p}^{\text {unr }}$-module of rank 1 ( $c f$. [23, page 354]); the structure of $D \otimes \mathbb{Q}_{p} \hat{\mathbb{Q}}_{p}^{\text {unr }}$-module is induced from the $D$-module structure of $\left(\eta(\Phi) \otimes_{\mathbb{Z}_{p}} \mathbb{Q}_{p}\right)^{\vee}$ via the isomorphisms $\left(\eta(\Phi) \otimes_{\mathbb{Z}_{p}} \mathbb{Q}_{p}\right)^{\vee} \simeq V_{\text {cris }}(\Phi)$ and $H_{\text {cris }}^{1}(\Phi) \simeq V_{\text {cris }}(\Phi) \otimes_{\mathbb{Q}_{p}} \hat{\mathbb{Q}}_{p}^{\text {unr }}$ in Lemma 4.1. We have then from Lemma 4.1 then canonical isomorphisms of convergent $F$-isocrystals:

$$
\begin{aligned}
\mathcal{H}_{\mathrm{dR}}^{1}\left(\mathcal{G} / \hat{\mathcal{H}}_{p}\right) & \simeq H_{\mathrm{cris}}^{1}(\Phi) \otimes_{\hat{\mathbb{Q}}_{p}^{\mathrm{unr}}} \mathcal{E}\left(\mathcal{O}_{\hat{\mathcal{H}}_{p}^{\mathrm{unr}}}\right) \\
& \simeq\left(D \otimes_{\mathbb{Q}_{p}} \hat{\mathbb{Q}}_{p}^{\mathrm{unr}}\right) \otimes_{\hat{\mathbb{Q}}_{p}^{\text {unr }}} \mathcal{E}\left(\mathcal{O}_{\hat{\mathcal{H}}_{p}^{\text {unr }}}\right) \\
& \simeq\left(D \otimes_{\mathbb{Q}_{p}} \mathbb{Q}_{p^{2}}\right) \otimes_{\mathbb{Q}_{p^{2}}} \mathcal{E}\left(\mathcal{O}_{\hat{\mathcal{H}}_{p}^{\text {unr }}}\right) \\
& \simeq \mathrm{M}_{2}\left(\mathbb{Q}_{p^{2}}\right) \otimes_{\mathbb{Q}_{p} 2} \mathcal{E}\left(\mathcal{O}_{\hat{\mathcal{H}}_{p}^{\text {unr }}}\right) .
\end{aligned}
$$


Under the isomorphism (26), the $\hat{\mathbb{Q}}_{p}^{\text {unr }}$-linear extension of $\psi_{0}$ defines a pairing

$$
\psi_{0}: H_{\text {cris }}^{1}(\Phi) \times H_{\text {cris }}^{1}(\Phi) \longrightarrow \hat{\mathbb{Q}}_{p}^{\text {unr }}
$$

still denoted with the same symbol. If we denote $\langle,\rangle_{\mathcal{G}}^{\text {rig }}$ the restriction of $\langle,\rangle_{\mathcal{G}}^{\text {rig }}$ to $H_{\text {cris }}^{1}(\Phi)$, it follows from the unicity of $\psi_{0}$ up to constant that there exists an element $t_{p} \in \mathbb{C}_{p}^{\times}$such that

$$
\langle,\rangle_{\mathcal{G}, W}^{\text {rig }}=\frac{1}{t_{p}} \cdot \psi_{0} .
$$

Moreover, under the isomorphism (26), the element $d \tau=z d x+d y$ of $\mathcal{H}_{\mathrm{dR}}^{1}(\mathcal{G})$ corresponds to the element $e_{1} \otimes z+e_{2} \otimes 1$, where $e_{1}=\left(\begin{array}{ll}1 & 0 \\ 0 & 0\end{array}\right), e_{2}=\left(\begin{array}{ll}0 & 1 \\ 0 & 0\end{array}\right), e_{3}=\left(\begin{array}{ll}0 & 0 \\ 1 & 0\end{array}\right), e_{4}=\left(\begin{array}{ll}0 & 0 \\ 0 & 1\end{array}\right)$ is the standard basis of $\mathrm{M}_{2}\left(\mathbb{Q}_{p^{2}}\right)$. We therefore obtain the sought-for recipe to compute the Kodaira-Spencer image of $d \tau \otimes d \tau$ in terms of $\psi_{0}$ :

$$
\mathrm{KS}_{\mathcal{G}}^{\mathrm{rig}}(d \tau \otimes d \tau)=\frac{1}{t_{p}} \cdot \psi_{0}\left(e_{1} \otimes z, e_{2} \otimes 1\right) .
$$

Remark 4.6 The number $t_{p}$ may be viewed as the $p$-adic analogue of the complex period $2 \pi i$, relating de Rham cohomology with homology [37, (2.7)], [19, p. 4197]. This explains why we prefer to keep $t_{p}$ at the denominator in (27).

We now make more explicit the equations (23) and (28) using Hashimoto basis $\{\mathbf{1}, \mathbf{i}, \mathbf{j}, \mathbf{k}\}$ fixed in Sect. 2.1. As in [37, Proposition 2.3] and [18, (2)], define $\epsilon_{1}=\mathbf{1}, \epsilon_{2}=(\mathbf{1}+\mathbf{j}) / 2$, $\epsilon_{3}=(\mathbf{i}+\mathbf{i j}) / 2, \epsilon_{4}=\left(a p N^{-} \mathbf{j}+\mathbf{i j}\right) / p_{0}$ and use these elements to define a symplectic basis of $W$ with respect to the pairing $\psi_{0}$ as in $[18,(5)]$ by $\eta_{1}=\epsilon_{3}-\frac{p_{0}-1}{2} \epsilon_{4}, \eta_{2}=-a D \epsilon_{1}-\epsilon_{4}$, $\eta_{3}=\epsilon_{1}, \eta_{4}=\epsilon_{2}$ (note that $\psi_{0}$ we consider above is equal to the pairing $(x, y) \mapsto \operatorname{tr}\left(x i y^{\dagger}\right)$ in [18, (3)]). Denote $\eta_{1}^{\vee}, \eta_{2}^{\vee}, \eta_{3}^{\vee}, \eta_{4}^{\vee}$ the dual basis of $W^{\vee}$, and let $\eta^{\vee}$ be the column vector with entries $\eta_{1}^{\vee}, \eta_{2}^{\vee}, \eta_{3}^{\vee}, \eta_{4}^{\vee}$. The elements $\eta_{i}^{\vee}$ give rise to elements of $\mathcal{H}_{\mathrm{dR}}^{1}(\mathcal{G})$, denoted with the same symbol, which are horizontal with respect to $\nabla_{\mathcal{G}}^{\text {rig }}$, namely $\nabla_{\mathcal{G}}^{\text {rig }}\left(\eta_{i}^{\vee}\right)=0$. Write $d \tau=\Pi(z) \cdot \underline{\eta}^{\vee}$. A simple calculation shows that

$$
\Pi(z)=\left(\frac{\alpha^{-}}{2 \sqrt{p_{0}}}\left(\alpha^{+} a \Delta z+1\right), \frac{-1}{\sqrt{p_{0}}}\left(\alpha^{+} a \Delta z+1\right), z, \frac{1}{2} \alpha^{+} z\right) .
$$

Since $\eta_{i}^{\vee}$ are horizontal sections of $\nabla_{\mathcal{G}}^{\text {rig }}$, using (29) to calculate $\frac{d \Pi(z)}{d z}$ shows that (23) becomes

$$
\nabla_{\mathcal{G}}^{\mathrm{rig}}(d \tau)=\left(\frac{\alpha^{-} \alpha^{+} a \Delta}{2 \sqrt{p_{0}}}, \frac{-\alpha^{+} a \Delta z}{\sqrt{p_{0}}}, 1, \frac{1}{2} \alpha^{+}\right) \cdot \underline{\eta}^{\vee} \otimes d z .
$$

The recipe (17) to compute the Kodaira-Spencer map combined with (27) and (30) gives then

$$
\mathrm{KS}_{\mathcal{G}}^{\mathrm{rig}}(d \tau \otimes d \tau)=\frac{1}{t_{p}} \frac{d \Pi(z)}{d z}\left(\begin{array}{cc}
0 & I_{2} \\
-I_{2} & 0
\end{array}\right) \Pi(z)^{T} d z=\frac{1}{t_{p}} d z .
$$

In particular, (31) shows that $\mathrm{KS}_{\mathcal{G}}^{\text {rig }}$ is an isomorphism, and therefore the same is true for $\mathrm{KS}_{\mathcal{G}}^{*}$. This allows us to define the operator

$$
\tilde{\nabla}_{\mathcal{G}, n}^{*}: \Lambda_{\mathcal{G}, n}^{*} \stackrel{\nabla_{\mathcal{G}, n}^{*}}{\longrightarrow} \Lambda_{\mathcal{G}, n}^{*} \otimes_{\mathcal{A}^{*}} \Omega_{\mathcal{A}^{*}}^{1} \stackrel{\left(\mathrm{KS}_{\mathcal{G}}^{*}\right)^{-1}}{\longrightarrow} \Lambda_{\mathcal{G}, n}^{*} \otimes_{\mathcal{A}^{*}} w_{\mathcal{G}, 2}^{*} \longrightarrow \Lambda_{\mathcal{G}, n}^{*} \otimes_{\mathcal{A}^{*}} \Lambda_{\mathcal{G}, 2}^{*} \longrightarrow \Lambda_{\mathcal{G}, n+2}^{*}
$$

where $\nabla_{\mathcal{G}, n}^{*}$ is obtained from $\nabla_{\mathcal{G}}^{*}$ using the Leibniz rule (see [19, Sect. 3.2]). The splitting in Theorem 4.5 induces a morphism of $\mathcal{A}^{*}$-modules $\Psi_{p, n}: \Lambda_{\mathcal{G}, n}^{*} \rightarrow w_{\mathcal{G}, n}^{*}$. 
Definition 4.7 The composition

$$
\Theta_{p, n}: w_{\mathcal{G}, n}^{*} \longrightarrow \Lambda_{\mathcal{G}, n}^{*} \stackrel{\tilde{\nabla}_{\mathcal{G}, n}^{*}}{\longrightarrow} \Lambda_{\mathcal{G}, n+2}^{*} \stackrel{\Psi_{p, n+2}}{\longrightarrow} w_{\mathcal{G}, n+2}^{*}
$$

is the p-adic Maass-Shimura operator.

We also need to consider iterates of this operator. Define

$$
\tilde{\nabla}_{n}^{j, *}=\tilde{\nabla}_{n+2(j-1)}^{*} \circ \cdots \circ \tilde{\nabla}_{n+2}^{*} \circ \tilde{\nabla}_{n}^{*} .
$$

Define then

$$
\Theta_{p, n}^{j}: w_{\mathcal{G}, n}^{*} \longrightarrow \Lambda_{\mathcal{G}, n}^{*} \stackrel{\tilde{\nabla}_{n}^{j, *}}{\longrightarrow} \Lambda_{\mathcal{G}, n+2 j}^{*} \stackrel{\Psi_{p, n+2 j}}{\longrightarrow} w_{\mathcal{G}, n+2 j}^{*}
$$

where the morphism of $\mathcal{A}^{*}$-modules $\Psi_{p, n+2 j}: \Lambda_{\mathcal{G}, n+2 j}^{*} \rightarrow w_{\mathcal{G}, n+2 j}^{*}$ is induced as above by the splitting in Theorem 4.5. We call $\Theta_{p, n}^{j}$ the $j$-th iterate of the $p$-adic Maass-Shimura operator.

\subsection{Relation with Franc Maass-Shimura operator}

The work accomplished so far allows us to explicitly describe $\Theta_{p, k}^{j}$, thus relating it to Franc Shimura-Masss operator. We first introduce some differential operators, similar in shape to the Maass-Shimura operator in the real analytic setting. For each integer $k \geq 0$, we may then define the $\hat{\mathbb{Q}}_{p}^{\text {unr }}$-linear function

$$
\delta_{p, k}=\frac{\partial}{\partial z}+\frac{k}{z-z^{*}}: \mathcal{A}^{*} \longrightarrow \mathcal{A}^{*}
$$

For each integer $j \geq 0$ we get a map $\delta_{p, k}^{j}: \mathcal{A}^{*} \rightarrow \mathcal{A}^{*}$ defined by

$$
\delta_{p, k}^{j}=\delta_{p, k+2(j-1)} \circ \cdots \circ \delta_{p, k+2} \circ \delta_{p, k} .
$$

We call $\delta_{p, k}$ the Maass-Shimura operator, and $\delta_{p, k}^{j}$ its $j$-th iteration. Applying (31) to compute the inverse of the Kodaira-Spencer map to (25), we obtain

$$
\tilde{\nabla}_{k}^{*}\left(f(z) \otimes d \tau^{\otimes k}\right)=\frac{1}{t_{p}} \cdot\left(\frac{\partial}{\partial z} f(z) \otimes d \tau^{\otimes k+2}+k f(z) \otimes \frac{d \tau-d \sigma(\tau)}{z-\sigma(z)} \otimes d \tau^{\otimes k+1}\right) .
$$

Applying the splitting $\Psi_{p, k+2}$ of the Hodge filtration which annihilate $d \tau^{*}$, we finally obtain

$\Theta_{p, k}\left(f(z) \otimes d \tau^{\otimes k}\right)=\frac{1}{t_{p}} \cdot\left(\frac{\partial}{\partial z} f(z)+\frac{k f(z)}{z-\sigma(z)}\right) \otimes d \tau^{\otimes k+2}=\frac{1}{t_{p}} \cdot \delta_{p, k}(f) \otimes d \tau^{\otimes k+2}$.

Iterating (34) we obtain

$$
\Theta_{p, k}^{j}\left(f(z) \otimes d \tau^{\otimes k}\right)=\left(\frac{1}{t_{p}}\right)^{j} \delta_{p, k}^{j}(f(z)) \otimes d \tau^{\otimes k+2 j} .
$$

This proves Corollary 1.2 stated in the Introduction. 


\section{The $p$-adic Maass-Shimura operator on Shimura curves}

The aim of this section is to apply the results in Sect. 4 to define a $p$-adic Maass-Shimura operator on Shimura curves.

\section{1 -adic uniformization of Shimura curves}

In this subsection we review the Cerednik-Drinfel'd Theorem. Recall the subgroup $V_{1}\left(N^{+}\right)$ of the Eichler order $\mathcal{R}$ of level $N^{+}$of $\mathcal{B}$ defined in Sect. 2.1. Let $U$ be the open compact subgroup of the group $\hat{\mathcal{R}}^{\times}$(where $\hat{\mathcal{R}}$ is the profinite completion of $\mathcal{R}$ ) consisting of elements $\left(x_{\ell}\right)_{\ell} \in \hat{\mathcal{R}}^{\times}$such that $\iota_{\ell}\left(x_{\ell}\right)=\left(\begin{array}{ll}a & b \\ 0 & 1\end{array}\right)$ for elements $a \in \mathbb{Z}_{\ell}^{\times}$and $b \in \mathbb{Z}_{\ell}$, for each prime number $\ell \mid N^{+}$. Let $U^{(p)}$ denote the subgroup of $\hat{\mathcal{R}}^{\times}$consisting of elements whose $\ell$-component belongs to the $\ell$-component of $U$, for all primes $\ell \neq p$. Let $B / \mathbb{Q}$ be the quaternion algebra obtained from $\mathcal{B}$ by interchanging the invariants at $\infty$ and $p$; so $B$ is the definite quaternion algebra over $\mathbb{Q}$ of discriminant $N^{-}$. Fix isomorphisms $\mathcal{B}_{\ell} \simeq B_{\ell}$ for all primes $\ell \neq p$; so $U^{(p)}$ defines a subgroup of $\left(\hat{B}^{\times}\right)^{(p)}$, still denoted with the same symbol $U^{(p)}$ (as above, $\hat{B}$ is the adele ring of $B$ and $\left(\hat{B}^{\times}\right)^{(p)}$ is the subgroup of invertible elements of $\hat{B}$ whose $\ell$-component belongs to the $\ell$-component of $U$ via the isomorphism $\mathcal{B}_{\ell} \simeq B_{\ell}$, for all primes $\ell \neq p$ ). Define $\tilde{\Gamma}_{p}=B^{\times} \cap U^{(p)}$. We still denote $\tilde{\Gamma}_{p}$ the image of $\tilde{\Gamma}_{p}$ in $\mathrm{GL}_{2}\left(\mathbb{Q}_{p}\right)$ via a fixed isomorphism $i_{p}: B \otimes \mathbb{Q}_{\mathbb{Q}} \simeq \mathrm{M}_{2}\left(\mathbb{Q}_{p}\right)$, and we let $\Gamma_{p}$ denote the subgroup of $\tilde{\Gamma}_{p}$ consisting of elements whose determinant has even $p$-power order.

Base changing from $\mathbb{Z}_{p}$ to the valuation ring $\mathbb{Z}_{p^{2}}$ of $\mathbb{Q}_{p^{2}}$ gives a $\mathbb{Z}_{p^{2}}$-formal scheme $\hat{\mathcal{H}}_{p^{2}}$, whose generic fiber $\mathcal{H}_{p^{2}}$ is the base change of the $\mathbb{Q}_{p}$-rigid analytic space $\mathcal{H}_{p}$ to $\mathbb{Q}_{p^{2}}$. The group $\mathrm{GL}_{2}\left(\mathbb{Q}_{p}\right)$ acts on the $\mathbb{Z}_{p}$-formal scheme $\hat{\mathcal{H}}_{p}$ [3, Chapitre I, Sect. 6] and acts on $\operatorname{Spf}\left(\mathbb{Z}_{p^{2}}\right)$ via the inverse of the arithmetic Frobenius raised to the determinant map [3, Chapitre II, Sect. 9]. Therefore, the group $\mathrm{GL}_{2}\left(\mathbb{Q}_{p}\right)$ also acts on the $\mathbb{Z}_{p^{2}}$-formal scheme $\hat{\mathcal{H}}_{p^{2}}$, its generic fiber, and on $\mathcal{G}$. The Cerednik-Drinflel'd theorem [3, Sect. 3.5.3], [24, Theorem 4.3'] implies that there are isomorphisms of $\mathbb{Q}_{p^{2}}$-rigid analytic spaces

$$
\begin{aligned}
& \Gamma_{p} \backslash \mathcal{H}_{p^{2}} \simeq \mathcal{C}_{\mathbb{Q}_{p 2}}^{\text {rig }}, \\
& \Gamma_{p} \backslash \mathcal{G}^{\text {rig }} \simeq \mathcal{A}_{\mathbb{Q}_{p^{2}}}^{\text {rig }} .
\end{aligned}
$$

The isomorphism (37) is equivariant with respect to the quaternionic actions on both sides. See also [11, Sect. 6.2], [31, Sect. 6], [29].

\subsection{Rigid analytic modular forms}

A rigid analytic function $f: \mathcal{H}_{p}\left(\mathbb{C}_{p}\right) \rightarrow \mathbb{C}_{p}$ is said to be a rigid analytic modular form of weight $k$ and level $\Gamma_{p}$ if

$$
f(\gamma z)=(c z+d)^{k} f(z)
$$

for all $z \in \mathcal{H}_{p}\left(\mathbb{C}_{p}\right)$ and $\gamma \in \Gamma_{p}$, where $\gamma(z)=(a z+b) /(c z+d)$. Denote $S_{k}^{\text {rig }}\left(\Gamma_{p}\right)$ the $\mathbb{C}_{p}$-vector space of rigid analytic modular forms of weight $k$ and level $\Gamma_{p}$. See [11, Sect. 5.2] for details.

Given a $\mathbb{Z}\left[\Gamma_{p}\right]$-module $M$, we denote $M^{\Gamma_{p}}$ the submodule consisting of $\Gamma_{p}$-invariant elements of $M$. With notation as in Sect. 4.8, consider the $\hat{\mathbb{Q}}_{p}^{\text {unr }}$-subspace $w_{\mathcal{G}}^{\Gamma_{p}}$ of $w_{\mathcal{G}}$ consisting 
of global sections which are invariant for the $\Gamma_{p}$-action. In particular, $w_{\mathcal{G}}^{\Gamma_{p}}$ is a $\mathcal{A}^{\Gamma_{p}}$-module. Given $f \in S_{k}^{\text {rig }}\left(\Gamma_{p}\right)$, define $\omega_{f}=f(z) \otimes d \tau^{\otimes k}$ in $w_{\mathcal{G}, \mathbb{C}_{p}}=w_{\mathcal{G}} \otimes_{\hat{\mathbb{Q}}_{p}^{\text {unr }}} \mathbb{C}_{p}$. The correspondence $f \mapsto \omega_{f}$ sets up a $\mathbb{C}_{p}$-linear isomorphism between $S_{k}^{\text {rig }}\left(\Gamma_{p}\right)$ and $w_{\mathcal{G}, \mathbb{C}_{p}}^{\Gamma_{p}}$.

For any sheaf $\mathcal{F}$ on $\mathcal{H}_{p}^{\text {unr }}$, denote $\mathcal{F}^{\Gamma_{p}}$ the sheaf on $\Gamma_{p} \backslash \mathcal{H}_{p}^{\text {unr }}$ defined by taking $\Gamma_{p}$-invariant sections. Also, recall the sheaves $\underline{\omega}_{\hat{\mathbb{Q}}_{p}^{\text {unr }}}$ and $\mathcal{L}_{\hat{\mathbb{Q}}_{p}^{\text {unr }}}$ introduced in (8) and (9). It is easy to see that (36) and (37) induce isomorphisms $\left(\underline{\omega}_{\mathcal{G}}^{0}\right)^{\Gamma_{p}} \simeq \underline{\omega}_{\hat{\mathbb{Q}}_{p}^{\text {unr }}}^{\text {rig }}$ and $\left(\mathcal{L}_{\mathcal{G}}^{0}\right)^{\Gamma_{p}} \simeq \mathcal{L}_{\widehat{\mathbb{Q}}_{p}^{\text {unr }}}^{\text {rig }}$ of sheaves. Combining this with the isomorphism between $S_{k}^{\text {rig }}\left(\Gamma_{p}\right)$ and $w_{\mathcal{G}, \mathbb{C}_{p}}^{\Gamma_{p}}$, we obtain a canonical isomorphism of $\mathbb{C}_{p}$-vector spaces

$$
S_{k}^{\text {rig }}\left(\Gamma_{p}\right) \simeq H^{0}\left(\mathcal{C}_{\mathbb{C}_{p}}, \underline{\omega}_{\mathbb{C}_{p}}^{\otimes k}\right) .
$$

If $E$ is a subfield of $\mathbb{C}_{p}$ containing $\mathbb{Q}_{p^{2}}$, we denote $S_{k}^{\text {rig }}\left(\Gamma_{p}, E\right)$ the $E$-subspace of $S_{k}^{\text {rig }}\left(\Gamma_{p}\right)$ consisting of those rigid analytic functions which are defined over $E$. Using the CerednikDrinfel'd theorem (36) it is easy to see that (38) induces an isomorphism of $E$-rigid analytic spaces

$$
S_{k}^{\mathrm{rig}}(\Gamma, F) \simeq S_{k}^{\mathrm{alg}}\left(\Gamma_{p}, F\right) .
$$

\subsection{The $p$-adic Maass-Shimura operator}

Taking $\Gamma_{p}$-invariants defines a map, for integers $k \geq 0$ and $j \geq 0$

$$
\Theta_{p, k}^{j}:\left(w_{\mathcal{G}, n}^{*}\right)^{\Gamma_{p}} \longrightarrow\left(w_{\mathcal{G}, n+2 j}^{*}\right)^{\Gamma_{p}}
$$

where recall that $\Theta_{p, k}^{j}$ was introduced in (32), and we understand that $\Theta_{p, k}^{0}=\Theta_{p, k}$,

An alternative way to introduce $\Theta_{p, k}^{j}$ is the following. Recall the operator $\tilde{\nabla}_{n}$ in (11) and, for any integer $j \geq 0$, define $\tilde{\nabla}_{n}^{j}: \mathcal{L}_{\hat{\mathbb{Q}}_{p}^{\text {unr }}, n} \rightarrow \mathcal{L}_{\hat{\mathbb{Q}}_{p}^{\text {unr }}, n+2 j}$ by the formula

$$
\tilde{\nabla}_{n}^{j}=\tilde{\nabla}_{n+2(j-1)} \circ \cdots \circ \tilde{\nabla}_{n+2} \circ \tilde{\nabla}_{n} .
$$

Considering the associated rigid analytic sheaves, and taking global sections, we obtain a map of $\mathcal{A}$-modules $\tilde{\nabla}_{n}^{j, \text { rig }}: \Lambda_{\mathcal{G}, n}^{\Gamma_{p}} \rightarrow \Lambda_{\mathcal{G}, n+2 j}^{\Gamma_{p}}$. One may define the operator

$$
\Theta_{p, n}^{j}: w_{\mathcal{G}, n}^{\Gamma_{p}} \stackrel{i_{\mathcal{G}, n}}{\longrightarrow} \Lambda_{\mathcal{G}, n}^{\Gamma_{p}} \stackrel{\tilde{\nabla}_{n}^{j, \text { rig }}}{\longrightarrow} \Lambda_{\mathcal{G}, n+2 j}^{\Gamma_{p}} \longrightarrow\left(\Lambda_{\mathcal{G}, n+2 j}^{*}\right)^{\Gamma_{p}} \stackrel{\Psi_{p, n+2 j}}{\longrightarrow}\left(w_{\mathcal{G}, n+2 j}^{*}\right)^{\Gamma_{p}}
$$

By (24), $d \tau^{*}$ is horizontal for $\nabla_{\mathcal{G}}^{*}$, and therefore $\Theta_{p, n}^{j}$ coincides with the restriction of $\Theta_{p, k}^{j}$ to $w_{\mathcal{G}, n}^{\Gamma_{p}}$, thus justifying the abuse of notation.

\subsection{Comparison of Maass-Shimura operators at CM points}

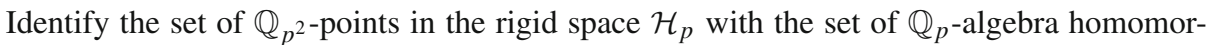
phisms $\operatorname{Hom}\left(\mathbb{Q}_{p^{2}}, \mathrm{M}_{2}\left(\mathbb{Q}_{p}\right)\right)$ as follows: any $\Psi \in \operatorname{Hom}\left(\mathbb{Q}_{p^{2}}, \mathbf{M}_{2}\left(\mathbb{Q}_{p}\right)\right)$ defines an action of $\mathbb{Q}_{p^{2}}^{\times}$on $\mathcal{H}_{p}\left(\mathbb{Q}_{p^{2}}\right)=\mathbb{Q}_{p^{2}}-\mathbb{Q}_{p}$ by fractional linear transformations, and the point $z \in \mathcal{H}_{p}\left(\mathbb{Q}_{p^{2}}\right)$ associated with $\Psi$ is characterised by the property $\Psi(a)\left(\begin{array}{l}z \\ 1\end{array}\right)=a\left(\begin{array}{l}z \\ 1\end{array}\right)$, for all $a \in \mathbb{Q}_{p^{2}}^{\times}$. 
Given a representation $\rho=\left(\rho_{1}, \rho_{2}\right): \mathrm{GL}_{2} \times \mathrm{GL}_{2} \rightarrow \mathrm{GL}(V)$, the stalk $\mathcal{E}(V)_{\Psi}$ of $\mathcal{E}(V)$ at a point $\Psi \in \operatorname{Hom}\left(\mathbb{Q}_{p^{2}}, \mathrm{M}_{2}\left(\mathbb{Q}_{p}\right)\right)$ can be described explicitly. One first observes that the structure of filtered convergent $F$-isocrystal of $\mathcal{E}(V)$ induces a structure of filtered Frobenius module on the fiber $\mathcal{E}(V)_{\Psi}$. On the other hand, one attaches to such a pair $(V, \Psi)$ a filtered Frobenius module $V_{\Psi}$ whose underlying vector space is $V_{\mathbb{Q}_{p}^{\text {unr }}}=V \otimes_{\mathbb{Q}_{p}} \mathbb{Q}_{p}^{\text {unr }}$ (see [23, Sect. 4.2] for details). Denote $F^{\bullet} V_{\Psi}$ the filtration of the filtered Frobenius module $V_{\Psi}$; this is then a filtration on $V_{\mathbb{Q}_{p}^{u n r}}$ which depends on $\Psi$. Let $\operatorname{gr}^{i}\left(F^{\bullet} V_{\Psi}\right)=F^{i} V_{\Psi} / F^{i-1} V_{\Psi}$ be the graded pieces of the filtration. If $V$ is pure of weight $n$, we have an isomorphism $\operatorname{gr}^{i}\left(F^{\bullet} V_{\Psi}\right) \simeq V_{i}$ (where $V_{j}$ is be the subspace of $V_{\mathbb{Q}_{p}^{\text {unr }}}$ consisting of elements $v$ satisfying the property $\rho(\Psi(a))(v)=a^{j} \sigma(a)^{n-j} v$ for all $\left.a \in \mathbb{Q}_{p^{2}}\right)$ as well as a decomposition $V_{\mathbb{Q}_{p}^{u n r}}=$ $\bigoplus_{i \in \mathbb{Z}} \operatorname{gr}^{i}\left(F^{\bullet} V_{\Psi}\right)$. For $\Psi \in \operatorname{Hom}\left(\mathbb{Q}_{p^{2}}, \mathrm{M}_{2}\left(\mathbb{Q}_{p}\right)\right)$, denote $\bar{\Psi}$ the morphism of $\mathbb{Q}_{p}$-algebras obtained by composition $\Psi$ with the main involution of $\mathrm{M}_{2}\left(\mathbb{Q}_{p}\right)$; therefore, if $\Psi(x)=\left(\begin{array}{ll}a & b \\ c & d\end{array}\right)$ then $\bar{\Psi}(x)=\left(\begin{array}{cc}d & -b \\ -c & a\end{array}\right)$. If $V$ is pure of weight $n$, then the graduate pieces $\operatorname{gr}^{i}\left(F^{\bullet} V_{\Psi}\right)$ and $\operatorname{gr}^{n-i}\left(F^{\bullet} V_{\bar{\Psi}}\right)$ are equal, for all $i \in \mathbb{Z}$. In particular, for $V=\mathrm{M}_{2}$ we have

$$
\operatorname{gr}^{1}\left(F^{\bullet}\left(\mathrm{M}_{2}\right) \Psi\right) \simeq \operatorname{gr}^{2}\left(F^{\bullet}\left(\mathrm{M}_{2}\right)_{\bar{\Psi}}\right) .
$$

and therefore there is an exact sequence:

$$
0 \longrightarrow \operatorname{gr}^{1}\left(F^{\bullet}\left(\mathrm{M}_{2}\right)_{\Psi}\right) \longrightarrow\left(\mathrm{M}_{2}\right)_{\Psi} \longrightarrow \operatorname{gr}^{1}\left(F^{\bullet}\left(\mathrm{M}_{2}\right)_{\bar{\Psi}}\right) \longrightarrow 0
$$

and a canonical decomposition

$$
\left(\mathrm{M}_{2}\right)_{\Psi} \simeq \operatorname{gr}^{1}\left(F^{\bullet}\left(\mathrm{M}_{2}\right)_{\Psi}\right) \bigoplus \operatorname{gr}^{1}\left(F^{\bullet}\left(\mathrm{M}_{2}\right)_{\bar{\Psi}}\right)
$$

One can choose generators $\omega_{1}, \omega_{2}$ of the $\hat{\mathbb{Q}}_{p}^{\text {unr }}$-vector space $\operatorname{gr}^{1}\left(F^{\bullet}\left(\mathrm{M}_{2}\right)_{\Psi}\right)$ so that $\omega_{1}$ and $\omega_{2}$ are defined over $\mathbb{Q}_{p^{2}}$. Then $\bar{\omega}_{1}$ and $\bar{\omega}_{2}$ are generators of the $\hat{\mathbb{Q}}_{p}^{\mathrm{unr}}$-vector space gr ${ }^{1}\left(F^{\bullet}\left(\mathrm{M}_{2}\right)_{\bar{\Psi}}\right)$, where $\omega_{i} \mapsto \bar{\omega}_{i}$ for $i=1,2$ denotes the action of $\operatorname{Gal}\left(\mathbb{Q}_{p^{2}} / \mathbb{Q}_{p}\right)$ on $\omega_{i}$. It follows that the Hodge splitting coincides on quadratic points with the projection $\left(\mathrm{M}_{2}\right)_{\Psi} \rightarrow \operatorname{gr}^{1}\left(F^{\bullet}\left(\mathrm{M}_{2}\right)_{\Psi}\right)$ to the first factor in the decomposition (41).

We now apply the above results to the situation of the previous sections. Recall that $K$ is a imaginary quadratic field and $f \in H^{0}\left(\mathcal{C}_{\mathbb{Q}}, \underline{\omega}_{\mathbb{Q}}^{\otimes k}\right)$ is an algebraic modular form of weight $k$ and level $N^{+} N^{-} p$ with $p \nmid N=N^{+} N^{-}$and $\left(N^{+}, N^{-}\right)=1$. We write $f_{\infty}: \mathcal{H}_{p} \rightarrow \mathbb{C}$ and $f_{p}: \mathcal{H}_{p} \rightarrow \mathbb{C}_{p}$ for the holomorphic and the rigid analytic modular forms corresponding to $f$, respectively. Assume $N^{-} p$ is a product of an even number of distinct primes, each of them inert in $K$, and that all primes dividing $N^{+}$are split in $K$. Let $P \in \mathcal{C}_{\mathbb{Q}}(K)$ be a Heegner point, and assume that $P \in \mathcal{C}_{\mathbb{C}}(\mathbb{C})$ is represented by the point $\tau_{\infty} \in \mathcal{H}_{\infty}$ modulo $\Gamma_{\infty}$, while $P \in \mathcal{C}_{\mathbb{C}_{p}}\left(\mathbb{C}_{p}\right)$ is represented by the point $\tau_{p} \in \mathcal{H}_{p}$ modulo $\Gamma_{p}$. Fix embeddings $\overline{\mathbb{Q}} \hookrightarrow \overline{\mathbb{Q}}_{p}$ and $\overline{\mathbb{Q}} \hookrightarrow \mathbb{C}_{p}$ which allows us to view algebraic numbers as complex and $p$-adic numbers.

Theorem 5.1 For any positive integer $j$ we have the equality

$$
\Theta_{\infty, k}^{j}\left(f_{\infty}\right)\left(\tau_{\infty}\right)=\Theta_{p, k}^{j}\left(f_{p}\right)\left(\tau_{p}\right) .
$$

Proof We mimic a well known argument of Katz when $p$ is split in $K$ ([26, Theorems 2.4.5, 2.4.7]; see also [4, Proposition 1.12], [19, Theorem 3.5], [37, Proposition 2.12]). Let $A_{P}$ be the false elliptic curve corresponding to the Heegner point $P$. The algebraic $\mathrm{CM}$ splitting of $A_{P}$ coincides both with the Hodge splitting and the $p$-adic splitting, and therefore the values of $\Psi_{\infty, n}$ and $\Psi_{p, n}$ at CM points are the same. Since the construction of the Maass-Shimura operators is algebraic, we see that $\nabla_{n}^{\mathrm{ran}}\left(f_{\infty}\right)$ coincides with $\nabla_{n}^{\mathrm{rig}}\left(f_{p}\right)$, and the same still holds 
for the iterates of the Maass-Shimura operator, which also admit an algebraic construction. The result follows.

\subsection{Nearly rigid analytic modular forms}

In this subsection we make explicit the relation between the results of this paper and those of Franc's thesis [15]; it is independent from the rest of the paper.

We first introduce a $\mathbb{C}_{p}$-subspace of the $\mathbb{C}_{p}$-vector space $\mathcal{C}^{0}$ of continuous functions, which plays a role analogue to that of nearly holomorphic functions in the real analytic setting. For this part, we closely follow [15]. The assignment $X \mapsto 1 /\left(z-z^{*}\right)$ defines an injective homomorphism $\mathcal{A}[X] \hookrightarrow \mathcal{C}[15$, Proposition 4.3.3]. Define the $\mathcal{A}$-algebra $\mathcal{N}$ of nearly rigid analytic functions to be the image of this map ( $c f$. [15, Definition 4.3.5]). By definition, $\mathcal{N}$ is a sub- $\mathcal{A}$-algebra of $\mathcal{A}^{*}$. The $\mathcal{A}$-algebra $\mathcal{N}$ is equipped with a canonical graduation $\mathcal{N}=\bigoplus_{j \geq 0} \mathcal{N}^{(j)}$ where for each integer $j \geq 0$, we denote $\mathcal{N}^{(j)}$ the sub- $\mathcal{A}$-algebra of $\mathcal{N}$ consisting of functions $f$ which can be written in the form

$$
f(z)=\sum_{i=0}^{j} \frac{f_{i}(z)}{(z-\sigma(z))^{i}}
$$

with $f_{i} \in \mathcal{A}$. The Maass-Shimura operator $\delta_{p, k}$ restricts to an operator (denoted with the same symbol) $\delta_{p, k}: \mathcal{N} \rightarrow \mathcal{N}$ which takes $\mathcal{N}^{(j)}$ to $\mathcal{N}^{(j+2)}$.

Define now $\mathcal{N}_{k}\left(\Gamma_{p}\right)=\mathcal{N}_{k}^{\Gamma_{p}}$ to be the $\mathbb{C}_{p}$-subalgebra of $\mathcal{N}$ consisting of functions which are invariant under the weight $k$ action of $\Gamma_{p}$ on $\mathcal{N}$, i.e. those functions satisfying the transformation property $f(\gamma z)=(c z+d)^{k} f(z)$ for all $z \in \hat{\mathbb{Q}}_{p}^{\text {unr }}-\mathbb{Q}_{p}$ and $\gamma \in \Gamma_{p}$. Note that $S_{k}^{\text {rig }}\left(\Gamma_{p}\right) \subseteq \mathcal{N}_{k}\left(\Gamma_{p}\right)$. We call $\mathcal{N}_{k}\left(\Gamma_{p}\right)$ the $\mathbb{C}_{p}$-vector space of nearly rigid analytic modular forms of weight $k$ and level $\Gamma_{p}$. Define also $\mathcal{N}_{k}^{(j)}\left(\Gamma_{p}\right)=\mathcal{N}_{k}\left(\Gamma_{p}\right) \cap \mathcal{N}^{(j)}$. The operator $\delta_{p, k}^{j}$ introduced in Sect. 4.7 restricts to a map $\delta_{p, k}^{j}: \mathcal{N}_{k}\left(\Gamma_{p}\right) \rightarrow \mathcal{N}_{k+2 j}\left(\Gamma_{p}\right)$ [15, Lemma 4.3.8]. By [15, Theorem 4.3.11], for each integer $r \geq 0$ we have an isomorphism of $\mathbb{C}_{p}$-vector spaces

$$
\bigoplus_{j=0}^{r} S_{k+2(r-j)}^{\mathrm{rig}}\left(\Gamma_{p}\right) \simeq \mathcal{N}_{p, k+2 r}^{(r)}\left(\Gamma_{p}\right)
$$

which maps $\left(h_{j}\right)_{j=0}^{k+2(r-j)}$ to $\sum_{j=0}^{k+2(r-j)} \delta_{p, k}^{j}\left(h_{j}\right)$.

Corollary 5.2 (Franc) Let $\tau_{p} \in \mathcal{H}_{p}$ corresponds to a Heegner point. The values $\frac{\delta_{p, k}^{j}(f)\left(\tau_{p}\right)}{t_{p}^{j}}$ are algebraic for each integer $j \geq 0$.

Proof The result is clear from Theorem 5.1 since this is known for $\Theta_{\infty, k}^{j}(f)\left(\tau_{\infty}\right)$.

Remark 5.3 Equation (35) answers affirmatively one of the questions left in [15, Sect. 6.1] whether if it was possible to describe the $p$-adic Maass-Shimura operator $\delta_{p, k}^{j}$ introduced in [15] in a more conceptual way, similar to that in the complex case. Corollary 5.2 is the main result of [15], which was obtained via a completely different method, following more closely the complex analytic approach of Shimura. 


\section{The Coleman primitive}

Write $\nabla=\nabla_{\mathcal{G}, n}^{*}, \nabla^{1,0}=\nabla_{\mathcal{G}}^{1,0}, \nabla^{0,1}=\nabla_{\mathcal{G}}^{0,1}$ and $\langle\rangle=,\langle,\rangle_{\mathcal{G}, n}^{*}$ to simplify the notation. For any $n$ and any $j$, whenever there is not possible confusion, we write $\Theta_{p}=\Theta_{p, n}$ and $\Theta_{p, n}^{j}=\Theta_{p}^{j}$ for the $p$-adic Maass-Shimura operator, and $\Psi_{p}=\Psi_{p, n}$ for the splitting of the Hodge filtration.

We set up the notation $\omega_{\text {can }}=d \tau$ and $\eta_{\text {can }}=\frac{d \tau^{*}}{z^{*}-z}$. Since $\langle d x, d y\rangle=-1$, we have $\left\langle\omega_{\text {can }}, \eta_{\text {can }}\right\rangle=1$. We also write $\omega_{\text {can }}^{j} \eta_{\text {can }}^{n-j}=\omega_{\text {can }}^{\otimes j} \otimes \eta_{\text {can }}^{\otimes n-j}$.

The computation of the Gauss-Manin connection gives

$$
\begin{aligned}
& \nabla\left(\omega_{\text {can }}\right)=\left(-\frac{\omega_{\text {can }}}{z^{*}-z}+\eta_{\text {can }}\right) \otimes d z, \\
& \nabla\left(\eta_{\text {can }}\right)=-\frac{\omega_{\text {can }} \otimes d z^{*}}{\left(z^{*}-z\right)^{2}}+\frac{\eta_{\text {can }} \otimes d z}{z^{*}-z} .
\end{aligned}
$$

Let $f: \mathcal{H}_{p} \rightarrow \mathbb{C}_{p}$ be a rigid modular form giving rise to a section $\omega_{f}=f(z) \otimes d \tau^{\otimes k}$. Put $n=k-2$. Using the Kodaira-Spencer map, we identify this with $\omega_{f}=f(z) d z \otimes d \tau^{\otimes n}$. Let $F_{f}$ be the Coleman primitive of the differential form $\omega_{f}$, satisfying the differential equation

$$
\nabla\left(F_{f}\right)=\omega_{f} .
$$

Define for $j=n / 2, \ldots, n$ an integer

$$
G_{j}(z)=\left\langle F_{f}(z), \omega_{\text {can }}^{j} \eta_{\text {can }}^{n-j}\right\rangle \otimes \omega_{\text {can }}^{n-2 j} .
$$

Theorem 6.1 $\Theta_{p}^{j+1}\left(G_{j}\right)=j ! \omega_{f}$.

Proof This result, which is proved by means of a simple and explicit computation, is the analogue of [4, Proposition 3.24] (and also of [19, Theorem 7.3]), but we provide a complete proof since our formalism is quite different from that in [4], where one can use the Tate curve and the $q$-expansion principle. As in loc. cit. we show that $\Theta_{p}\left(G_{0}(z)\right)=\omega_{f}$ and $\Theta_{p}\left(G_{j}(z)\right)=j G_{j-1}(z)$.

We first compute $\nabla\left(G_{0}(z)\right)$. We have:

$$
\begin{aligned}
\nabla & \left(G_{0}(z)\right) \\
& =\nabla\left(\left\langle F_{f}(z), \eta_{\mathrm{can}}^{n}\right\rangle \otimes \omega_{\mathrm{can}}^{n}\right) \\
& =\left\langle\nabla\left(F_{f}(z)\right), \eta_{\mathrm{can}}^{n}\right\rangle \otimes \omega_{\mathrm{can}}^{n}+\left\langle F_{f}(z), \nabla\left(\eta_{\mathrm{can}}^{n}\right)\right\rangle \otimes \omega_{\mathrm{can}}^{n}+\left\langle F_{f}(z), \eta_{\mathrm{can}}^{n}\right\rangle \otimes \nabla\left(\omega_{\mathrm{can}}^{n}\right) \\
& =\left\langle f(z) d z \otimes \omega_{\mathrm{can}}^{n}, \eta_{\mathrm{can}}^{n}\right\rangle \otimes \omega_{\mathrm{can}}^{n}+\left\langle F_{f}(z), \nabla\left(\eta_{\mathrm{can}}^{n}\right)\right\rangle \otimes \omega_{\mathrm{can}}^{n}+\left\langle F_{f}(z), \eta_{\mathrm{can}}^{n}\right\rangle \otimes \nabla\left(\omega_{\mathrm{can}}^{n}\right) .
\end{aligned}
$$

We now compute the last two pieces:

$$
\begin{aligned}
\left\langle F_{f}(z), \nabla\left(\eta_{\mathrm{can}}^{n}\right)\right\rangle \otimes \omega_{\mathrm{can}}^{n} \\
\quad=\left\langle F_{f}(z), n \eta_{\mathrm{can}}^{n-1} \nabla\left(\eta_{\mathrm{can}}\right)\right\rangle \otimes \omega_{\mathrm{can}}^{n} \\
\quad=\left\langle F_{f}(z), n \eta_{\mathrm{can}}^{n-1}\left(\frac{-\omega_{\mathrm{can}} \otimes d z^{*}}{\left(z^{*}-z\right)^{2}}+\frac{\eta_{\mathrm{can}} \otimes d z}{z^{*}-z}\right)\right\rangle \otimes \omega_{\mathrm{can}}^{n} \\
\quad=-\left\langle F_{f}(z), \frac{n \eta_{\mathrm{can}}^{n-1} \omega_{\mathrm{can}} \otimes d z^{*}}{\left(z^{*}-z\right)^{2}}\right\rangle \otimes \omega_{\mathrm{can}}^{n}+\left\langle F_{f}(z), \frac{n \eta_{\mathrm{can}}^{n} \otimes d z}{z^{*}-z}\right\rangle \otimes \omega_{\mathrm{can}}^{n} \\
\quad=-\left\langle F_{f}(z), \eta_{\mathrm{can}}^{n-1} \omega_{\mathrm{can}}\right\rangle \otimes \frac{n \omega_{\mathrm{can}}^{n} \otimes d z^{*}}{\left(z^{*}-z\right)^{2}}+\left\langle F_{f}(z), \eta_{\mathrm{can}}^{n}\right\rangle \otimes \frac{n \omega_{\mathrm{can}}^{n} \otimes d z}{z^{*}-z}
\end{aligned}
$$


and

$$
\begin{aligned}
& \left\langle F_{f}(z), \eta_{\mathrm{can}}^{n}\right\rangle \otimes \nabla\left(\omega_{\mathrm{can}}^{n}\right) \\
& \quad=\left\langle F_{f}(z), \eta_{\mathrm{can}}^{n}\right\rangle \otimes \nabla\left(\omega_{\mathrm{can}}^{n}\right) \\
& =\left\langle F_{f}(z), \eta_{\mathrm{can}}^{n}\right\rangle \otimes n \omega_{\mathrm{can}}^{n-1} \nabla\left(\omega_{\mathrm{can}}\right) \\
& =\left\langle F_{f}(z), \eta_{\mathrm{can}}^{n}\right\rangle \otimes n \omega_{\mathrm{can}}^{n-1}\left(-\frac{\omega_{\mathrm{can}}}{z^{*}-z}+\eta_{\mathrm{can}}\right) \otimes d z \\
& =-\left\langle F_{f}(z), \eta_{\mathrm{can}}^{n}\right\rangle \otimes \frac{n \omega_{\mathrm{can}}^{n} \otimes d z}{z^{*}-z}+\left\langle F_{f}(z), \eta_{\mathrm{can}}^{n}\right\rangle \otimes n \omega_{\mathrm{can}}^{n-1} \eta_{\mathrm{can}} \otimes d z .
\end{aligned}
$$

Therefore the sum of these two pieces gives:

$$
-\left\langle F_{f}(z), \eta_{\mathrm{can}}^{n-1} \omega_{\mathrm{can}}\right\rangle \otimes \frac{n \omega_{\mathrm{can}}^{n} \otimes d z^{*}}{\left(z-z^{*}\right)^{2}}+\left\langle F_{f}(z), \eta_{\mathrm{can}}^{n}\right\rangle \otimes n \omega_{\mathrm{can}}^{n-1} \eta_{\mathrm{can}} \otimes d z
$$

Recall now that $\Psi\left(\eta_{\text {can }}\right)=0$ and $\Psi\left(d z^{*}\right)=0$. Therefore, using the Kodaira-Spencer map to replace $d z$ with $\omega_{\text {can }}^{2}$, and applying $\Psi$ we have

$$
\Theta_{p}\left(G_{0}(z)\right)=\omega_{f}\left\langle\omega_{\text {can }}^{n}, \eta_{\text {can }}^{n}\right\rangle=\omega_{f} .
$$

We now compute $\nabla\left(G_{j}(z)\right)$ for $j \geq 1$. The Gauss-Manin connection

$$
\nabla\left(G_{j}(z)\right)=\nabla\left(\left\langle F_{f}(z), \omega_{\mathrm{can}}^{j} \eta_{\mathrm{can}}^{n-j}\right\rangle \otimes \omega_{\mathrm{can}}^{n-2 j}\right)
$$

is the sum of three terms

$$
\begin{aligned}
& \left\langle\nabla\left(F_{f}(z)\right), \omega_{\mathrm{can}}^{j} \eta_{\text {can }}^{n-j}\right\rangle \otimes \omega_{\text {can }}^{n-2 j} \\
& \quad+\left\langle F_{f}(z), \nabla\left(\omega_{\text {can }}^{j} \eta_{\text {can }}^{n-j}\right)\right\rangle \otimes \omega_{\text {can }}^{n-2 j}+\left\langle F_{f}(z), \omega_{\text {can }}^{j} \eta_{\text {can }}^{n-j}\right\rangle \otimes \nabla\left(\omega_{\text {can }}^{n-2 j}\right)
\end{aligned}
$$

which we calculate separately as before. First, since $j>0$, we have

$$
\left\langle\nabla\left(F_{f}(z)\right), \omega_{\mathrm{can}}^{j} \eta_{\mathrm{can}}^{n-j}\right\rangle \otimes \omega_{\mathrm{can}}^{n-2 j}=\left\langle f(z) d z \otimes \omega_{\mathrm{can}}^{n}, \omega_{\mathrm{can}}^{j} \eta_{\mathrm{can}}^{n-j}\right\rangle=0
$$

Next, a simple computation shows that

$$
\nabla\left(\omega_{\mathrm{can}}^{j} \eta_{\mathrm{can}}^{n-j}\right)=(n-2 j) \omega_{\mathrm{can}}^{j} \eta_{\mathrm{can}}^{n-j} \otimes \frac{d z}{z^{*}-z}+j \omega_{\mathrm{can}}^{j-1} \eta_{\mathrm{can}}^{n-j+1} \otimes d z-(n-j) \omega_{\mathrm{can}}^{j+1} \eta_{\mathrm{can}}^{n-j-1} \otimes \frac{d z^{*}}{\left(z^{*}-z\right)^{2}}
$$

and therefore the second summand in (43) is

$$
\begin{aligned}
\left\langle F_{f}(z), \nabla\left(\omega_{\mathrm{can}}^{j} \eta_{\mathrm{can}}^{n-j}\right)\right\rangle \otimes \omega_{\mathrm{can}}^{n-2 j}= & (n-2 j)\left\langle F_{f}(z), \omega_{\mathrm{can}}^{j} \eta_{\mathrm{can}}^{n-j}\right\rangle \otimes \omega_{\mathrm{can}}^{n-2 j} \otimes \frac{d z}{z-z^{*}} \\
& -j\left\langle F_{f}(z), \omega_{\mathrm{can}}^{j-1} \eta_{\mathrm{can}}^{n-j+1}\right\rangle \otimes \omega_{\mathrm{can}}^{n-2 j} \otimes d z+ \\
& -(n-j)\left\langle F_{f}(z), \omega_{\mathrm{can}}^{j+1} \eta_{\mathrm{can}}^{n-j-1}\right\rangle \otimes \omega_{\mathrm{can}}^{n-2 j} \otimes \frac{d z^{*}}{\left(z-z^{*}\right)^{2}}
\end{aligned}
$$


Finally, the third summand is

$$
\begin{aligned}
& \left\langle F_{f}(z), \omega_{\mathrm{can}}^{j} \eta_{\mathrm{can}}^{n-j}\right\rangle \otimes \nabla\left(\omega_{\mathrm{can}}^{n-2 j}\right) \\
& =(n-2 j)\left\langle F_{f}(z), \omega_{\mathrm{can}}^{j} \eta_{\mathrm{can}}^{n-j}\right\rangle \otimes \omega_{\mathrm{can}}^{n-2 j-1} \nabla\left(\omega_{\mathrm{can}}\right) \\
& =(n-2 j)\left\langle F_{f}(z), \omega_{\mathrm{can}}^{j} \eta_{\mathrm{can}}^{n-j}\right\rangle \otimes \omega_{\mathrm{can}}^{n-2 j-1}\left(-\frac{\omega_{\mathrm{can}}}{z-z^{*}}+\eta_{\mathrm{can}}\right) \otimes d z \\
& =-(n-2 j)\left\langle F_{f}(z), \omega_{\mathrm{can}}^{j} \eta_{\mathrm{can}}^{n-j}\right\rangle \otimes \omega_{\mathrm{can}}^{n-2 j} \otimes \frac{d z}{z-z^{*}}+ \\
& \quad+(n-2 j)\left\langle F_{f}(z), \omega_{\mathrm{can}}^{j} \eta_{\mathrm{can}}^{n-j}\right\rangle \otimes \omega_{\mathrm{can}}^{n-2 j-1} \eta_{\mathrm{can}} \otimes d z .
\end{aligned}
$$

Summing up the pieces in (43), using the Kodaira-Spencer map to replace $d z$ with $\omega_{\text {can }}^{2}$, and applying the splitting of the Hodge filtration $\Psi$ which kills $\eta_{\text {can }}$ and $d z^{*}$, we have

$$
\Theta_{p}\left(G_{j}(z)\right)=j\left\langle F_{f}(z), \omega_{\mathrm{can}}^{j-1} \eta_{\mathrm{can}}^{n-(j-1)}\right\rangle \otimes \omega_{\mathrm{can}}^{n-2(j-1)}=j G_{j-1}(z) .
$$

The result follows.

\section{Generalised Heegner cycles and $p$-adic Maass-Shimura operator}

The aim of this section is to prove Theorem 1.5 stated in the Introduction, which relates generalised Heegner cycles and the $p$-adic Maass-Shimura operator.

\subsection{The generalised Kuga-Sato motive}

Fix an even integer $k \geq 2$ and put $n=k-2, m=n / 2$. Let $A_{0}$ be a false elliptic curve with quaternionic multiplication and full level- $M$ structure, defined over $H$ (the Hilbert class field of $K$ ) and with complex multiplication by $\mathcal{O}_{K}$; the action of $\mathcal{O}_{K}$ is required to commute with the quaternionic action, and this implies that $A_{0}$ is isogenous to $E \times E$ for an elliptic curve $E$ with $\mathrm{CM}$ by $\mathcal{O}_{K}$. Fix a field $F \supset H$ and consider the $(2 n+1)$-dimensional variety $X_{m}$ over $F$ given by

$$
X_{m}:=\mathcal{A}^{m} \times A_{0}^{m} .
$$

Here and in the following we simplify the notation and simply write $\mathcal{A}, \mathcal{C}$ and $A_{0}$ for $\mathcal{A}_{F}$, $\mathcal{C}_{F}$ and $\left(A_{0}\right)_{F}$, unless we need to stress the field of definition in which case we keep the full notation. The variety $X_{m}$ is equipped with a proper morphism $\pi: X_{m} \rightarrow \mathcal{C}$ with $2 n$ dimensional fibers. The fibers above points of $\mathcal{C}$ are products of the form $A^{m} \times A_{0}^{m}$.

The de Rham cohomology of $\mathcal{C}$ attached to $\mathcal{L}_{n}$, denoted $H_{\mathrm{dR}}^{1}\left(\mathcal{C}, \mathcal{L}_{n}, \nabla\right)$, is defined to be the 1-st hypercohomology of the complex

$$
0 \longrightarrow \mathcal{L}_{n} \stackrel{\nabla}{\longrightarrow} \mathcal{L}_{n} \otimes \Omega_{\mathcal{C}}^{1} \longrightarrow 0 .
$$

As shown in [19, Corollary 6.3], one can define a projector $\epsilon_{\mathcal{A}}$ (denoted $P$ in loc. cit.) in the ring of correspondences $\operatorname{Corr}_{\mathcal{C}}\left(\mathcal{A}^{m}, \mathcal{A}^{m}\right)$, such that

$$
\begin{aligned}
& \epsilon_{\mathcal{A}} H_{\mathrm{dR}}^{*}\left(\mathcal{A}_{m} / F\right) \subseteq H_{\mathrm{dR}}^{n+1}\left(\mathcal{A}_{m} / F\right), \\
& \epsilon_{\mathcal{A}} H_{\mathrm{dR}}^{*}\left(\mathcal{A}_{m} / F\right) \simeq H_{\mathrm{dR}}^{1}\left(\mathcal{C}, \mathcal{L}_{n}, \nabla\right) .
\end{aligned}
$$


On the other hand, by [19, Proposition 6.4], we can define a projector $\epsilon_{A} \in \operatorname{Corr}\left(A_{0}^{m}, A_{0}^{m}\right)$ (which is defined by means of $\epsilon_{\mathcal{A}}$ ) such that

$$
\epsilon_{A_{0}} H_{\mathrm{dR}}^{*}\left(A_{0}^{m} / F\right)=\operatorname{Sym}^{n} e H_{\mathrm{dR}}^{1}\left(A_{0} / F\right) .
$$

The projectors $\epsilon_{\mathcal{A}}$ and $\epsilon_{A}$ are commuting idempotents when viewed in the $\operatorname{ring} \operatorname{Corr}_{\mathcal{C}}\left(X_{m}\right.$, $\left.X_{m}\right)$. We define $\epsilon=\epsilon_{\mathcal{A}} \epsilon_{A_{0}}$ and denote $\mathcal{D}$ the motive $\left(X_{m}, \epsilon\right)$. By [19, Proposition 6.5] and (44), (45), (46) we see that

$$
\epsilon H_{\mathrm{dR}}^{i}\left(X_{m} / F\right)=\left\{\begin{array}{l}
H_{\mathrm{dR}}^{1}\left(\mathcal{C}, \mathcal{L}_{n}, \nabla\right) \otimes \operatorname{Sym}^{n} e H_{\mathrm{dR}}^{1}\left(A_{0}^{m} / F\right), \text { if } i=2 n+1, \\
0, \text { if } i \neq 2 n+1 .
\end{array}\right.
$$

Let $v$ be the place of $F$ above $p$ induced by the inclusion $F \subseteq \overline{\mathbb{Q}} \hookrightarrow \mathbb{C}_{p}$, which for simplicity we assume to be unramified over $p$. Using the explicit description (47) of the Hodge filtration, one can see that the $p$-adic Abel-Jacobi map for the nullhomologous $(n+1)$-th Chow cycles of the motive $\mathcal{D}$ can be viewed as a map

$$
\mathrm{AJ}_{p}: \mathrm{CH}_{0}^{n+1}(\mathcal{D})\left(F_{v}\right) \longrightarrow\left(S_{k}^{\mathrm{rig}}\left(\Gamma_{p}, F_{v}\right) \otimes_{F_{v}} \operatorname{Sym}^{n} e H_{\mathrm{dR}}^{1}\left(A / F_{v}\right)\right)^{\vee} .
$$

Here $(\cdot)^{\vee}$ denotes the $F_{v}$-linear dual. For details, see [23, page 362] and [30, Sect. 4.2]; see also [32] and [33].

\subsection{Generalized Heegner cycles}

Let $\varphi: A_{0} \rightarrow A$ be an isogeny (defined over $\bar{K}$ ) of false elliptic curves, of degree $d_{\varphi}$ prime to $N p$. Let $P_{A}$ be the point on $\mathcal{C}$ corresponding to $A$ with level structure given by composing $\varphi$ with the level structure of $A_{0}$. We associate to any pair $(\varphi, A)$ a codimension $n+1$ cycle $\Upsilon_{\varphi}$ on $X_{m}$ by defining

$$
\Upsilon_{\varphi}:=\left(\Gamma_{\varphi}\right)^{m} \subset\left(A \times A_{0}\right)^{m}
$$

where $\Gamma_{\varphi}=\left\{(\varphi(x), x): x \in A_{0}\right\} \subset A \times A_{0}$ is the graph of $\varphi$. We then set

$$
\Delta_{\varphi}:=\epsilon \Upsilon_{\varphi} .
$$

The cycle $\Delta_{\varphi}$ of $\mathcal{D}$ is supported on the fiber above $P_{A}$ and has codimension $n+1$ in $\mathcal{A}^{m} \times A_{0}^{m}$, thus $\Delta_{\varphi} \in \mathrm{CH}^{n+1}(\mathcal{D})$. By (47), the cycle $\Delta_{\varphi}$ is homologous to zero. See [30] for details.

We now compute the image of $\Delta_{\varphi}$ under the Abel-Jacobi map. The de Rham cohomology group $H_{\mathrm{dR}}^{1}(A / F)$ of a false elliptic curve $A$ defined over a field $F$ containing the Hilbert class field $H$ of $K$ is equipped with the Poincaré pairing $\langle,\rangle_{H_{\mathrm{dR}}^{1}(A / F)}$, which we simply denote $\langle,\rangle_{A}$. Recall the canonical differentials $\omega_{\text {can }}, \eta_{\text {can }}$ introduced in Sect. 6; taking the fiber at $A_{0}$, the universal differential $\omega_{\text {can }}$ defines a differential $\omega_{A_{0}}$ in $H_{\mathrm{dR}}^{1}\left(A_{0} / F\right)$, and we choose $\eta_{A_{0}}$ so that $\left\langle\omega_{A_{0}}, \eta_{A_{0}}\right\rangle_{A_{0}}=1$ and $\left\{\omega_{A_{0}}, \eta_{A_{0}}\right\}$ is a $F$-basis of $e H_{\mathrm{dR}}^{1}\left(A_{0} / F\right)$; this is possible because the Hodge exact sequence

$$
0 \longrightarrow \Omega_{A_{0} / F}^{1} \longrightarrow H_{\mathrm{dR}}^{1}\left(A_{0} / F\right) \longrightarrow H^{1}\left(A_{0}, \mathcal{O}_{A_{0}}\right) \longrightarrow 0
$$

splits, since $A_{0}$ has CM. This yields a basis for $\operatorname{Sym}^{n} e H_{\mathrm{dR}}^{1}\left(A_{0} / F\right)$ given by the elements $\omega_{A_{0}}^{j} \otimes \eta_{A_{0}}^{n-j}$ for $j$ an integer such that $0 \leq j \leq n$.

Let $f$ be a newform of level $\Gamma_{1}\left(N^{+}\right) \cap \Gamma_{0}\left(N^{-} p\right)$ and weight $k$ defined over $F$. Let $v$ be the prime of $F$ determined by the embedding $F \subseteq \overline{\mathbb{Q}} \hookrightarrow \overline{\mathbb{Q}}_{p}$; we assume that $F_{v} / \mathbb{Q}_{p}$ is unramified and contains $\mathbb{Q}_{p^{2}}$. The Jacquet-Langlands correspondence associates to $f$ 
an algebraic modular form $f^{\mathrm{JL}}$ in $S_{k}^{\mathrm{alg}}\left(\Gamma, F_{v}\right)$, and therefore, using (39), a rigid analytic modular form $f_{\text {rig }}^{\mathrm{JL}} \in S_{k}^{\mathrm{rig}}\left(\Gamma_{p}, F_{v}\right)$. Let $\omega_{f}$ be differential associated with the rigid analytic modular form $f_{\text {rig. }}^{\mathrm{JL}}$. Recall now the definition of the function $G_{j}$ in (42), and for integers $j=n / 2, \ldots, n$ define the function

$$
H_{j}(z)=\left\langle F_{f}(z), \omega_{\mathrm{can}}^{j}(z) \eta_{\mathrm{can}}^{n-j}(z)\right\rangle .
$$

So we have $G_{j}(z)=H_{j}(z) \otimes \omega_{\text {can }}^{n-2 j}$.

Theorem 7.1 For each $j=n / 2, \ldots, n$ we have

$$
d_{\varphi}^{j} \cdot H_{j}\left(z_{A}\right)=\operatorname{AJ}\left(\Delta_{\varphi}\right)\left(\omega_{f} \otimes \omega_{A_{0}}^{j} \eta_{A_{0}}^{n-j}\right) .
$$

Proof The universal differential $\omega_{\text {can }}$ defines a differential form $\omega_{A}$ in $H_{\mathrm{dR}}^{1}(A / F)$, and choose as above $\eta_{A}$ in $H_{\mathrm{dR}}^{1}(A / F)$ in such a way that $\left\langle\omega_{A}, \eta_{A}\right\rangle_{A}=1$ and $\left\{\omega_{A}, \eta_{A}\right\}$ is a basis of $H_{\mathrm{dR}}^{1}(A / F)$. It follows from [30, Theorem 5.5] that

$$
\mathrm{AJ}\left(\Delta_{\varphi}\right)\left(\omega_{f} \otimes \omega_{A_{0}}^{j} \eta_{A_{0}}^{n-j}\right)=\left\langle\varphi^{*}\left(F_{f}\left(z_{A}\right)\right), \omega_{A_{0}}^{j} \eta_{A_{0}}^{n-j}\right\rangle_{A_{0}} .
$$

Since $\varphi^{*}\left(\omega_{A}\right)=\omega_{A_{0}}$ and $\left\langle\varphi^{*}\left(\omega_{A}\right), \varphi^{*}\left(\eta_{A}\right)\right\rangle_{A}=d_{\varphi}$, we have $\varphi^{*}\left(\eta_{A}\right)=d_{\varphi} \eta_{A_{0}}$, so we deduce

$$
\begin{aligned}
\left\langle\varphi^{*}\left(F_{f}\left(z_{A}\right)\right), \omega_{A_{0}}^{j} \eta_{A_{0}}^{n-j}\right\rangle_{A_{0}} & =d_{\varphi}^{j-n} \cdot\left\langle\varphi^{*}\left(F_{f}\left(z_{A}\right)\right), \varphi^{*}\left(\omega_{A}^{j} \eta_{A}^{n-j}\right\rangle_{A_{0}}\right. \\
& =d_{\varphi}^{j} \cdot\left\langle F_{f}\left(z_{A}\right), \omega_{A}^{j} \eta_{A}^{n-j}\right\rangle_{A}
\end{aligned}
$$

and the last expression is equal to $d_{\varphi}^{j} \cdot H_{j}\left(z_{A}\right)$.

Corollary 7.2 For each $j=n / 2, \ldots, n$ we have

$$
\frac{\delta_{p}^{n-j}\left(H_{n}\right)\left(z_{A}\right)}{t_{p}^{n-j}}=\frac{n !}{j ! \cdot d_{\varphi}^{j}} \mathrm{AJ}\left(\Delta_{\varphi}\right)\left(\omega_{f} \wedge \omega_{A_{0}}^{j} \eta_{A_{0}}^{n-j}\right) .
$$

Proof From the proof of Theorem 6.1 we see that $\Theta_{p}\left(G_{j}(z)\right)=j G_{j-1}(z)$, and therefore we have $j ! \Theta_{p}^{n-j}\left(G_{n}(z)\right)=n ! G_{j}(z)$. So using (35) we conclude $j ! \delta_{p}^{n-j}\left(H_{n}(z)\right)=n ! t_{p}^{n-j} H_{j}(z)$. The result follows from Theorem 7.1.

Acknowledgements The author thanks the referee for carefully reading the manuscript and for giving valuable suggestions which helped improving the quality of the paper. The author is supported by PRIN 2017, INdAMGNSAGA.

Open Access This article is licensed under a Creative Commons Attribution 4.0 International License, which permits use, sharing, adaptation, distribution and reproduction in any medium or format, as long as you give appropriate credit to the original author(s) and the source, provide a link to the Creative Commons licence, and indicate if changes were made. The images or other third party material in this article are included in the article's Creative Commons licence, unless indicated otherwise in a credit line to the material. If material is not included in the article's Creative Commons licence and your intended use is not permitted by statutory regulation or exceeds the permitted use, you will need to obtain permission directly from the copyright holder. To view a copy of this licence, visit http://creativecommons.org/licenses/by/4.0/.

\section{References}

1. Fabrizio Andreatta and Adrian Iovita, Katz type $p$-adic $l$-function for primes $p$ non split in the $\mathrm{cm}$ field, preprint (2019). 
2. Pierre Berthelot, Lawrence Breen, and William Messing, Théorie de Dieudonné cristalline. II, Lecture Notes in Mathematics, vol. 930, Springer-Verlag, Berlin, 1982.

3. J.-F. Boutot and H. Carayol, Uniformisation $p$-adique des courbes de Shimura: les théorèmes de čerednik et de Drinfel' d, Astérisque (1991), no. 196-197, 7, 45-158 (1992), Courbes modulaires et courbes de Shimura (Orsay, 1987/1988).

4. Massimo Bertolini, Henri Darmon, and Kartik Prasanna, Generalized Heegner cycles and $p$-adic Rankin $L$-series, Duke Math. J. 162 (2013), no. 6, 1033-1148, With an appendix by Brian Conrad.

5. Siegfried Bosch and Ulrich Görtz, Coherent modules and their descent on relative rigid spaces, J. Reine Angew. Math. 495 (1998), 119-134.

6. Pierre Berthelot and Arthur Ogus, Notes on crystalline cohomology, Princeton University Press, Princeton, N.J.; University of Tokyo Press, Tokyo, 1978.

7. P. Berthelot and A. Ogus, F-isocrystals and de Rham cohomology. I, Invent. Math. 72 (1983), no. 2, 159-199.

8. Siegfried Bosch, Lectures on formal and rigid geometry, , vol. 2105, Springer, Cham, 2014.

9. Kevin Buzzard, Integral models of certain Shimura curves, Duke Math. J. 87 (1997), no. 3, 591-612.

10. Antoine Chambert-Loir, Cohomologie cristalline: un survol, Exposition. Math. 16 (1998), no. 4, 333-382.

11. Henri Darmon, Rational points on modular elliptic curves, CBMS Regional Conference Series in Mathematics, vol. 101, Published for the Conference Board of the Mathematical Sciences, Washington, DC; by the American Mathematical Society, Providence, RI, 2004.

12. A. J. de Jong, Barsotti-Tate groups and crystals, Proceedings of the International Congress of Mathematicians, Vol. II (Berlin, 1998), no. Extra Vol. II, 1998, pp. 259-265.

13. V. G. Drinfel'd, Coverings of p-adic symmetric domains, Funkcional. Anal. i Priložen. 10 (1976), no. 2, 29-40.

14. Gerd Faltings, Crystalline cohomology of semistable curve-the $\mathbf{Q}_{p}$-theory, J. Algebraic Geom. 6 (1997), no. $1,1-18$.

15. Cameron Franc, Nearly rigid analytic modular forms and their values at CM points, ProQuest LLC, Ann Arbor, MI, 2011, Thesis (Ph.D.)-McGill University (Canada).

16. Alexandre Grothendieck, Groupes de Barsotti-Tate et cristaux de Dieudonné, Les Presses de l'Université de Montréal, Montreal, Que., 1974, Séminaire de Mathématiques Supérieures, No. 45 (Été, 1970).

17. Michael Harris, Special values of zeta functions attached to Siegel modular forms, Ann. Sci. École Norm. Sup. (4) 14 (1981), no. 1, 77-120.

18. Ki-ichiro Hashimoto, Explicit form of quaternion modular embeddings, Osaka J. Math. 32 (1995), no. 3, 533-546.

19. Ernest Hunter Brooks, Shimura curves and special values of $p$-adic $L$-functions, Int. Math. Res. Not. IMRN (2015), no. 12, 4177-4241.

20. Haruzo Hida, Elementary theory of $L$-functions and Eisenstein series, London Mathematical Society Student Texts, vol. 26, Cambridge University Press, Cambridge, 1993.

21. Luc Illusie, Cohomologie cristalline (d'après P. Berthelot), 53-60. Lecture Notes in Math., Vol. 514.

22. Adrian Iovita and Michael Spiess, Logarithmic differential forms on $p$-adic symmetric spaces, Duke Math. J. 110 (2001), no. 2, 253-278.

23. Adrian Iovita and Michael Spieß, Derivatives of $p$-adic $L$-functions, Heegner cycles and monodromy modules attached to modular forms, Invent. Math. 154 (2003), no. 2, 333-384.

24. Bruce W. Jordan and Ron A. Livné, Local Diophantine properties of Shimura curves, Math. Ann. 270 (1985), no. 2, 235-248.

25. Nicholas M. Katz, On the differential equations satisfied by period matrices, Inst. Hautes Études Sci. Publ. Math. (1968), no. 35, 223-258.

26. Nicholas M. Katz, $p$-adic $L$-functions for CM fields, Invent. Math. 49 (1978), no. 3, 199-297.

27. Nicholas M. Katz and Tadao Oda, On the differentiation of de Rham cohomology classes with respect to parameters, J. Math. Kyoto Univ. 8 (1968), 199-213.

28. Daniel Kriz, A new $p$-adic Maass-Shimura operator and supersingular Rankin-Selberg $p$-adic $l$-functions, preprint available arxiv:1805.03605.pdf (2018).

29. Matteo Longo and Maria Rosaria Pati, Exceptional zero formulae for anticyclotomic $p$-adic $L$-functions of elliptic curves in the ramified case, J. Number Theory 190 (2018), 187-211.

30. Matteo Longo and Maria Rosaria Pati, Generalized Heegner cycles on Mumford curves, Math. Z. 297 (2021), no. 1-2, 483-515.

31. Matteo Longo and Stefano Vigni, On the vanishing of Selmer groups for elliptic curves over ring class fields, J. Number Theory 130 (2010), no. 1, 128-163.

32. Matteo Longo and Stefano Vigni, A refined Beilinson-Bloch conjecture for motives of modular forms, Trans. Amer. Math. Soc. 369 (2017), no. 10, 7301-7342. 
33. Matteo Longo and Stefano Vigni, Kolyvagin systems and Iwasawa theory of generalized Heegner cycles, Kyoto J. Math. 59 (2019), no. 3, 717-746.

34. Marc Masdeu, CM cycles on Shimura curves, and p-adic L-functions, Compos. Math. 148 (2012), no. 4, 1003-1032.

35. William Messing, The crystals associated to Barsotti-Tate groups: with applications to abelian schemes, , Vol. 264, Springer-Verlag, Berlin-New York, 1972.

36. B. Mazur and William Messing, Universal extensions and one dimensional crystalline cohomology, Lecture Notes in Mathematics, Vol. 370, Springer-Verlag, Berlin-New York, 1974.

37. Andrea Mori, Power series expansions of modular forms and their interpolation properties, Int. J. Number Theory 7 (2011), no. 2, 529-577.

38. Tadao Oda, The first de Rham cohomology group and Dieudonné modules, Ann. Sci. École Norm. Sup. (4) 2 (1969), 63-135.

39. Arthur Ogus, $F$-isocrystals and de Rham cohomology. II. Convergent isocrystals, Duke Math. J. 51 (1984), no. 4, 765-850.

40. Peter Schneider, The cohomology of local systems on $p$-adically uniformized varieties, Math. Ann. 293 (1992), 623-650.

41. Jean-Pierre Serre, Géométrie algébrique et géométrie analytique, Ann. Inst. Fourier, Grenoble 6 (19551956), 1-42.

42. Goro Shimura, On some arithmetic properties of modular forms of one and several variables, Ann. of Math. (2) 102 (1975), no. 3, 491-515.

43. Jeremy Teitelbaum, On Drinfel'd's universal formal group over the $p$-adic upper half plane, Math. Ann. 284 (1989), no. 4, 647-674.

44. Thomas Zink, Cartiertheorie kommutativer formaler Gruppen, Teubner-Texte zur Mathematik [Teubner Texts in Mathematics], vol. 68, BSB B. G. Teubner Verlagsgesellschaft, Leipzig, 1984, With English, French and Russian summaries.

Publisher's Note Springer Nature remains neutral with regard to jurisdictional claims in published maps and institutional affiliations. 